\title{
The Top Quark as a Window to Beyond the Standard Model Physics
}

\author{
By \\ Chiu-Tien Yu \\ A Dissertation SUbmitted in PARTial FULFillment of THE \\ REQUIREMENTS FOR THE DEGREE OF \\ Doctor of Philosophy \\ (PHYSICS) \\ at the \\ UNIVERSITY OF WISCONSIN - MADISON
}

2013

Date of final oral examination: May 2, 2013

The dissertation is approved by the following members of the Final Oral Committee:

Professor V. Barger, Professor, Physics

Professor Y. Bai, Assistant Professor, Physics

Professor S. Dasu, Professor, Physics

Professor L. Everett, Associate Professor, Physics

Dr. R. Harnik, Scientist, Fermilab 


\section{Abstract}

The top quark was the last of the Standard Model quarks to be discovered, and is of considerable interest. The closeness of the top quark mass to the electroweak scale is suggestive that the top quark could be closely related to the mechanisms for electroweak symmetry breaking. Any new physics in electroweak symmetry breaking models could then preferentially couple to the top quark, making the top quark a promising probe for new physics. In this thesis, we will explore two aspects of the top quark as a harbinger to new physics: the top forward-backward asymmetry as seen at the Tevatron and the search for stops. In this thesis, we will discuss the Asymmetric Left-Right Model (ALRM), a model that is based on the gauge group $U^{\prime}(1) \times S U(2) \times S U^{\prime}(2)$ with couplings $g_{1}^{\prime}, g_{2}^{\prime}$, and $g^{\prime}$ associated with the fields $B^{\prime}, W, W^{\prime}$, respectively, and show how this model can explain the top forwardbackward asymmetry. We will then explore the scalar sector of the ALRM, and provide a specific Higgs mechanism that provides the masses for the $W^{\prime}$ and $Z^{\prime}$ bosons. The top forward-backward asymmetry is a test of invariance of chargeconjugation. Thus, we look at the $X$-gluon model, a model that was motivated by the top forward-backward asymmetry, and show that one can look at the longitudinal polarization of the top-quark to test parity conservation. Finally, we investigate searches for stop squarks, the supersymmetric partner of the top quark, at the Large Hadron Collider (LHC) using shape-based analyses. 


\section{Acknowledgements}

First and foremost, I would like to thank my Ph.D. advisor, Vernon Barger, for his advice, valuable insights, and unwavering support in all my endeavors. I thank the current and former members of the Pheno group at Wisconsin for getting me started in the field.

I am also grateful to the theory group at Fermilab for their hospitality in my last two years of graduate study; in particular, I would like to thank my fellowship supervisor Roni Harnik and my collaborators: Patrick Fox, Joseph Lykken, Matthew Buckley, Daniele Alves, Wolfgang Altmannshofer, and Felix Yu.

My life in Madison would not have been as colorful were it not for the Brunch Bunch, and my apartment-mates.

Finally, I am most thankful for my parents for their unconditional love and support. 


\section{List of Figures}

$168 \%$ and $95 \%$ confidence level contours of the $W$ boson mass, $M_{W}$, and top quark mass, $m_{t}$. The blue allowed regions are the results of the fit including the Higgs mass, $m_{h}$, measurements while the grey regions are the results of the fit excluding the $m_{h}$ measurements. [1] . 4

2 One of the main contributions to the quadratic divergences in the Higgs mass is from the top quark, $t$, loop. This contribution can be cancelled by a corresponding stop, $\tilde{t}$, loop. . . . . . . . . . . . 5

3 Feynman diagrams showing the SM contributions to $A_{F B}$. A small, non-zero $A_{F B}$ arises from interference between the tree-level and boxdiagrams for $q \bar{q} \rightarrow t \bar{t}$ (top two diagrams) as well as contributions from extra jet radiation (bottom two diagrams). [2] . . . . . . . . . . . . . 7

4 Tree-level ALRM contributions to $\bar{t} t$ production. $q=d_{L, R}, u_{L}$ and $\bar{q}=\bar{d}_{L, R}, \bar{u}_{L}$. The QCD gluon-gluon fusion diagram is not shown; it is included in our calculations but it is subdominant at the Tevatron energy. . . . . . . . . . . . . . . . . . . 13

$5 \quad$ Tree-level diagrams for the $W^{\prime} W^{\prime}$ production in ALRM. $q=d_{L, R}, u_{L}$ and $\bar{q}=\bar{d}_{L, R}, \bar{u}_{L} \ldots \ldots \ldots \ldots \ldots \ldots$ 
6 Comparison of the $\Delta y=y_{t}-y_{\bar{t}}$ distribution in the $p \bar{p}$ frame of the ALRM (solid) with $M_{Z^{\prime}}=900 \mathrm{GeV}, M_{W^{\prime}}=175 \mathrm{GeV}$, and $g_{2}^{\prime}=1$ with the SM (dashed) at the Tevatron. . . . . . . . . . . . . . . . 20

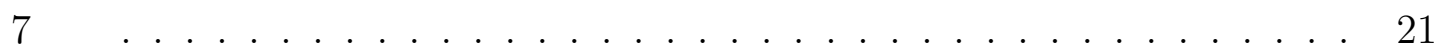

$8 \quad M_{t \bar{t}}$ distribution of CDF data [3], MadEvent SM (red solid), and ALRM (blue dashed ). $K=1.31$ has been included uniformly across all bins. . . . . . . . . . . . . . . . . . . . . . . . . 22

$9 \quad$ Cross-sections [fb] vs. $\sqrt{s}$ for various vector boson pairs in $p p$ collisions. . . . . . . . . . . . . . . . . . . . . 22

10 Dependence of $M_{W^{\prime}}$ on $g_{2}^{\prime}$ and $M_{Z^{\prime}}$. The relationship is given by Eq. $\mid 3.5$. . . . . . . . . . . . . . . . . . . . . . . . . 29

$11 d \sigma / d M_{t \bar{t}}$ distribution of CDF data(points with uncertainties) vs. ALRM (solid histograms) for the model benchmark point of $g_{2}^{\prime}=3.0, M_{W^{\prime}}=$ $700 \mathrm{GeV}$, and $M_{Z^{\prime}}=1 \mathrm{TeV}$. . . . . . . . . . . . . . . . . . 32

12 Comparison of CDF data vs. ALRM predictions in (a) $\Delta y$ distribution and (b) $A_{F B}$ in the $p \bar{p}$ CM frame vs. $M_{t \bar{t}}$ for the model benchmark point of $g_{2}^{\prime}=3, M_{W^{\prime}}=700 \mathrm{GeV}$, and $M_{Z^{\prime}}=1 \mathrm{TeV}$. The points with the uncertainties are the CDF measurements and the solid histograms are the ALRM predictions including the SM QCD contribution. . . . 34 
13 The asymmetry in the $t \bar{t}$ CM frame vs. the invariant $t \bar{t}$ mass in the ALRM for $g_{2}^{\prime}=3.0, M_{W^{\prime}}=700 \mathrm{GeV}$, and $M_{Z^{\prime}}=1 \mathrm{TeV}$. The decrease in $A_{F B}$ above $1 \mathrm{TeV}$ is due to the dominance of the $Z^{\prime}$ contribution to the cross-section. . . . . . . . . . . . . . . . . . . . . . . . . 35

14 Individual contributions to $d_{R} \bar{d}_{R} \rightarrow t \bar{t}$ at a CM scattering angle of $30^{\circ}$. We plot the magnitude of the interference term. The $Z^{\prime}$ contribution dominates the subprocess in the $1 \mathrm{TeV}$ region. The parton distributions emphasize the low $\hat{s}$ region. . . . . . . . . . . . . . . . . 36

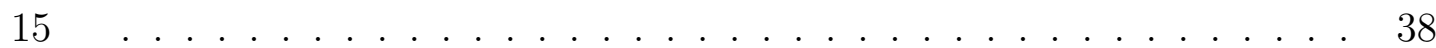

$16 \sigma(p p \rightarrow t \bar{t}+X)[\mathrm{pb}]$ vs. $\sqrt{s}[\mathrm{TeV}]$ for the SM and ALRM at the LHC. Also plotted is the new physics cross-section for $p p \rightarrow t W^{\prime}+X$. . . . 39

17 Feynman diagram of the $s$-channel exchanges of the gluon and $X$ gluon in the $q \bar{q} \rightarrow t \bar{t}$ subprocess and the definitions of $\theta$ and $\psi$. The dotted line for $t$ denotes a boost into the $t$ rest frame. . . . . . . . . . . 45

18 The new physics (NP) contributions to $d \sigma / d M_{t \bar{t}}$ vs. $M_{t \bar{t}}$ in the $X-$ gluon model at the Tevatron. . . . . . . . . . . . . . . . . . . . . . . 49

$19 \quad A_{F B}$ and $P_{\|}$vs. $M_{t \bar{t}}$ for $M_{X}=420 \mathrm{GeV}, \Gamma_{X}=42 \mathrm{GeV}$, and $\left|g_{A}\right|=g_{s} / 3.50$

20 Stop pair production at $\sqrt{s}=8 \mathrm{TeV}$, calculated at NLO using Prospino [4]. 
21 Left: Differential distribution of events for $20 \mathrm{fb}^{-1}$ with respect to $\mathbb{E}_{T}$ of QCD (blue) and $t \bar{t}$ (green), and the total background (black) passing the hadronic trigger. The analytic fits to Eq. (5.4) using the parameters in Table 9 are shown in red for QCD (dashed), $t \bar{t}$ (dotted) and their sum (solid). Right: Differential distribution of events corresponding to $20 \mathrm{fb}^{-1}$ with respect to $\mathbb{E}_{T}$ for signal $\tilde{t} \overline{\tilde{t}} \rightarrow t \bar{t} \chi \chi$ passing the hadronic trigger for a range of stop and LSP masses $\left(m_{\tilde{t}}, m_{\chi}\right) . \quad$. 66

22 Differential distribution of $t \bar{t}$ events with respect to $M_{T}^{W}$ (black). The analytic fit (Eq. (5.4) using the parameters of Table 10) is shown in red. Also shown are the differential distributions of stop signal events with respect to $M_{T}^{W}$ for a range of stop and LSP masses. The semileptonic event selection is described in Section $\mid 5.4\rceil$. . . . . . . . . . 68

23 Left: Signal trigger efficiency as a function of stop and LSP masses for hadronic event selection. Right: Signal cross section times trigger efficiencies as a function of stop and LSP masses. Like all such plots in this paper, the contours are extrapolated from a grid of Monte Carlo results with $5-25 \mathrm{GeV}$ spacing in $m_{\tilde{t}}$ and $m_{\chi}$. The degeneracy line $\left(m_{\tilde{t}}=m_{t}+m \chi\right)$ is shown in black. . . . . . . . . . . . . . . . 70 
24 Expected sensitivity, in standard deviations, for the hadronic $\mathbb{E}_{T}$ shape analysis as a function of the stop and LSP masses. The test statistic is computed from 200 pseudo-experiments of $20 \mathrm{fb}^{-1}$. In the left-hand plot the uncertainty on the background $\mathbb{E}_{T}$ shape are as shown in Table 9 and in the right-hand plot these errors have been inflated by a factor of 3. . . . . . . . . . . . . . . . . . . . . 84

25 Left: $S / \sqrt{O}$ for $100-200 \mathrm{GeV}$ region of signal plus background $\mathbb{E}_{T}$ distribution. Right: $S / \sqrt{O}$ for $200-400 \mathrm{GeV}$ region. These are computed with an unrealistic assumption of no systematics. . . . . . 85

26 The median expected exclusion, for background only pseudo experiments, on a stop-neutralino simplified model whose masses are related by $m_{\tilde{t}}-m_{\chi}=m_{t}$. . . . . . . . . . . . . . . . . . . . . . . . . . . 85

27 Left: Semi-leptonic trigger efficiency for semi-leptonic events as a function of stop and LSP masses. Right: Cross section times efficiency for the semi-leptonic selection criteria as a function of stop and LSP masses. ....................... . . . 86

28 Expected number of standard deviations that the supersymmetric stop signal can be excluded by using 200 pseudo-experiments of $20 \mathrm{fb}^{-1}$, applying the $M_{T}^{W}$ shape analysis. In the left-hand plot the uncertainty on the background $\mathbb{E}_{T}$ shape are as shown in Table $\mid 10$ and in the righthand plot these errors have been inflated by a factor of 3. 
29 Left: $S / \sqrt{O}$ for $150-250 \mathrm{GeV}$ region of signal plus background $M_{T}^{W}$ distribution. Right: $S / \sqrt{O}$ for $250-400 \mathrm{GeV}$ region. These are computed with an unrealistic assumption of no systematics. . . . . . 88

30 Expected sensitivity, in standard deviations, to SUSY stop signals using a combination of $\mathbb{E}_{T}$ and $M_{T}^{W}$ shape analyses, included all fitting errors in the maximum likelihood method. . . . . . . . . . . . . . . . 89 


\section{List of Tables}

1 Couplings of the photon, the Z-boson, and the Z'-boson in the Asymmetric Left-Right Model. The second row in the table outlines the generic couplings which applies to various particles as listed in the subsequent rows. We define the coupling strength $g_{Z}=e /\left(s_{W} c_{W}\right)$.

Note that $T_{3}^{\prime}=0$ for the SM left-handed doublets. . . . . . . . . . . . 13

$2 \quad$ Reduced $\chi_{\text {red }}^{2}$ values for the CDF data versus predictions from the ALRM and the SM . . . . . . . . . . . . . . . . . . . . . . . . . 18

$3 \quad$ Comparison of the effects of the value of $g_{2}^{\prime} \ldots \ldots \ldots \ldots$

4 Cross-sections $[\mathrm{pb}]$ of $q \bar{q} \rightarrow t \bar{t}$ for $\Delta y>0$ and $\Delta y<0$, where $\Delta y=$ $y_{t}-y_{\bar{t}}$ in the ALRM with $M_{Z^{\prime}}=900 \mathrm{GeV}$ and $g_{2}^{\prime}=1$. The subprocess

$\mathrm{CM}$ energy is fixed at $\sqrt{\hat{s}}=500 \mathrm{GeV}, \mu_{F}=\mu_{R}=M_{t}=173.1 \mathrm{GeV}$. The QCD contributions to $A_{F B}$ are not included. . . . . . . . . . . . 19

5 Expected values for $\sigma\left(V_{1} V_{2}\right)[\mathrm{pb}]$ at the Tevatron $(\sqrt{s}=2 \mathrm{TeV})$ and the LHC $(\sqrt{s}=14 \mathrm{TeV}) . K=1.31$ has been included...... .23 
$6 \quad$ ALRM predictions for the $t \bar{t}$ total cross-section $\sigma(t \bar{t})$, the forwardbackward asymmetry in the $p \bar{p}$ CM frame $\left(A_{F B}\right)$, and the cross-sections for the specified ranges of rapidity differences $\Delta y$ and $M_{t \bar{t}}$ invariant mass ranges. $M_{Z^{\prime}}$ is determined by Eq.3.5. A QCD correction factor $K=1.3$ is included in the cross-section calculation. The ALRM asymmetry numbers are the new physics contributions only and do not include the SM QCD contribution, so they should be compared with the final row in the table. The SM values are based on the MCFM study of Ref.[5]. The last row is the New Physics (NP) contribution inferred from the differences of data and SM entries. . . . . . . . . . . 33

$7 \quad \chi^{2} /$ d.o.f. values for various $g_{2}^{\prime}$ and $M_{W^{\prime}}$ mass values using $A_{F B}$ in the $7 M_{t \bar{t}}$ bins and the total cross-section $\sigma(t \bar{t})$. We have included a

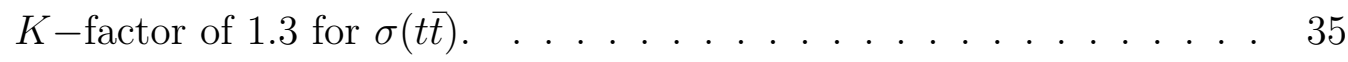

$8 \quad$ Helicity amplitudes for $q \bar{q} \rightarrow t \bar{t} \quad \ldots \ldots$. . . . . . . . . . . 46

\begin{tabular}{|ll|l|l|}
\hline 9 & Best fit parameters for QCD and $t \bar{t} \mathbb{E}_{T}$ distributions, fit to (Eq. & 5.4 ) \\
\hline
\end{tabular} for an integrated luminosity of $20 \mathrm{fb}^{-1}$. Note that these errors are correlated with each other and with the normalizations $(A, B)$, which in turn depend on the amount of integrated luminosity considered. See text for details. . . . . . . . . . . . . . . . . . . . . . . 72 
10 Best fit slope parameters for background $M_{T}^{W}$ distribution, fit to (Eq. 5.7 ). Note that the fit errors are correlated with each other and with the normalizations $(A, B)$, which in turn depend on the amount of integrated luminosity considered. . . . . . . . . . . . . . . . . 80 


\section{Contents}

Abstract i

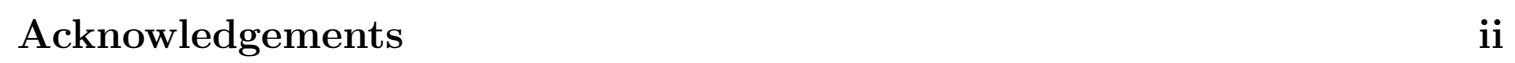

$\begin{array}{lll}1 & \text { Introduction } & 1\end{array}$

1.1 The Top Quark . . . . . . . . . . . . . . . . . 1

1.2 Top Forward-Backward Asymmetry . . . . . . . . . . . . . . . . . . . 5

2 Asymmetric Left-Right Model and the Top Pair Forward-Backward $\begin{array}{ll}\text { Asymmetry } & 9\end{array}$

2.1 Introduction . . . . . . . . . . . . . . . . . . . . . . 9

$2.2 \quad$ Asymmetric Left-Right Model . . . . . . . . . . . . . . . . . . . . . . 11

2.3 Top Pair Asymmetry and Collider Signals . . . . . . . . . . . . . . . 17

$2.4 \quad W^{\prime}$ and $Z^{\prime}$ Decays . . . . . . . . . . . . . . . . . . 23

2.5 Summary . . . . . . . . . . . . . . . . . . . 24

3 Tevatron Asymmetry of Tops in a W', Z' Model 25

3.1 Introduction . . . . . . . . . . . . . . . . . . . . . 25

3.2 Asymmetric Left-Right Model . . . . . . . . . . . . . . . . . . . . . . 27

$3.2 .1 \quad W^{\prime}, Z^{\prime}$ Mass Relation . . . . . . . . . . . . . . . . . . . . . 28

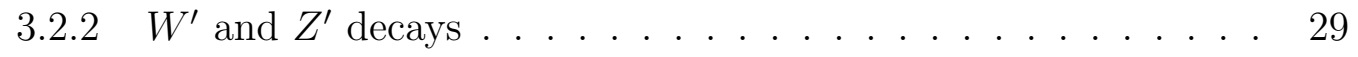


3.3 Results . . . . . . . . . . . . . . . . . . . . . . . . . . . 31

3.4 Predictions at the $\mathrm{LHC} \ldots \ldots \ldots \ldots \ldots$

3.4 .1 Z' signatures $\ldots \ldots \ldots \ldots \ldots \ldots$. . . . . . . . . . . 32

3.4 .2 W' signatures . . . . . . . . . . . . . . . . . . . . . . 37

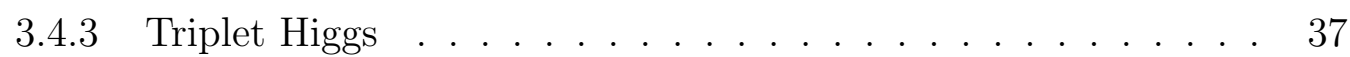

3.5 Conclusions . . . . . . . . . . . . . . . . . . . 40

4 Looking for parity nonconservation from strong interactions beyond

QCD 41

4.1 Introduction $\ldots \ldots \ldots \ldots \ldots \ldots \ldots \ldots$

$4.2 \quad X$-gluon Model $\ldots \ldots \ldots \ldots \ldots \ldots$

4.3 Phenomenology . . . . . . . . . . . . . . . . . . . . . . 48

4.4 Conclusions $\ldots \ldots \ldots \ldots$

5 Stops and MET: the shape of things to come 52

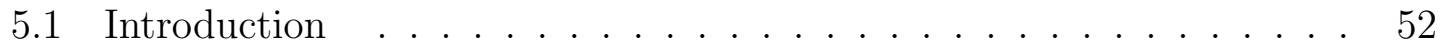

$5.2 \quad \mathbb{E}_{T}$ and $\mathbb{E}_{T}$-related Methods $\ldots \ldots \ldots \ldots \ldots$

5.3 LHC Search for Hadronic Stops $\ldots \ldots \ldots$

$5.4 \quad$ Study of $M_{T}^{W}$ shapes $\ldots \ldots \ldots \ldots \ldots \ldots$

5.5 Conclusion $\ldots \ldots \ldots \ldots \ldots \ldots \ldots \ldots \ldots$

$\begin{array}{ll}\mathrm{A} & 90\end{array}$

\begin{tabular}{ll}
\hline Bibliography & 92
\end{tabular} 


\section{Chapter 1}

\section{Introduction}

\subsection{The Top Quark}

The existence of the top quark, along with that of the bottom quark, $b$, was postulated by Makoto Kobayashi and Toshihide Maskawa in 1973 to explain the observed $\mathrm{CP}$ violations in kaon decay [6]. Their postulation was motivated by the GlashowIliopoulos-Maiani (GIM) mechanism, which suppresses dangerous flavor-changing neutral currents (FCNCs) by canceling the contributions to FCNCs between the different generations of quarks and predicted the existence of the charm quark. The discovery of the charm quark via the $J / \psi$ meson secured the GIM mechanism as a part of the SM, and gave credibility to the postulation of a third generation of quarks by Kobayashi and Maskawa. The discovery of the tau lepton in 1974 broke the symmetry in the number of lepton and quark generations, and implied the existence of a pair of third generation of quarks, further solidifying Kobayashi and Maskawa's postulate.

In 1977, the E288 experiment, led by Leon Lederman at Fermilab, discovered the bottom quark through the production of bottomonium, which strongly suggested 
the existence of the top quark to complete the pair. The top quark was expected to be heavier than the bottom, which is around $4 \mathrm{GeV}$, and therefore requiring more energy in particle collisions for discovery. However, the general expectation was that the top quark would be close to discovery. The lack of detection at the Super Proton Synchrotron (SPS) at CERN pushed the lower bound on the top mass to $41 \mathrm{GeV}$, and then to $77 \mathrm{GeV}$ when neither the Collider Detector at Fermilab (CDF) nor the group at CERN had uncovered the top quark. At this point, the experiments at CERN had reached their limit due to the beam energies; the collisions at the SPS would not have enough energy to produce top quarks heavier than $77 \mathrm{GeV}$. Instead, the search became the territory of the two experiments at the Tevatron proton-antiproton collider at Fermilab: CDF and D $\emptyset$. By 1992, the lower bound on the mass of the top quark was pushed up to $91 \mathrm{GeV}$. This proved to be a significant milestone as the top was now heavier than the $W$ boson. Before, a possible production mode for the top quark was a $W$ boson decaying into a top quark and anti-bottom quark. However, since the top mass was above the mass of the $W$ boson, this production mode was closed off and the main production mode would have to be the creation of a top-antitop pair. The top-antitop pair then decay into a pairs of $W$ boson-bottom quark and $W$ boson-antibottom quark, respectively. The lower bound of the top mass slowly crept up to $108 \mathrm{GeV}$ by CDF, and then to $131 \mathrm{GeV}$ by D $\emptyset$ [7].

The top quark was finally discovered in February 1995 by the CDF and D $\emptyset$ experiments. One of the most striking features of a top quark event is the presence of jets produced by its partner, the bottom quark. The bottom quark travels as part 
of a baryon or meson jet for roughly half a millimeter from the point of generation, after which it decays, giving the bottom-quark jet a unique signature in detectors. Both CDF and $\mathrm{D} \emptyset$ found events in which at least one of the $W$ bosons decayed into an electron or muon plus a neutrino, and a significant number of those events contained evidence for one or two bottom quarks. The combination of these two signatures suggested that what the two experiments were seeing was indeed the production of top-antitop pairs, with a top quark mass of $178 \pm 8 \pm 10 \mathrm{GeV}$ for CDF [8] and $199 \pm 20 \pm 22 \mathrm{GeV}$ for $\mathrm{D} \emptyset[9]$

After the initial discovery, the focus of the two experiments turned to measuring the properties of the newly discovered quark. The top quark is unique amongst the quarks; it is about 43 times heavier than the next heaviest quark, the bottom, at $173.5 \pm 0.6 \pm 0.8 \mathrm{GeV}[10]$. Furthermore, the top quark has a lifetime of around $5 \times 10^{-25}$ seconds [11], which is about twenty times shorter than the timescale for strong interactions of about $3 \times 10^{-24}$ seconds. Thus, the top quark decays before it hadronizes, unlike all the other quarks. As a result, the decay products of the top quark carry unique information about the top, making it possible to study the properties of the "bare" top quark.

In addition, the top quark mass plays an important role in precision electroweak measurements. Both the top quark and Higgs boson come into play in the one-loop corrections to the $W$ and $Z$ boson masses. Thus, knowledge of the the top quark mass, in addition to the $W$ and $Z$ boson masses, allows for an accurate prediction of the Higgs boson mass, as seen in Fig. 1. This procedure has been done by several 
groups, including GFitter [12].

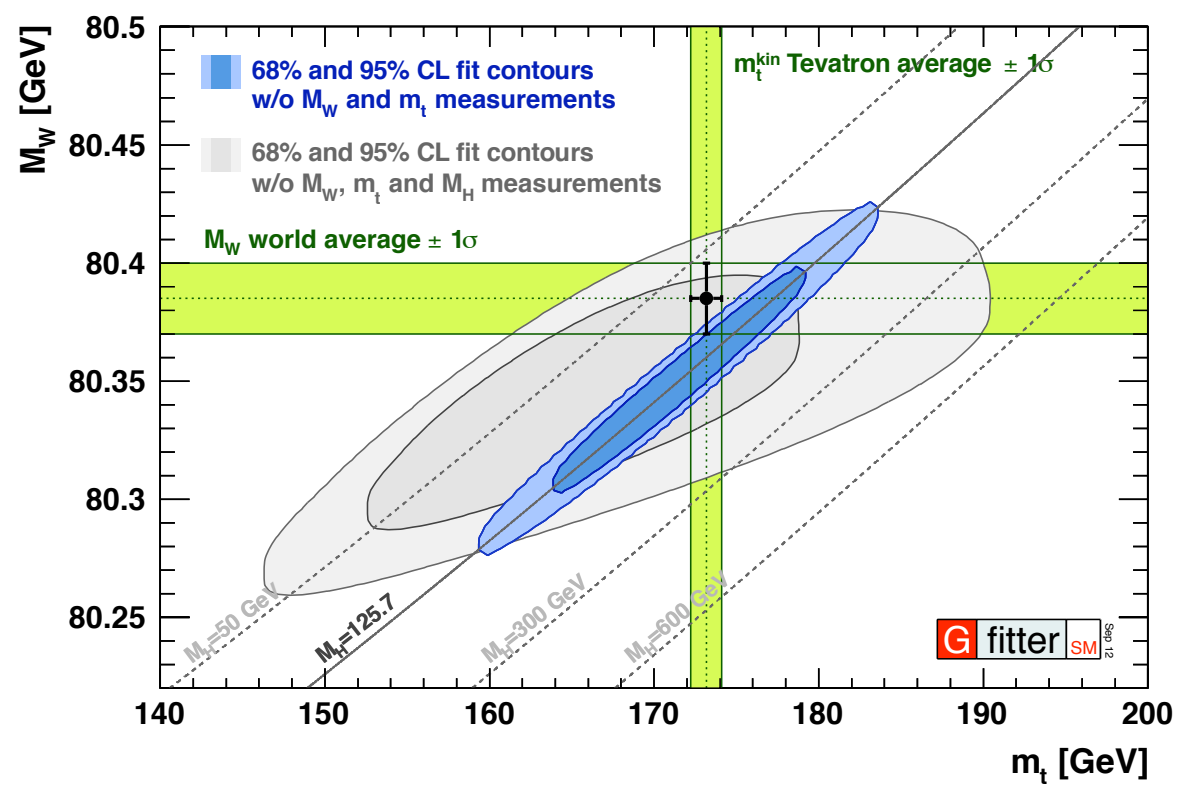

Figure 1: $68 \%$ and $95 \%$ confidence level contours of the $W$ boson mass, $M_{W}$, and top quark mass, $m_{t}$. The blue allowed regions are the results of the fit including the Higgs mass, $m_{h}$, measurements while the grey regions are the results of the fit excluding the $m_{h}$ measurements. [1]

The high mass of the top quark means that, out of all the SM fermions, the Higgs boson couples most strongly to the top quark. Thus, in addition to providing constraints on the Higgs mass, the top quark could provide much insight into the scalar sector of the SM. For example, the one-loop contributions to the Higgs mass contain quadratic divergences, with one of the largest contributions coming from the top-quark. This is known as the Higgs naturalness problem. In order to avoid a large degree of fine-tuning, one expects the existence of a top partner to cancel the 
contributions to the quadratic divergences of the Higgs mass from the top quark (Fig. 2). In supersymmetry, the top partner is the squark called the stop, $\tilde{t}$.

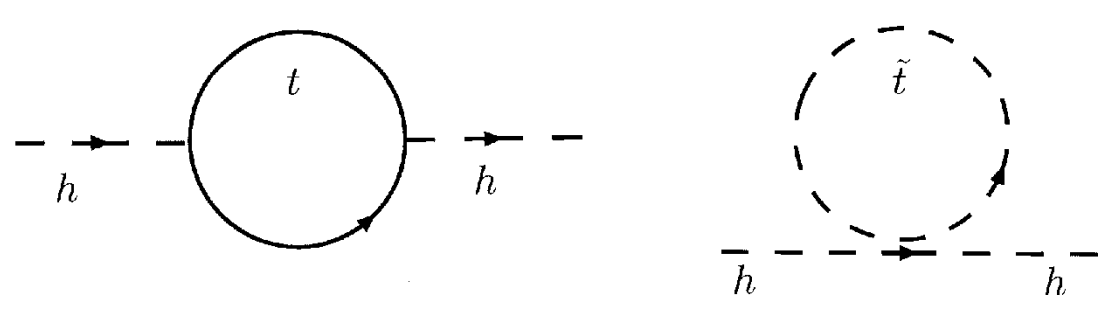

Figure 2: One of the main contributions to the quadratic divergences in the Higgs mass is from the top quark, $t$, loop. This contribution can be cancelled by a corresponding stop, $\tilde{t}$, loop.

The closeness of the top quark mass to the electroweak scale is suggestive that the top quark could be closely related to the mechanisms for electroweak symmetry breaking. Any new physics in electroweak symmetry breaking models could then preferentially couple to the top quark, making the top quark a promising probe for new physics.

\subsection{Top Forward-Backward Asymmetry}

The top forward-backward asymmetry is one such probe for new physics. At treelevel in the Standard Model (SM), the differential cross-section for $q \bar{q} \rightarrow t \bar{t}$, where $q=u, d$, is invariant under the interchange of the $t$ and $\bar{t}$ momenta. However, interference between tree-level and one-loop diagrams for $q \bar{q} \rightarrow t \bar{t}$ give rise to a small asymmetry (Fig. 3). At the Tevatron, the observable used to measure this asymmetry 
is

$$
A_{F B}=\frac{N(\Delta y>0)-N(\Delta y<0)}{N(\Delta y>0)+N(\Delta y<0)}
$$

where $\Delta y=y_{t}-y_{\bar{t}}$ is the difference in rapidities of the top quark and antiquark, with the $z$-axis defined as the direction of the proton. Since the Tevatron is a $p \bar{p}$ collider, the directions of the initial quark and antiquark are known; the initial quark is coming from the proton while the initial antiquark is coming from the antiproton. Thus, the asymmetry in rapidity difference is equivalent to an asymmetry in the polar angle $(\theta)$ between the momentum of the top quark and the direction of the initial quark in the center of mass frame,

$$
A_{F B}=\frac{N(\cos \theta>0)-N(\cos \theta<0)}{N(\cos \theta>0)+N(\cos \theta<0)} .
$$

Both the CDF and D $\varnothing$ experiments at the Tevatron have consistently measured anomalously large values of $A_{F B}$ [13] [14], which has generated much excitement. In particular, this anomaly has not disappeared with increased luminosity. Thus, it is worth pursuing the models that have been proposed to explain the anomalous measurement.

In this thesis, we will explore two aspects of the top quark as a harbinger to new physics: the top forward-backward asymmetry as seen at the Tevatron and the search for stops. In Chapter 2, we will discuss the Asymmetric Left-Right Model (ALRM), a model that is based on the gauge group $U^{\prime}(1) \times S U(2) \times S U^{\prime}(2)$ with couplings $g_{1}^{\prime}, g_{2}^{\prime}$, and $g^{\prime}$ associated with the fields $B^{\prime}, W, W^{\prime}$, respectively, and show how this model 

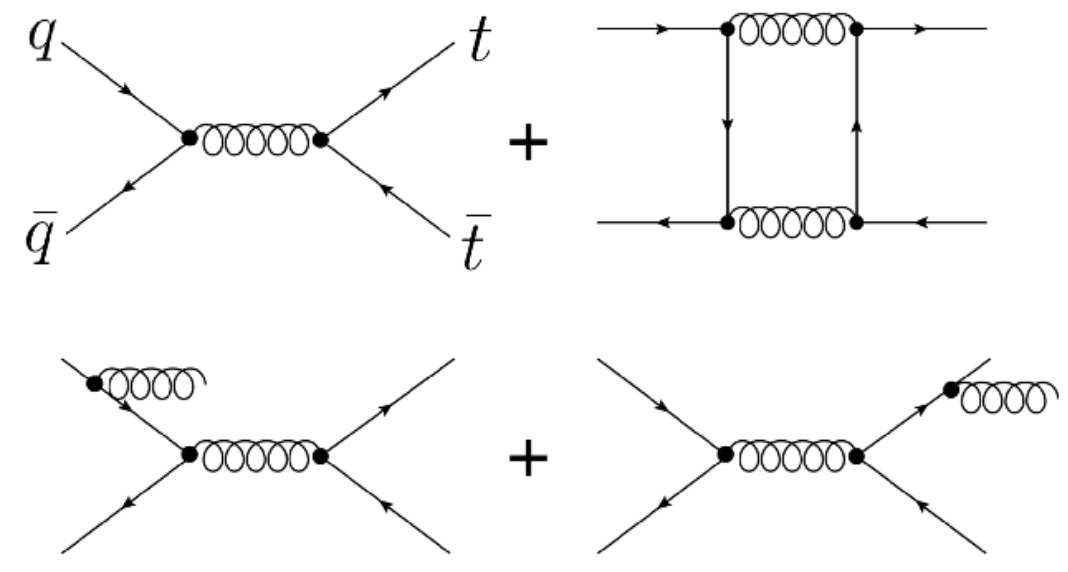

Figure 3: Feynman diagrams showing the SM contributions to $A_{F B}$. A small, nonzero $A_{F B}$ arises from interference between the tree-level and box-diagrams for $q \bar{q} \rightarrow t \bar{t}$ (top two diagrams) as well as contributions from extra jet radiation (bottom two diagrams). [2] 
can explain the top forward-backward asymmetry. In Chapter 3, we will discuss the scalar sector of the ALRM, and provide a specific Higgs mechanism that provides the masses for the $W^{\prime}$ and $Z^{\prime}$ bosons. The top forward-backward asymmetry is a test of invariance of charge-conjugation. In Chapter 4, we look at the $X$-gluon model, a model that was motivated by the top forward-backward asymmetry, and show that one can look at the longitudinal polarization of the top-quark to test parity conservation. Finally, in Chapter 5, we explore searches for stop squarks at the Large Hadron Collider (LHC) using shape-based analyses. 


\section{Chapter 2}

\section{Asymmetric Left-Right Model and the Top Pair Forward-Backward}

\section{Asymmetry}

\subsection{Introduction}

The top quark is the only fermion whose mass is close to the scale of electroweak symmetry breaking. As such, the top quark is of particular interest in particle physics for it provides a window to many new physics models. In 2009, both the CDF and D $\phi$ experiments at the Tevatron have observed a sizeable forward-backward asymmetry in top anti-top pair events in which one top decays semi-leptonically. The recent CDF measurement of the top asymmetry in the $p \bar{p}$ frame, based on $3.2 \mathrm{fb}^{-1}$ of data, is $A_{F B}^{p \bar{p}}=0.19 \pm 0.07_{\text {stat. }} \pm 0.02_{\text {syst. }}$ [15], while the next-to-leading-order (NLO) SM prediction [16] [17] [18] is $A_{F B}^{p \bar{p}(S M)} \simeq 0.080$, with a factorization=renormalization scale uncertainty 0.007 [16], but the true theoretical uncertainty may be larger. The discrepancy between the measurement and prediction of the $A_{F B}^{p \bar{p}}$ is a possible indication 
of new physics.

Several models have been proposed to explain the $A_{F B}^{p \bar{p}}$ anomaly. The models are subject to three constraints: $\sigma(t \bar{t}), d \sigma / d M_{t \bar{t}}$, and $A_{F B}^{p \bar{p}}$. The measured $t \bar{t}$ cross section by CDF of $\sigma(t \bar{t})=8.1_{-0.87}^{+0.98} \mathrm{pb}$ for $m_{t}=173.1 \mathrm{GeV}$ [19] is in good agreement with the SM prediction of $\sigma(t \bar{t})^{S M}=7.4 \pm 0.57 \mathrm{pb}$ for $m_{t}=173 \mathrm{GeV}$ as recently calculated by [20, 21] at the next-next-leading-order (NNLO) and by others [22, 23] in earlier studies. The invariant mass $M_{t \bar{t}}$ distribution is also in reasonable accord with the SM predictions. In addition to creating the appropriate $A_{F B}^{p \bar{p}}$, any new physics model must be consistent with the cross-section and $t \bar{t}$ mass distribution.

The models proposed to explain the large asymmetry can be placed into two categories. The first consists of models involving the s-channel exchange of new vector bosons with chiral couplings to the light quarks and the top quark. The most basic requirement for such a model is a spin-one, color-octet particle with nonzero axial-couplings. Ref. [24] provides limits on the axigluon mass while the authors of Ref. 25] consider a chiral color model that involves an axigluon based on the gauge group $S U(3)_{A} \times S U(3)_{B} \times S U(2)_{L} \times U(1)_{Y}$. The next category consists of models involving the t-channel exchange of particles with large flavor-violating couplings. Within this category, the models can be differentiated by the exchange of either a scalar particle $\phi$ or a vector boson. The various possibilities for a scalar particle are limited by the SM gauge structure, and therefore can be categorized by the $S U(3)_{C}$ representation of $\phi$. The authors of Ref. [26] propose the introduction of a colorsextet or a color-triplet scalar as an explanation of the top quark forward-backward 
asymmetry. The authors of Ref.[27] propose a $Z^{\prime}$ boson associated with the $U(1)_{Z^{\prime}}$ abelian gauge symmetry with a flavor off-diagonal coupling between the up and top quarks. K. Cheung et al 28] consider a $W^{\prime}$ boson with off-diagonal right-handed coupling between the down and top quarks. Other recent attempts to address the production asymmetry are found in Refs. [29, 30, 31, 32, 33, 34]

In this chapter, we discuss a model that is based on the gauge group $U^{\prime}(1) \times$ $S U(2) \times S U^{\prime}(2)$ with couplings $g_{1}^{\prime}, g_{2}^{\prime}$, and $g^{\prime}$ associated with the fields $B^{\prime}, \mathbf{W}, \mathbf{W}^{\prime}$ respectively. We introduce the model in Section II and lay out the relations between the couplings. In Section III, we estimate the model parameters and discuss potential signatures of our model at the LHC.

\subsection{Asymmetric Left-Right Model}

We begin with the gauge group $U^{\prime}(1) \times S U^{\prime}(2) \times S U(2)$. The unprimed $S U(2)$ acts on the usual SM left-handed quark doublets. The primed $S U^{\prime}(2)$ applies to the right-handed doublet $(t, d)_{R}^{T}$ in an unconventional grouping. Therefore, the model is a kind of Asymmetric Left-Right Model in which we allow different strengths of L and $\mathrm{R}$ gauge couplings.

The gauge symmetries are broken sequentially, starting with $U^{\prime}(1) \times S U^{\prime}(2) \rightarrow U_{Y}$ to obtain the SM hypercharge $\frac{Y}{2}=T_{3}^{\prime}+\frac{Y^{\prime}}{2}$, and then $U_{Y}(1) \times S U(2) \rightarrow U_{E M}$ to obtain $Q=T_{3}+\frac{Y}{2}$. By using this sequential approximation to the breaking, we preserve the SM interaction. 
After symmetry breaking, there are massive $Z^{\prime}$ and $W^{\prime \pm}$ bosons in addition to the usual weak bosons. The $W^{\prime \pm}$ have a $Z^{\prime} W^{\prime} W^{\prime}$ tri-gauge boson coupling given by $g_{2}^{\prime 2} / \sqrt{g^{\prime 2}+g_{1}^{\prime 2}}$, which is of order $\mathcal{O}(1)$. In order to preserve unitarity, the SM $Z$ also appears in the vertex $Z W^{\prime+} W^{\prime-}$ with coupling $-e \tan \theta_{W}$. Additional massive fermions will be needed for anomaly cancellation.

Our results are derived by making two successive rotations of gauge boson states. First, we make the rotation

$$
B=\left(g_{2}^{\prime} B^{\prime}+g_{1}^{\prime} W^{\prime 3}\right) / \sqrt{g_{1}^{\prime 2}+g_{2}^{\prime 2}}, Z^{\prime}=\left(-g_{1}^{\prime} B^{\prime}+g_{2}^{\prime} W^{\prime 3}\right) / \sqrt{g_{1}^{\prime 2}+g_{2}^{\prime 2}} .
$$

Then the couplings to the generators become

$$
g_{1}^{\prime} \frac{Y^{\prime}}{2} B^{\prime}+g_{2}^{\prime} T_{3}^{\prime} W_{3}^{\prime}=\left(\frac{g_{1}^{\prime} g_{2}^{\prime}}{\sqrt{g_{1}^{\prime 2}+g_{2}^{\prime 2}}}\right) \frac{Y}{2} B+\sqrt{g_{1}^{\prime 2}+g_{2}^{\prime 2}}\left(T_{3}^{\prime}-\frac{g_{1}^{\prime 2} \frac{Y}{2}}{g_{1}^{\prime 2}+g_{2}^{\prime 2}}\right) Z^{\prime} .
$$

Subsequently, we perform the usual SM rotation from the basis of $B, W^{3}$ to the basis of $A, Z$. To simplify the expressions, we denote $g^{\prime}=\sqrt{g_{2}^{\prime 2}+g_{1}^{\prime 2}}$. The SM hypercharge coupling is $g_{1}=g_{1}^{\prime} g_{2}^{\prime} / g^{\prime}=e / c_{W}$, and the SM $S U(2)$ coupling is $g_{2}=e / s_{W}$. It is also useful to note that $g_{1}^{\prime}=\left(c_{W}^{2} / e^{2}-g_{2}^{\prime-2}\right)^{-\frac{1}{2}}$.

We summarize the neutral current couplings in Table1. We use the usual notation $s_{W}=\sin \theta_{W}$ and $c_{W}=\cos \theta_{W}$ with $\theta_{W}$ the weak mixing angle. The second row in the table outlines the generic couplings that apply to various particles as listed in the subsequent rows. Note that $T_{3}^{\prime}=0$ for the SM left-handed doublets.

The spin- and color-summed amplitude squares for $q \bar{q} \rightarrow t \bar{t}$ (Fig. 4) are given by 
Table 1: Couplings of the photon, the Z-boson, and the Z'-boson in the Asymmetric Left-Right Model. The second row in the table outlines the generic couplings which applies to various particles as listed in the subsequent rows. We define the coupling strength $g_{Z}=e /\left(s_{W} c_{W}\right)$. Note that $T_{3}^{\prime}=0$ for the SM left-handed doublets.

\begin{tabular}{|c|c|c|c|}
\hline neutral boson & $\gamma$ & $Z$ & $Z^{\prime}$ \\
\hline \hline$f \bar{f}$ pair coupling & $e Q_{q}$ & $g_{Z}\left(T_{3, q}^{\mathrm{SM}}-Q_{q} s_{W}^{2}\right)$ & $g^{\prime} T_{3}^{\prime}-\frac{g_{1}^{\prime 2}}{g^{\prime}}\left(Q_{q}-T_{3, q}^{\mathrm{SM}}\right)$ \\
\hline \hline$u_{L} \bar{u}_{L}$ & $\frac{2}{3} e$ & $g_{Z}\left(\frac{1}{2}-\frac{2}{3} s_{W}^{2}\right)$ & $\frac{-g_{1}^{\prime 2}}{g^{\prime}} \frac{1}{6}$ \\
\hline$u_{R} \bar{u}_{R}$ & $\frac{2}{3} e$ & $-g_{Z}\left(\frac{2}{3} s_{W}^{2}\right)$ & $\frac{-g_{1}^{\prime 2}}{g^{\prime}} \frac{2}{3}$ \\
\hline$d_{L} \bar{d}_{L}$ & $-\frac{1}{3} e$ & $g_{Z}\left(-\frac{1}{2}+\frac{1}{3} s_{W}^{2}\right)$ & $\frac{-g_{1}^{\prime 2}}{g^{\prime}} \frac{1}{6}$ \\
\hline$d_{R} \bar{d}_{R}$ & $-\frac{1}{3} e$ & $g_{Z}\left(\frac{1}{3} s_{W}^{2}\right)$ & $-\frac{1}{2} g^{\prime}+\frac{g_{1}^{\prime 2}}{g^{\prime}} \frac{1}{3}$ \\
\hline$t_{L} \bar{t}_{L}$ & $\frac{2}{3} e$ & $g_{Z}\left(\frac{1}{2}-\frac{2}{3} s_{W}^{2}\right)$ & $\frac{-g_{1}^{\prime 2}}{g^{\prime}} \frac{1}{6}$ \\
\hline$t_{R} \bar{t}_{R}$ & $\frac{2}{3} e$ & $-g_{Z}\left(\frac{2}{3} s_{W}^{2}\right)$ & $+\frac{1}{2} g^{\prime}-\frac{g_{1}^{\prime 2}}{g^{\prime}} \frac{2}{3}$ \\
\hline$l_{L} \bar{l}_{L}$ & $-e$ & $-g_{Z}\left(\frac{1}{2}-s_{W}^{2}\right)$ & $\frac{g_{1}^{\prime 2}}{g^{\prime}} \frac{1}{2}$ \\
\hline$l_{R} \bar{l}_{R}$ & $-e$ & $g_{Z}\left(s_{W}^{2}\right)$ & $\frac{g_{1}^{\prime 2}}{g^{\prime}}$ \\
\hline$\nu_{L} \bar{\nu}_{L}$ & 0 & $g_{Z}\left(\frac{1}{2}\right)$ & $\frac{g_{1}^{\prime 2}}{g^{\prime}} \frac{1}{2}$ \\
\hline$W^{+} W^{-},(\mathrm{SM})$ & $e$ & $e \cot \theta_{W}$ & 0 \\
\hline$W^{\prime+} W^{\prime-}$ & $e$ & $-e \tan \theta_{W}$ & $g_{2}^{\prime 2} / g^{\prime}$ \\
\hline
\end{tabular}
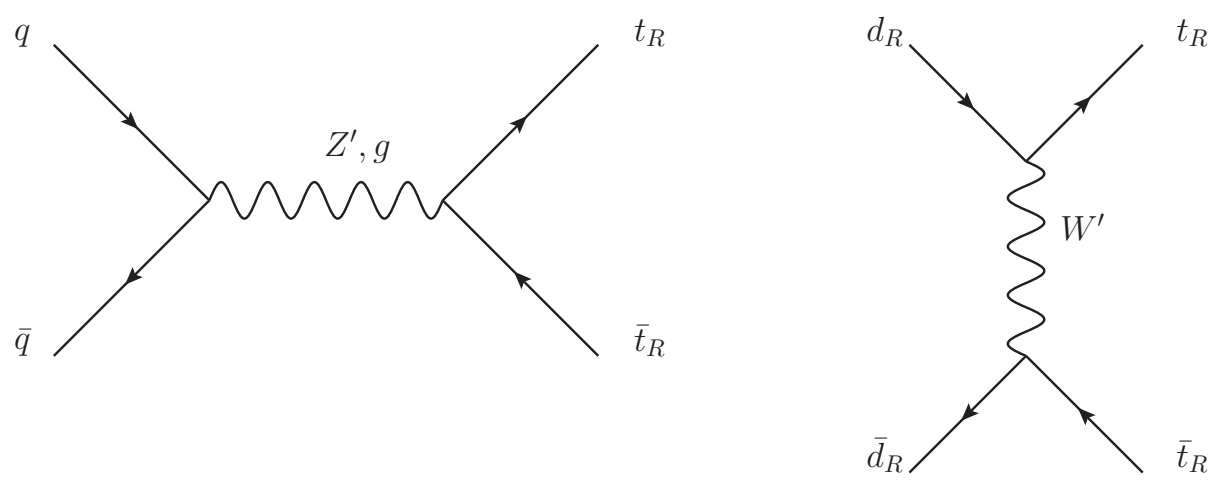

Figure 4: Tree-level ALRM contributions to $\bar{t} t$ production. $q=d_{L, R}, u_{L}$ and $\bar{q}=$ $\bar{d}_{L, R}, \bar{u}_{L}$. The QCD gluon-gluon fusion diagram is not shown; it is included in our calculations but it is subdominant at the Tevatron energy. 


$$
\begin{aligned}
& \sum|\mathcal{M}|^{2}\left(d_{R} \bar{d}_{R} \rightarrow t \bar{t}\right)= \\
&\left(\hat{u}-m_{t}^{2}\right)^{2}\left(9 \frac{g_{2}^{\prime 4}}{\left(\hat{t}-M_{W^{\prime}}^{2}\right)^{2}}+36 \frac{\left(g_{R}^{\prime d} g_{R}^{\prime \prime}\right)^{2}}{\left(\hat{s}-M_{Z^{\prime}}^{2}\right)^{2}}+12 \frac{g_{2}^{\prime 2} g_{R}^{\prime d} g_{R}^{\prime t}}{\left(\hat{t}-M_{W^{\prime}}^{2}\right)\left(\hat{s}-M_{Z^{\prime}}^{2}\right)}\right) \\
&+ 9 g_{2}^{\prime 4} \frac{m_{t}^{4}}{M_{W^{\prime}}^{4}} \frac{\left(\hat{t}-m_{t}^{2}\right)^{2}+4 M_{W^{\prime}}^{2} \hat{s}}{4\left(\hat{t}-M_{W^{\prime}}^{2}\right)^{2}}+\frac{6 g_{2}^{\prime 2} g_{R}^{\prime d} g_{R}^{\prime t} \hat{s} m_{t}^{4} / M_{W^{\prime}}^{2}}{\left(\hat{t}-M_{W^{\prime}}^{2}\right)\left(\hat{s}-M_{Z^{\prime}}^{2}\right)} \\
&+ 36\left(g_{R}^{\prime d}\right)^{2} g_{L}^{\prime \prime} \frac{g_{L}^{\prime t}\left(\hat{t}-m_{t}^{2}\right)^{2}+2 g_{R}^{\prime t} \hat{s} m_{t}^{2}}{\left(\hat{s}-M_{Z^{\prime}}^{2}\right)^{2}}+6 g_{2}^{\prime 2} g_{R}^{\prime d} g_{L}^{\prime \prime} \frac{2 \hat{s} m_{t}^{2}+\left(\hat{t}-m_{t}^{2}\right)^{2} \frac{m_{t}^{2}}{M_{W^{\prime}}^{2}}}{\left(\hat{t}-M_{W^{\prime}}^{2}\right)\left(\hat{s}-M_{Z^{\prime}}^{2}\right)} \\
&+ \frac{8 g_{2}^{\prime 2} g_{s}^{2} / \hat{s}}{\hat{t}-M_{W^{\prime}}^{2}}\left[2\left(\hat{u}-m_{t}^{2}\right)^{2}+2 \hat{s} m_{t}^{2}+\frac{m_{t}^{2}}{M_{W^{\prime}}^{2}}\left[\left(\hat{t}-m_{t}^{2}\right)^{2}+\hat{s} m_{t}^{2}\right]\right] \\
&+ \frac{16 g_{s}^{4}}{\hat{s}^{2}}\left[\left(\hat{u}-m_{t}^{2}\right)^{2}+\left(\hat{t}-m_{t}^{2}\right)^{2}+2 \hat{s} m_{t}^{2}\right] \\
& \sum|\mathcal{M}|^{2}\left(d_{L} \bar{d}_{L} \rightarrow \quad t \bar{t}\right)=\frac{36\left(g_{L}^{\prime}\right)^{2}}{\left(\hat{s}-M_{Z^{\prime}}^{2}\right)^{2}}\left[\left(g_{L}^{\prime t}\right)^{2}\left(\hat{u}-m_{t}^{2}\right)^{2}+\left(g_{R}^{\prime t}\right)^{2}\left(\hat{t}-m_{t}^{2}\right)^{2}+2 g_{L}^{\prime t} g_{R}^{\prime t} \hat{s} m_{t}^{2}\right] \\
&+\frac{16 g_{s}^{4}}{\hat{s}^{2}}\left[\left(\hat{u}-m_{t}^{2}\right)^{2}+\left(\hat{t}-m_{t}^{2}\right)^{2}+2 \hat{s} m_{t}^{2}\right]
\end{aligned}
$$

The $g_{L, R}^{\prime d}$ and $g_{L, R}^{\prime t}$ couplings are from Table I, where $g_{L, R}^{\prime d}$ is the coupling $d_{L, R} \bar{d}_{L, R} Z^{\prime}$ and $g_{L, R}^{\prime t}$ is the coupling $t_{L, R} \bar{t}_{L, R} Z^{\prime}$. Other channels can be obtained by substitutions. The $s$-channel amplitude via $Z^{\prime}$ interferes with the $t$-channel $W^{\prime}$, but not with the $s$-channel virtual gluon. The gluon fusion amplitude is the same as in the Standard Model and can be found in Ref. [35].

It is important to verify that

$$
\sum_{V^{0}=\gamma, Z, Z^{\prime}}\left(\text { coupling of } u_{L} \bar{u}_{L} \text { to } V^{0}\right) \times\left(\text { coupling of } V^{0} \text { to } W^{\prime+} W^{\prime-}\right)=0 .
$$

More explicitly,

$$
\frac{2}{3} e^{2}-\frac{e^{2}}{c_{W}^{2}}\left(\frac{1}{2}-\frac{2}{3} s_{W}^{2}\right)-\frac{g_{2}^{\prime 2} g_{1}^{\prime 2}}{g^{\prime 2}} \frac{1}{6}=0 .
$$


This guarantees acceptable high energy behavior for the subprocess $u_{L} \bar{u}_{L} \rightarrow W^{\prime+} W^{\prime-}$. Incorporating the propagators of $\gamma, Z, Z^{\prime}$, we obtain the matrix element squared

$$
\begin{aligned}
& \sum|M|^{2}\left(\bar{u}_{L} u_{L} \rightarrow W^{\prime+} W^{\prime-}\right) \\
= & {\left[\frac{2}{3} e^{2}-\frac{e^{2}}{c_{W}^{2}}\left(\frac{1}{2}-\frac{2}{3} s_{W}^{2}\right) \frac{\hat{s}}{\hat{s}-M_{Z}^{2}}-\frac{e^{2}}{6 c_{W}^{2}} \frac{\hat{s}}{\hat{s}-M_{Z^{\prime}}^{2}}\right]^{2} 4 A^{\prime}(\hat{s}, \hat{t}, \hat{u}) . }
\end{aligned}
$$

where the $s$-channel function $A^{\prime}$ is given in Eq. 2.10 below. In the $s \rightarrow \infty$ limit, $A^{\prime}(s, t, u) \longrightarrow s / M_{W^{\prime}}^{2}$, but the cancellation of couplings renders an acceptable high energy behavior. Similarly, the matrix element squared for $\bar{d}_{L} d_{L} \rightarrow W^{\prime+} W^{\prime-}$ is given by

$$
\begin{aligned}
& \sum|M|^{2}\left(\bar{d}_{L} d_{L} \rightarrow W^{\prime+} W^{\prime-}\right) \\
= & {\left[-\frac{1}{3} e^{2}-\frac{e^{2}}{c_{W}^{2}}\left(-\frac{1}{2}+\frac{1}{3} s_{W}^{2}\right) \frac{\hat{s}}{\hat{s}-M_{Z}^{2}}-\frac{e^{2}}{6 c_{W}^{2}} \frac{\hat{s}}{\hat{s}-M_{Z^{\prime}}^{2}}\right]^{2} 4 A^{\prime}(\hat{s}, \hat{t}, \hat{u}) }
\end{aligned}
$$

The differential cross-sections are given by

$$
\frac{d \sigma}{d \hat{t}}=\underbrace{\left(\frac{1}{3}\right)}_{\text {color }} \underbrace{\left(\frac{1}{8 \hat{s}}\right)}_{\text {spin-flux phase-space }} \underbrace{\left(\frac{1}{8 \pi \hat{s}}\right)} \sum|M|^{2}
$$

The charged current interaction

$$
\mathcal{L} \supset\left(g_{2}^{\prime} / \sqrt{2}\right) \bar{t}_{R} \gamma^{\mu} d_{R} W_{\mu}^{\prime}+\text { h.c. }
$$

enters the subprocess $\bar{d}_{R} d_{R} \rightarrow W^{\prime+} W^{\prime-}$. The exchange of a right-handed top in the $t$-channel for $\bar{d}_{R} d_{R} \rightarrow W^{\prime+} W^{\prime-}$ gives the necessary unitarity cancellation in the high 

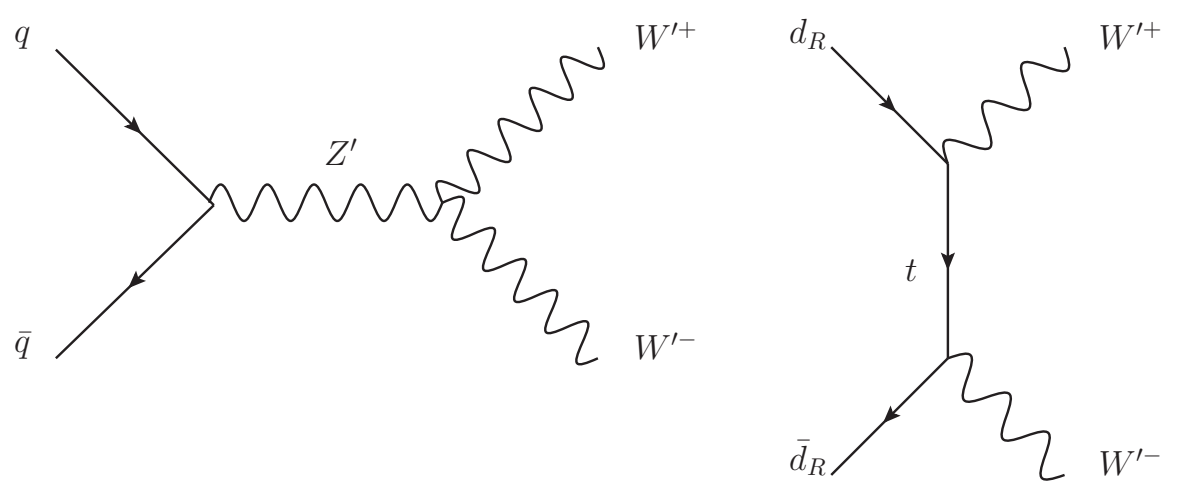

Figure 5: Tree-level diagrams for the $W^{\prime} W^{\prime}$ production in ALRM. $q=d_{L, R}, u_{L}$ and $\bar{q}=\bar{d}_{L, R}, \bar{u}_{L}$

energy limit. The matrix element squared is

$$
\begin{aligned}
& \sum|M|^{2}\left(\bar{d}_{R} d_{R} \rightarrow W^{\prime+} W^{\prime-}\right)=\left(\frac{g_{2}^{\prime}}{\sqrt{2}}\right)^{4} 4 E^{\prime}(\hat{s}, \hat{t}, \hat{u}) \\
+ & {\left[-\frac{1}{3} e^{2}-\frac{s_{W}^{2}}{3 c_{W}^{2}} e^{2} \frac{\hat{s}}{\hat{s}-M_{Z}^{2}}+\left(-\frac{g_{2}^{\prime 2}}{2}+\frac{e^{2}}{3 c_{W}^{2}}\right) \frac{\hat{s}}{\hat{s}-M_{Z^{\prime}}^{2}}\right]^{2} 4 A^{\prime}(\hat{s}, \hat{t}, \hat{u}) } \\
+ & 2\left(\frac{g_{2}^{\prime}}{\sqrt{2}}\right)^{2}\left[-\frac{1}{3} e^{2}-\frac{s_{W}^{2}}{3 c_{W}^{2}} e^{2} \frac{\hat{s}}{\hat{s}-M_{Z}^{2}}+\left(-\frac{g_{2}^{\prime 2}}{2}+\frac{e^{2}}{3 c_{W}^{2}}\right) \frac{\hat{s}}{\hat{s}-M_{Z^{\prime}}^{2}}\right] 4 I^{\prime}(\hat{s}, \hat{t}, \hat{u})
\end{aligned}
$$

The functions $A^{\prime}, I^{\prime}, E^{\prime}$ are

$$
\begin{aligned}
A^{\prime}(\hat{s}, \hat{t}, \hat{u}) & =\frac{1}{4}\left(\frac{\hat{u} \hat{t}}{M_{W^{\prime}}^{4}}-1\right)\left(1-4 \frac{M_{W^{\prime}}^{2}}{\hat{s}}+12 \frac{M_{W^{\prime}}^{4}}{\hat{s}^{2}}\right)+\frac{\hat{s}}{M_{W^{\prime}}^{2}}-4 \\
I^{\prime}(\hat{s}, \hat{t}, \hat{u}) & =\left[\frac{1}{4}\left(\frac{\hat{u} \hat{t}}{M_{W^{\prime}}^{4}}-1\right)\left(1-2 \frac{M_{W^{\prime}}^{2}}{\hat{s}}-\frac{4 M_{W^{\prime}}^{4}}{\hat{s} \hat{t}}\right)+\frac{\hat{s}}{M_{W^{\prime}}^{2}}-2+2 \frac{M_{W^{\prime}}^{2}}{\hat{t}}\right] \frac{\hat{t}}{\hat{t}-m_{t}^{2}} \\
E^{\prime}(\hat{s}, \hat{t}, \hat{u}) & =\left[\frac{1}{4}\left(\frac{\hat{u} \hat{t}}{M_{W^{\prime}}^{4}}-1\right)\left(1+4 \frac{M_{W^{\prime}}^{4}}{\hat{t}^{2}}\right)+\frac{\hat{s}}{M_{W^{\prime}}^{2}}\right]\left(\frac{\hat{t}}{\hat{t}-m_{t}^{2}}\right)^{2}
\end{aligned}
$$

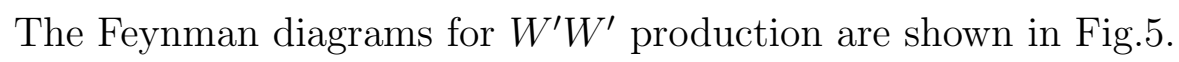

Other new vector pairs are $Z^{\prime} \gamma, Z^{\prime} Z^{\prime}$, and $W W^{\prime}$. All three of these processes 
involve only the $t$-channel. The matrix element for the first two processes can be generically written as

$$
\left.\sum|\mathcal{M}|^{2}\left(q \bar{q} \rightarrow Z^{\prime} \gamma\right)=2\left(e Q_{q}\right)^{2}\left[\left(g_{L}^{\prime q}\right)^{2}+\left(g_{R}^{\prime q}\right)^{2}\right)\right]\left[\frac{\hat{s}^{2}+M_{Z^{\prime}}^{4}}{2 \hat{t} \hat{u}}-1\right]
$$

and

$$
\sum|M|^{2}\left(\bar{q} q \rightarrow Z^{\prime} Z^{\prime}\right)=\left[\left(g_{L}^{\prime q}\right)^{4}+\left(g_{R}^{\prime q}\right)^{4}\right]\left[\frac{\hat{t}}{\hat{u}}+\frac{\hat{u}}{\hat{t}}+\frac{4 M_{Z^{\prime}}^{2} \hat{s}}{\hat{u} \hat{t}}-M_{Z^{\prime}}^{4}\left(\frac{1}{\hat{t}^{2}}+\frac{1}{\hat{u}^{2}}\right)\right]
$$

where $q=u_{L, R}, d_{L, R}, g_{L, R}^{\prime q}$ is the left- or right-handed coupling of the quark pair to the $Z^{\prime}$ boson, and $e Q_{q}$ is the coupling of the quark pair to $\gamma$. The analogous SM processes are given in the appendix.

The $W W^{\prime}$ pair production consists of the $t$-channel exchange of a top quark between the bottom and down quarks, and has a matrix element squared of

$$
\sum|M|^{2}\left(q_{i} q_{j} \rightarrow W W^{\prime}\right)=\left[\left(\frac{e}{\sqrt{2} s_{W}} V_{t q_{i}}\right)\left(\frac{g_{2}^{\prime}}{\sqrt{2}}\right)\right]^{2} 4 E^{\prime}(\hat{s}, \hat{t}, \hat{u})
$$

where $q_{i}=b, d, q_{j}=d_{R}, \bar{d}_{R}$ and $V_{t q_{i}}$ is the CKM mixing matrix element.

\subsection{Top Pair Asymmetry and Collider Signals}

The asymmetry $A_{F B}^{p \bar{p}}$ in the $p \bar{p}$ center-of-mass frame is defined as

$$
A_{F B}^{p \bar{p}}=\frac{N(\Delta y>0)-N(\Delta y<0)}{N(\Delta y>0)+N(\Delta y<0)}
$$

where $\Delta y=y_{t}-y_{\bar{t}}$ is the difference in rapidities of the top and anti-top quark. The axial couplings of the $W^{\prime}$ will contribute to a parity violation in $p \bar{p} \rightarrow t \bar{t}$. In order 
to assess the impact on top-pair measurements at the Tevatron, we implemented our model into MadGraph/MadEvent 4.4.24 [36], using CTEQ6.6M parton distribution functions [37] with factorization and renormalization scales $\mu_{F}=\mu_{R}=m_{t}$ [38]. We took $m_{t}=173.1 \mathrm{GeV}$ [39] [40] and applied a uniform SM K-factor of $K=1.31$ [20] to approximate the higher order QCD corrections for $\left(\mathrm{NNLO}_{\text {approx. }}\right) /(\mathrm{LO})$ as shown in [20]. We computed the total cross section $\sigma(t \bar{t})$ for top-pair production (Fig. 7(a)), $A_{F B}^{p \bar{p}}$ (Fig. 7(b) , and $M_{t \bar{t}}$ distribution (Fig. 8) for varying $M_{W^{\prime}}, M_{Z^{\prime}}=900 \mathrm{GeV}$, $\Gamma_{Z^{\prime}}=10.5 \mathrm{GeV}$, and $g_{2}^{\prime}=1$. Both $\sigma(t \bar{t})$ and $A_{F B}^{p \bar{p}}$ increase with larger $g_{2}^{\prime}$ (Table 3). 1

After accounting for the $\alpha_{s}^{3}$ SM contribution to the asymmetry, we are looking for a new physics asymmetry of $A_{F B}^{p \bar{p}}(N P)+0.080=0.19 \pm 0.07$ while reproducing the total cross section $\sigma(t \bar{t})=8.1 \pm 0.93 \mathrm{pb}$ [19]. A comparison of Fig. 7(a) and

Table 2: Reduced $\chi_{\text {red }}^{2}$ values for the CDF data versus predictions from the ALRM and the SM

\begin{tabular}{|c|c|c|}
\hline & $M_{Z^{\prime}}=900 \mathrm{GeV}$ & $\mathrm{SM}$ \\
\hline \hline$A_{F B}^{p p}$ & 0.09 & 4.08 \\
\hline$\sigma(t \bar{t})$ & 0.20 & 0.004 \\
\hline$d \sigma / d M_{t \bar{t}}$ & 0.84 & 0.44 \\
\hline
\end{tabular}

Fig. 7(b) shows that $M_{Z^{\prime}} \simeq 900 \mathrm{GeV}$ is compatible with the measured cross-section and $A_{F B}^{p \bar{p}}(N P)$ values; the ALRM results of $\sigma(t \bar{t})=7.7 \mathrm{pb}$ and $A_{F B}^{p \bar{p}}(N P)=0.09$ fall within $1 \sigma$ of experimental values. Fig. 8 shows the invariant mass distribution

\footnotetext{
${ }^{1}$ The choice of $M_{Z^{\prime}}=900 \mathrm{GeV}$ avoids the CDF limits on $Z^{\prime}$ production in the muon-pair and top-pair channels. [3] 41
} 
Table 3: Comparison of the effects of the value of $g_{2}^{\prime}$

\begin{tabular}{|c|c|c|c|c|c|}
\hline & $g_{2}^{\prime}=0$ & $g_{2}^{\prime}=0.5$ & $g_{2}^{\prime}=1$ & $g_{2}^{\prime}=1.5$ & $g_{2}^{\prime}=2$ \\
\hline \hline$A_{F B}^{p p}$ & 0.08 & 0.09 & 0.17 & 0.48 & 0.69 \\
\hline$\sigma(t \bar{t})[\mathrm{pb}]$ & 8.10 & 7.49 & 7.68 & 11.6 & 25.2 \\
\hline
\end{tabular}

for $M_{Z^{\prime}}=900 \mathrm{GeV}$. Table 2 lists the reduced chi-square values for the various measurements; since the errors are correlated, we do not make best fits to the combined data. Due to the weighting by the parton distribution functions, the $u \bar{u}$ fusion contributions to the cross-section dominant over the $d \bar{d}$ fusion contributions by a factor of about 5. However, the $W^{\prime}$ exchange in $d \bar{d}$ fusion is the dominant source of the forward-backward asymmetry (Table 4). The predicted $d \sigma / d \Delta y$ distribution of the ALRM is shown in Fig.6. The new physics contribution to the cross-section comes mostly from $d_{R} \bar{d}_{R}$.

Table 4: Cross-sections [pb] of $q \bar{q} \rightarrow t \bar{t}$ for $\Delta y>0$ and $\Delta y<0$, where $\Delta y=y_{t}-y_{\bar{t}}$ in the ALRM with $M_{Z^{\prime}}=900 \mathrm{GeV}$ and $g_{2}^{\prime}=1$. The subprocess CM energy is fixed at $\sqrt{\hat{s}}=500 \mathrm{GeV}, \mu_{F}=\mu_{R}=M_{t}=173.1 \mathrm{GeV}$. The QCD contributions to $A_{F B}$ are not included.

\begin{tabular}{|c|c|c|c|c|}
\hline$q \bar{q}$ & $\sigma[\mathrm{pb}]$ & $\sigma(\Delta y>0)$ & $\sigma(\Delta y<0)$ & $A_{F B}$ \\
\hline \hline$d_{L} \bar{d}_{L}$ & 39.7 & 20.1 & 19.3 & $\sim 0$ \\
\hline$d_{R} d_{R}$ & 70.7 & 67.4 & 3.07 & 0.92 \\
\hline$u_{L} \bar{u}_{L}$ & 40.0 & 20.0 & 19.6 & $\sim 0$ \\
\hline$u_{R} \bar{u}_{R}$ & 39.5 & 19.4 & 19.8 & $\sim 0$ \\
\hline$g g$ & 19.5 & 9.63 & 9.75 & 0 \\
\hline
\end{tabular}




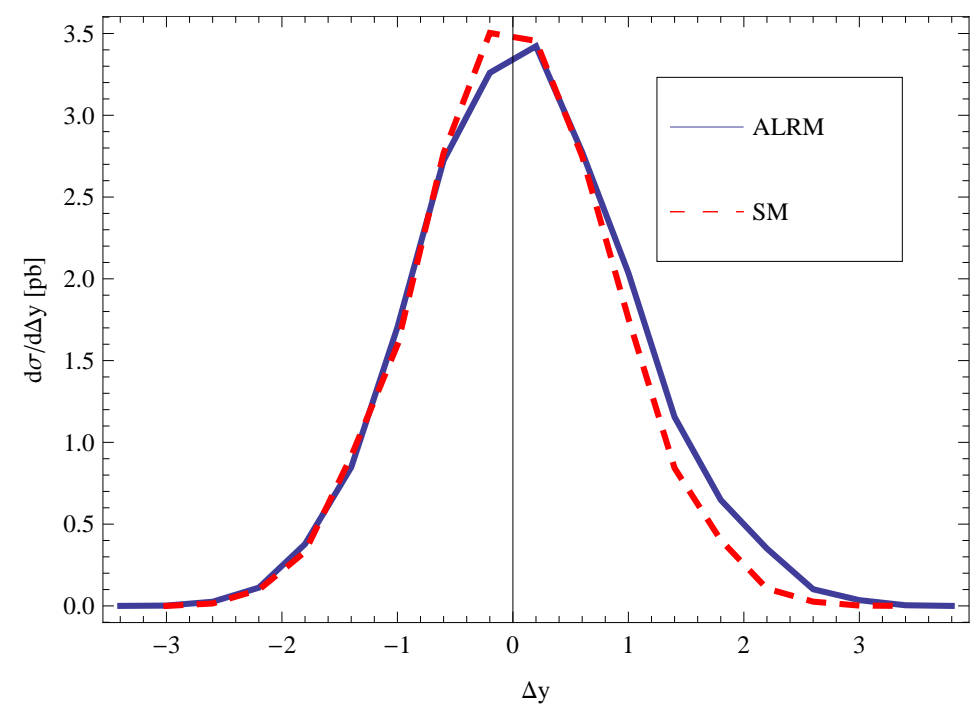

Figure 6: Comparison of the $\Delta y=y_{t}-y_{\bar{t}}$ distribution in the $p \bar{p}$ frame of the ALRM (solid) with $M_{Z^{\prime}}=900 \mathrm{GeV}, M_{W^{\prime}}=175 \mathrm{GeV}$, and $g_{2}^{\prime}=1$ with the SM (dashed) at the Tevatron.

The cross-sections for $W^{\prime}$ pair production and $Z^{\prime} \gamma$ production at the Tevatron are small compared to that of the corresponding SM $W$ processes. We find $\sigma\left(W^{\prime+} W^{\prime-}\right) / \sigma\left(W^{+} W^{-}\right)=0.03$ and $\sigma\left(Z^{\prime}+\gamma\right) / \sigma(Z+\gamma)=1.29 \times 10^{-5}$ for $M_{Z^{\prime}}=900$ $\mathrm{GeV}$ and $g_{2}^{\prime}=1$

Fig. 9 shows the cross-sections for various pairs of vector bosons versus CM energy at the LHC. The SM $W W, Z Z$, and $Z \gamma$ cross-sections are shown for reference. The expected cross-sections at the Tevatron and LHC for these processes are given in Table 5 . 


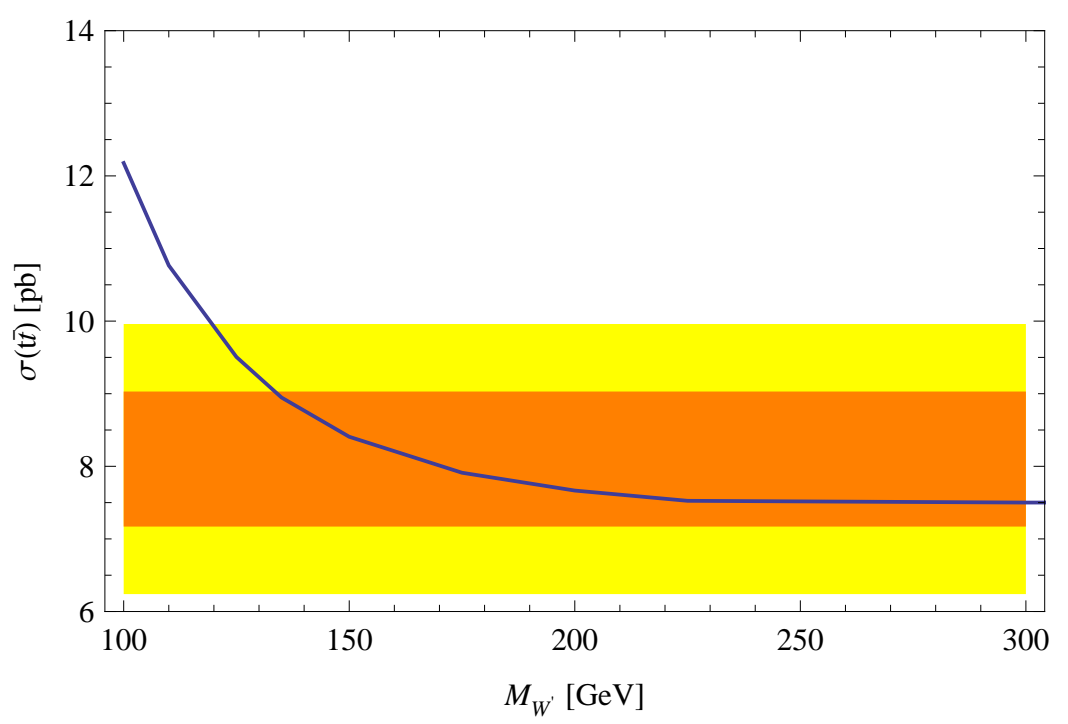

(a) $\sigma(p \bar{p} \rightarrow t \bar{t})$

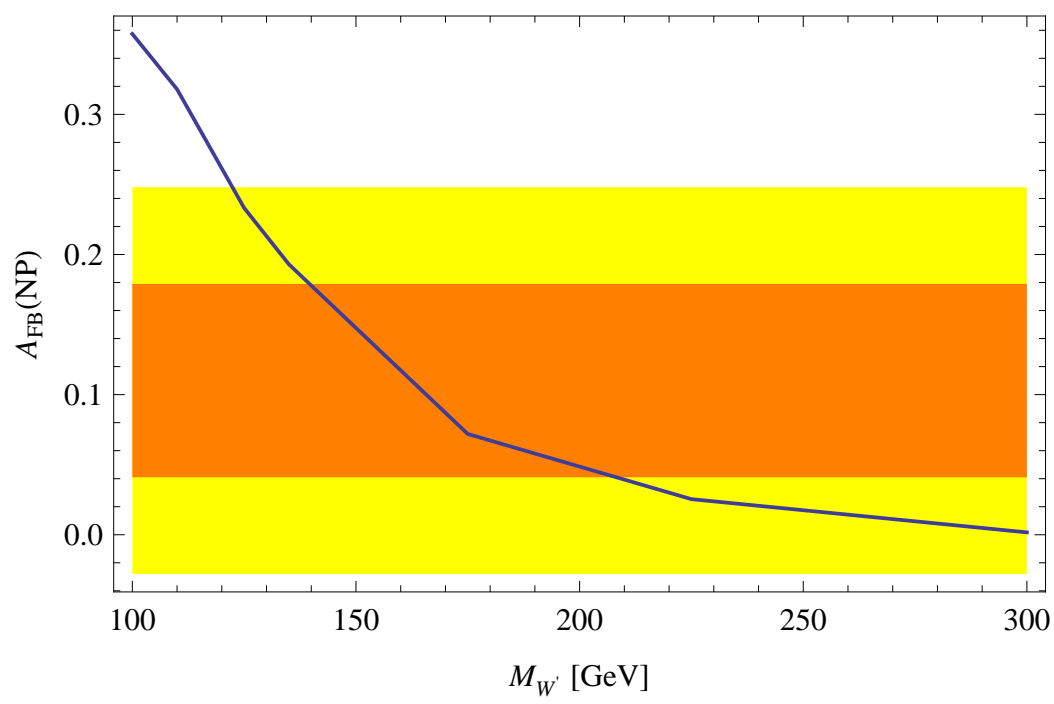

(b) $A_{F B}^{p \bar{p}}(N P)$

Figure 7: (a) $\sigma(t \bar{t})[\mathrm{pb}]$ and (b) $A_{F B}^{p \bar{p}}(N P)$ versus $M_{W^{\prime}}$ in the ALRM with $1 \sigma$ (orange) and $2 \sigma$ (yellow) CDF bounds. 


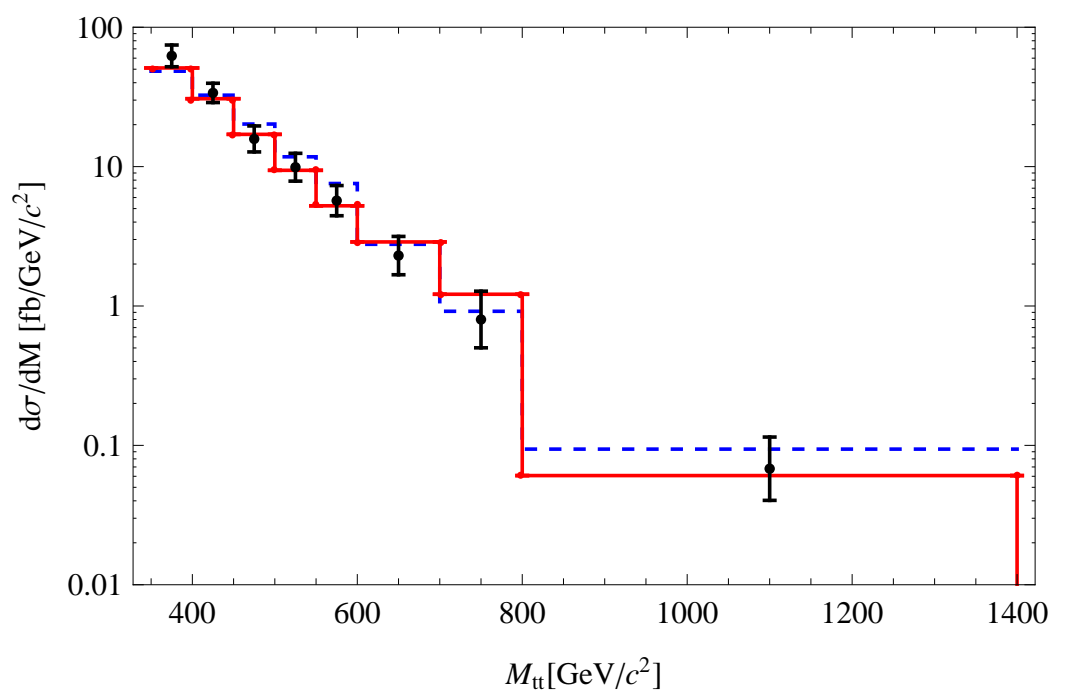

Figure 8: $M_{t \bar{t}}$ distribution of CDF data [3], MadEvent SM (red solid), and ALRM (blue dashed ). $K=1.31$ has been included uniformly across all bins.

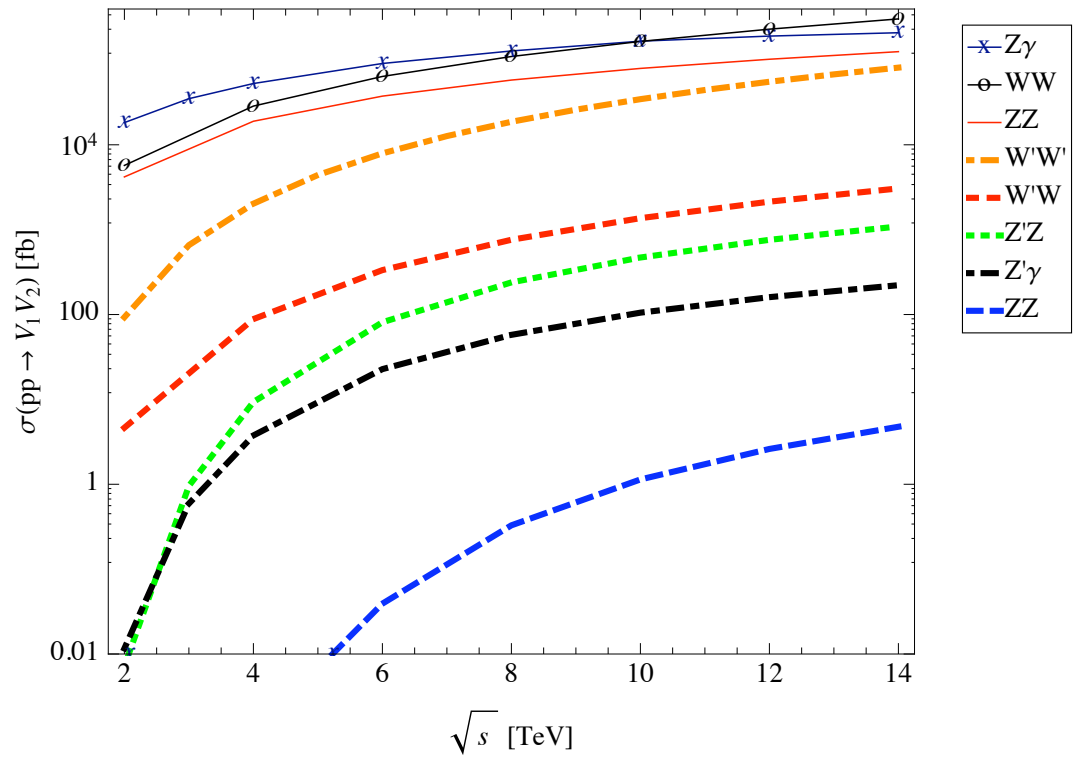

Figure 9: Cross-sections [fb] vs. $\sqrt{s}$ for various vector boson pairs in $p p$ collisions. 
Table 5: Expected values for $\sigma\left(V_{1} V_{2}\right)[\mathrm{pb}]$ at the Tevatron $(\sqrt{s}=2 \mathrm{TeV})$ and the LHC $(\sqrt{s}=14 \mathrm{TeV}) . K=1.31$ has been included

\begin{tabular}{|c|c|c|c|c|c|c|c|c|}
\hline & $W^{+} W^{-}$ & $W^{\prime+} W^{\prime-}$ & $Z^{\prime} \gamma$ & $Z^{\prime} Z^{\prime}$ & $W W^{\prime}$ & $Z \gamma$ & $Z Z$ & $Z Z^{\prime}$ \\
\hline \hline Tevatron & 15.12 & 0.49 & 0.003 & $\sim 0$ & 0.02 & 23.33 & 8.52 & $\sim 0$ \\
\hline LHC & 304.30 & 81.14 & 0.17 & 0.005 & 3.06 & 159.67 & 125.16 & 1.09 \\
\hline
\end{tabular}

\section{$2.4 W^{\prime}$ and $Z^{\prime}$ Decays}

In the limit that the mixings of the new gauge bosons with the SM weak bosons are small, their decays are governed by the interactions in Eq. 2.7 and Table 1. We assume that $m_{t}<M_{W^{\prime}}<200 \mathrm{GeV}$. Therefore, the $W^{\prime}$ decays to a top quark that is almost at rest with respect to the $W^{\prime}$. For $M_{W^{\prime}}=175 \mathrm{GeV}$ and $g_{2}^{\prime}=1$, the width for $W^{\prime}$ decay is

$$
\Gamma\left(W^{\prime} \rightarrow t \bar{d}\right)=\frac{g_{2}^{\prime 2}}{(16 \pi)} M_{W^{\prime}}\left(1-\frac{3 m_{t}^{2}}{2 M_{W^{\prime}}^{2}}+\frac{m_{t}^{6}}{2 M_{W^{\prime}}^{6}}\right)=20.7 \mathrm{MeV}
$$

This small partial width is due to the limited phase space. For $M_{Z^{\prime}}=900 \mathrm{GeV}$ and $g_{2}^{\prime}=1$, the partial widths for the leading $Z^{\prime}$ decays are

$$
\begin{gathered}
\Gamma\left(Z^{\prime} \rightarrow u \bar{u}\right)=\left(\frac{5 g_{1}^{\prime 2}}{6 g^{\prime}}\right)^{2} \frac{M_{Z^{\prime}}}{4 \pi}=0.64 \mathrm{GeV} \\
\Gamma\left(Z^{\prime} \rightarrow d \bar{d}\right)=\left[\left(\frac{g_{1}^{\prime 2}}{6 g^{\prime}}\right)^{2}+\left(\frac{g_{1}^{\prime 2}}{3 g^{\prime}}-\frac{g^{\prime}}{2}\right)^{2}\right] \frac{M_{Z^{\prime}}}{8 \pi}=8.60 \mathrm{GeV} \\
\Gamma\left(Z^{\prime} \rightarrow l \bar{l}\right)=\left(\frac{3 g_{1}^{\prime 2}}{2 g^{\prime}}\right)^{2} \frac{M_{Z^{\prime}}}{8 \pi}=0.86 \mathrm{GeV}
\end{gathered}
$$


The $Z^{\prime}$ can be singly produced in the s-channel at the Tevatron, but the the dijet signal from its decays will be difficult to identify above the large QCD dijets background. One can set a lower limit on the $Z^{\prime}$ mass from dimuon and $t \bar{t}$ production. The $M_{t \bar{t}}$ distribution gives a more stringent limit, $M_{Z^{\prime}}^{A L R M} \gtrsim 800 \mathrm{GeV}$, than the dimuon data give.

\subsection{Summary}

We have discussed the Asymmetric Left-Right Model (ALRM) based on the $U^{\prime}(1) \times$ $S U^{\prime}(2) \times S U(2)$ gauge group. The symmetry is broken spontaneously, first by a Higgs doublet of the prime sector to $U_{Y}$, and then by another Higgs doublet in the SM sector. The $S U^{\prime}(2)$ acts on a $(t, d)_{R}$ doublet. The ALRM includes a $W^{\prime}$ boson with the $(t, d)_{R}$ coupling, and a $Z^{\prime}$ boson with diagonal $u \bar{u}$, $d \bar{d}$, and $t \bar{t}$ couplings. With $M_{W^{\prime}} \approx 175 \mathrm{GeV}$ and $M_{Z^{\prime}} \approx 900 \mathrm{GeV}$, the ALRM can explain the $A_{F B}^{p \bar{p}}$ measurement at the Tevatron, while remaining consistent with the $\sigma(t \bar{t})$ and $t \bar{t}$ mass distribution [15] [19] [3]. We have evaluated the cross-sections for the production of vector boson pairs at the LHC. Since the $W^{\prime}$ decays only to quarks, its collider signal may be difficult to distinguish from SM backgrounds. However, small mixings with the $W$ will lead to small leptonic branching fractions that should allow it to be more readily probed at the LHC. The $Z^{\prime}$ can be probed via dilepton and $t \bar{t}$ production. 


\section{Chapter 3}

\section{Tevatron Asymmetry of Tops in a}

\section{W', Z' Model}

\subsection{Introduction}

The top-pair forward-backward asymmetry at the Tevatron collider by the CDF collaboration [13] has generated much interest [29, 30, 31, 32, 33, 34, 42, 43, 44, 45, 46, 47, 48, as a possible harbinger of new physics. At lowest order in the SM, the toppair production is symmetric under charge conjugation. A small forward-backward asymmetry of $A_{F B}=0.06 \pm 0.01$ [49, 24, 17] in the $t \bar{t}$ rest-frame arises through the interference of NLO QCD processes that differ under charge conjugation. Recent results from the CDF Collaboration [2] using a data set of $5.3 \mathrm{fb}^{-1}$ show that the forward-backward asymmetry in top pair production $A_{F B}$ still deviates from SM predictions and, furthermore, is mass-dependent. In particular, the asymmetry is most prominent in regions of high rapidity difference $|\Delta y|>1$ and large invariant mass $M_{t \bar{t}}>450 \mathrm{GeV}$, where there are $2 \sigma$ and $3 \sigma$ deviations, respectively, from NLO predictions. Such a distribution is a generic feature in the $t$-channel exchange of 
a particle [50] [51] [26] [27]. In the previous chapter, we discussed the Asymmetric Left-Right Model (ALRM) based on the gauge symmetry $U^{\prime}(1) \times S U^{\prime}(2) \times S U(2)$. This model has electroweak $W^{\prime}$ and $Z^{\prime}$ bosons in addition to the $W$ and $Z$ of the SM. In that work, we did not assume a specific Higgs mechanism, but treated the $W^{\prime}$ and $Z^{\prime}$ masses to be independent parameters. In this chapter, we assume that the primed gauge group is broken by the neutral member of a triplet Higgs sector by a vacuum expectation value (vev) $v^{\prime}$. The SM electroweak gauge group is broken as usual by the vev $v$ of the neutral member of the SM Higgs doublet. In the ALRM, the $W^{\prime}$ boson has a $(t, d)$ right-handed coupling with coupling $g_{2}^{\prime}$ and the $Z^{\prime}$ boson has diagonal fermion couplings. We determine the model parameters from the CDF data to be $M_{W^{\prime}}=700 \mathrm{GeV}, M_{Z^{\prime}}=1 \mathrm{TeV}$, and $g_{2}^{\prime}=3$. The $W^{\prime}$ and $Z^{\prime}$ total decay widths are then of $\mathcal{O}(100 \mathrm{GeV})$.

We begin with an overview of the ALRM and then discuss the symmetry breaking in Section II. In Section III, we calculate the forward-backward asymmetry $A_{F B}$ as functions of $|\Delta y|$ and $M_{t \bar{t}}$ and compare our results with the CDF data. In Section IV, we evaluate signals of the ALRM at the LHC. The signals at the LHC from $Z^{\prime}, W^{\prime}$ production and their $Z^{\prime} \rightarrow t \bar{t}, W^{\prime} \rightarrow t b$ decays can definitively test this model interpretation of the CDF top asymmetry data. We end with our conclusions in Section V. 


\subsection{Asymmetric Left-Right Model}

The ALRM begins with the gauge group $U^{\prime}(1) \times S U^{\prime}(2) \times S U(2)$. The unprimed $S U(2)$ acts on the usual SM left-handed quark doublets. The primed $S U^{\prime}(2)$ applies to the right-handed doublet $(t, d)_{R}^{T}$ in an unconventional grouping.

The gauge symmetries are broken sequentially, starting with $U^{\prime}(1) \times S U^{\prime}(2) \rightarrow U_{Y}$ to obtain the SM hypercharge $\frac{Y}{2}=T_{3}^{\prime}+\frac{Y^{\prime}}{2}$, and then $U_{Y}(1) \times S U(2) \rightarrow U_{E M}$ to obtain $Q=T_{3}+\frac{Y}{2}$. By using this sequential approximation to the breaking, we preserve the SM interaction.

After symmetry breaking, there are massive $Z^{\prime}$ and $W^{\prime \pm}$ bosons in addition to the usual weak bosons. The $W^{\prime \pm}$ have a $Z^{\prime} W^{\prime} W^{\prime}$ tri-gauge boson coupling given by $g_{2}^{\prime 2} / \sqrt{g^{\prime 2}+g_{1}^{\prime 2}}$, which is of order $\mathcal{O}(1)$. In order to preserve unitarity, the SM $Z$ also appears in the vertex $Z W^{\prime+} W^{\prime-}$ with coupling $-e \tan \theta_{W}$. Additional massive fermions will be needed for anomaly cancellation.

Our results are derived by making two successive rotations of gauge boson states. First, we make the rotation

$$
B=\left(g_{2}^{\prime} B^{\prime}+g_{1}^{\prime} W^{\prime 3}\right) / \sqrt{g_{1}^{\prime 2}+g_{2}^{\prime 2}}, Z^{\prime}=\left(-g_{1}^{\prime} B^{\prime}+g_{2}^{\prime} W^{\prime 3}\right) / \sqrt{g_{1}^{\prime 2}+g_{2}^{\prime 2}} .
$$

Then the couplings to the generators become

$$
g_{1}^{\prime} \frac{Y^{\prime}}{2} B^{\prime}+g_{2}^{\prime} T_{3}^{\prime} W_{3}^{\prime}=\left(\frac{g_{1}^{\prime} g_{2}^{\prime}}{\sqrt{g_{1}^{\prime 2}+g_{2}^{\prime 2}}}\right) \frac{Y}{2} B+\sqrt{g_{1}^{\prime 2}+g_{2}^{\prime 2}}\left(T_{3}^{\prime}-\frac{g_{1}^{\prime 2} \frac{Y}{2}}{g_{1}^{\prime 2}+g_{2}^{\prime 2}}\right) Z^{\prime}
$$

Subsequently, we perform the usual SM rotation from the basis of $B, W^{3}$ to the basis of $A, Z$. To simplify the expressions, we denote $g^{\prime}=\sqrt{g_{2}^{\prime 2}+g_{1}^{\prime 2}}$. The SM hypercharge 
coupling is $g_{1}=g_{1}^{\prime} g_{2}^{\prime} / g^{\prime}=e / c_{W}$, and the SM $S U(2)$ coupling is $g_{2}=e / s_{W}$. It is also useful to note that $g_{1}^{\prime}=\left(c_{W}^{2} / e^{2}-g_{2}^{\prime-2}\right)^{-\frac{1}{2}}$.

\subsection{1 $W^{\prime}, Z^{\prime}$ Mass Relation}

We assume that the Higgs mechanism is due to the condensate of a primed Higgs triplet with $T^{\prime}=1$, which is denoted as $\phi^{\prime}$ with its vev as $\left(0,0, v^{\prime} / \sqrt{2}\right)^{T}$. Using a Higgs triplet allows for a larger mass gap between the $Z^{\prime}$ and $W^{\prime}$ bosons than a Higgs doublet. Therefore,

$$
\frac{1}{2} M_{Z^{\prime}}^{2}=\left\langle\phi^{\prime \dagger}\left(g^{\prime} T_{3}^{\prime}\right)^{2} \phi^{\prime}\right\rangle, \quad M_{Z^{\prime}}=g^{\prime} v^{\prime}
$$

and

$$
M_{W^{\prime}}^{2}=\left\langle\phi^{\prime \dagger} T_{-}^{\prime}\left(g_{2} / \sqrt{2}\right)^{2} T_{+}^{\prime} \phi^{\prime}\right\rangle, \quad M_{W^{\prime}}=\frac{1}{\sqrt{2}} g_{2}^{\prime} v^{\prime}
$$

The two-stage approximation is justified by $M_{Z^{\prime}}, M_{W^{\prime}} \gg M_{Z}, M_{W}$. Thus,

$$
\frac{M_{W^{\prime}}}{M_{Z^{\prime}}}=\frac{g_{2}^{\prime}}{\sqrt{2} g^{\prime}}=\sqrt{\frac{g_{2}^{\prime 2}-e^{2} / c_{W}^{2}}{2 g_{2}^{\prime 2}}}
$$

A search by CDF in the muon-pair channel established a lower bound of $M_{Z^{\prime}}>$ $1051 \mathrm{GeV}$ for a $Z^{\prime}$ with SM couplings [52]. Dijet searches have placed exclusion limits of $320<M_{Z^{\prime}}<740 \mathrm{GeV}$, again with SM coupling of the $Z^{\prime}$ [53]. For our $Z^{\prime}$, with $g_{2}^{\prime}=3$, the dimuon limit is $M_{Z^{\prime}} \gtrsim 500 \mathrm{GeV}$ while the dijets do not limit $M_{Z^{\prime}}$. We plot the relationship between $M_{W^{\prime}}, M_{Z^{\prime}}$, and $g_{2}^{\prime}$ in Fig, 10. Numerically, we use $e^{2}=4 \pi \alpha\left(M_{Z}\right)=0.30$ and $c_{W}^{2}=1-0.23=0.77$. After accounting for constraints from experimental data, we use $M_{W^{\prime}}=700 \mathrm{GeV}, M_{Z^{\prime}}=1 \mathrm{TeV}$ and $g_{2}^{\prime}=3$ as our 
benchmark point. Note that for $g_{2}^{\prime}>1$, the $M_{W^{\prime}} / M_{Z^{\prime}}$ ratio is relatively insensitive to the value of $g_{2}^{\prime}$ and it is approximately given by the large $g_{2}^{\prime}$ limit of $M_{W^{\prime}}=M_{Z^{\prime}} / \sqrt{2}$.

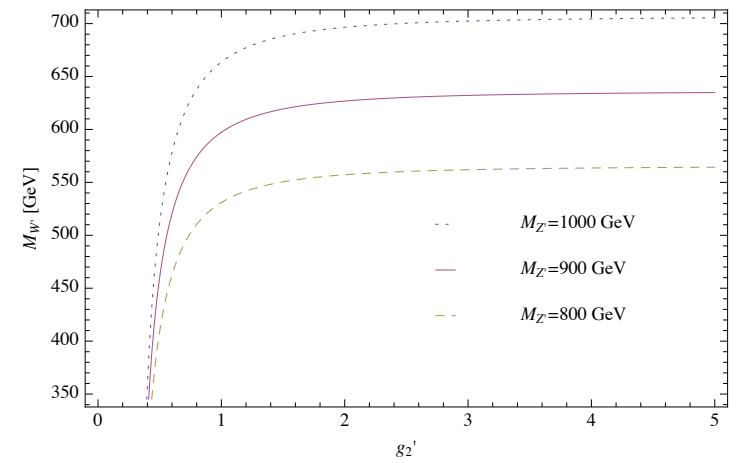

(a) $M_{W^{\prime}}$ vs. $g_{2}^{\prime}$ for $M_{Z^{\prime}}=800,900,1000 \mathrm{GeV}$.

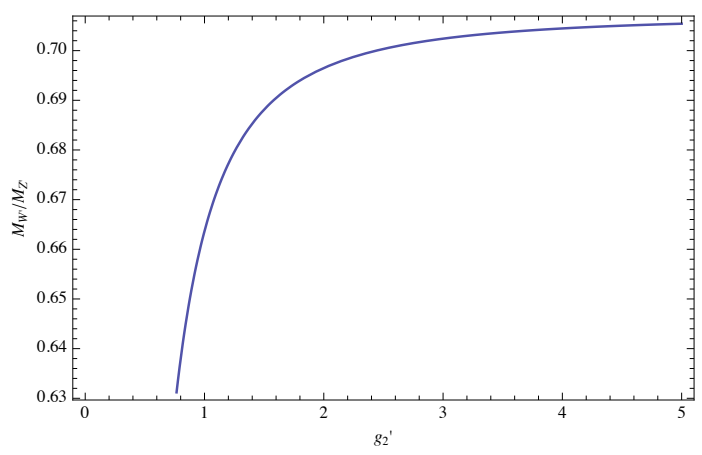

(b) $M_{W^{\prime}} / M_{Z^{\prime}}$ vs. $g_{2}^{\prime}$

Figure 10: Dependence of $M_{W^{\prime}}$ on $g_{2}^{\prime}$ and $M_{Z^{\prime}}$. The relationship is given by Eq. 3.5

\subsection{2 $W^{\prime}$ and $Z^{\prime}$ decays}

For $M_{W^{\prime}}=700 \mathrm{GeV}$ and $g_{2}^{\prime}=3$, the width for $W^{\prime}$ decay is

$$
\Gamma\left(W^{\prime} \rightarrow t \bar{d}\right)=\frac{g_{2}^{\prime 2}}{(16 \pi)} M_{W^{\prime}}\left(1-\frac{3 m_{t}^{2}}{2 M_{W^{\prime}}^{2}}+\frac{m_{t}^{6}}{2 M_{W^{\prime}}^{6}}\right)=114 \mathrm{GeV}
$$


For $M_{Z^{\prime}}=1 \mathrm{TeV}$ and $g_{1}^{\prime}=0.35$, the partial widths for the $Z^{\prime}$ decays are

$$
\begin{aligned}
\Gamma\left(Z^{\prime} \rightarrow d \bar{d}\right) & =\left[\left(\frac{g_{1}^{\prime 2}}{6 g^{\prime}}\right)^{2}+\left(\frac{g_{1}^{\prime 2}}{3 g^{\prime}}-\frac{g^{\prime}}{2}\right)^{2}\right] \frac{M_{Z^{\prime}}}{8 \pi}=89 \mathrm{GeV} \\
\Gamma\left(Z^{\prime} \rightarrow t \bar{t}\right) & =\frac{M_{Z^{\prime}}}{64 \pi}\left[\left(g^{\prime}-\frac{5 g_{1}^{\prime 2}}{3 g^{\prime}}\right)^{2}\left(1+\frac{2 m_{t}^{2}}{M_{Z^{\prime}}^{2}}\right)\right. \\
& \left.+\left(g^{\prime}-\frac{g_{1}^{\prime 2}}{g^{\prime}}\right)^{2}\left(1-\frac{4 m_{t}^{2}}{M_{Z^{\prime}}^{2}}\right)\right] \times \sqrt{1-\frac{4 m_{t}^{2}}{M_{Z^{\prime}}^{2}}}=79 \mathrm{GeV} \\
\Gamma\left(Z^{\prime} \rightarrow l \bar{l}\right) & =\left(\frac{3 g_{1}^{\prime 2}}{2 g^{\prime}}\right)^{2} \frac{M_{Z^{\prime}}}{8 \pi}=0.1 \mathrm{GeV} \\
\Gamma\left(Z^{\prime} \rightarrow u \bar{u}\right) & =\left(\frac{5 g_{1}^{\prime 2}}{6 g^{\prime}}\right)^{2} \frac{M_{Z^{\prime}}}{8 \pi}=0.04 \mathrm{GeV} \\
\Gamma\left(Z^{\prime} \rightarrow \nu \bar{\nu}\right) & =\left(\frac{g_{1}^{\prime 2}}{2 g^{\prime}}\right)^{2} \frac{M_{Z^{\prime}}}{8 \pi}=0.01 \mathrm{GeV}
\end{aligned}
$$

The total $Z^{\prime}$ width is $\Gamma_{Z^{\prime}}=168 \mathrm{GeV}$. The variation of the $Z^{\prime}$ width with $\hat{s}$ will be approximated in our calculations of $Z^{\prime}$ production by the prescription $M_{Z^{\prime}}^{2} \rightarrow \hat{s}$, where $\hat{s}$ is the subprocess CM energy. The branching ratios to a top-pair and leptons are

$$
\begin{aligned}
& \mathcal{B R}\left(Z^{\prime} \rightarrow t \bar{t}\right)=0.5 \\
& \mathcal{B R}\left(Z^{\prime} \rightarrow l \bar{l}\right)=6.0 \times 10^{-4}
\end{aligned}
$$

where $l$ is the sum of $e, \mu$, and $\tau$ modes in the width. The small leptonic width of the $Z^{\prime}$ along with a broad total width makes its detection in the Drell-Yan lepton channel difficult. 


\subsection{Results}

The asymmetry $A_{F B}$ in the $p \bar{p}$ center-of-mass frame is defined as

$$
A_{F B}=\frac{N(\Delta y>0)-N(\Delta y<0)}{N(\Delta y>0)+N(\Delta y<0)}
$$

where $\Delta y=y_{t}-y_{\bar{t}}$ is the difference in rapidities of the top and anti-top quark. We implemented the ALRM in MadGraph/MadEvent 4.4.44 [36], using CTEQ6.6M parton distribution functions [37] with factorization and renormalization scales $\mu_{F}=$ $\mu_{R}=m_{t}$ [38]. We took $m_{t}=173.1 \mathrm{GeV}$ [39] [40] and applied a uniform SM K-factor of $K=1.3$ to approximate the higher order QCD corrections for $\left(\mathrm{NNLO}_{\text {approx. }}\right) /(\mathrm{LO})$ as shown in [20].

We calculate the total cross-section $\sigma(t \bar{t})$, total $A_{F B}$, as well as $A_{F B}$ in $|\Delta y|<$ 1, $|\Delta y|>1, M_{t \bar{t}}<450 \mathrm{GeV}$ and $450<M_{t \bar{t}}<800 \mathrm{GeV}$ for various values of $g_{2}^{\prime}$ and $M_{W^{\prime}}$. We compare our results to the latest experimental results from the CDF collaboration [2] in Table 6 and in Figs 11] 12(a) 12(b), We find that the values of $g_{2}^{\prime}=3, M_{W^{\prime}}=700$, and $M_{Z^{\prime}}=1 \mathrm{TeV}$ provides an overall description of the data (see Table 7). Alternate values of $M_{W^{\prime}}$ and $g_{2}^{\prime}$ allow for a closer match in the high invariant mass bin at the expense of consistency with the measured crosssection. We do not attempt a global fit to the data since there are correlations amongst the experimental distributions. The exact diagonalization of the weak boson mass matrices and radiative corrections to $M_{W^{\prime}}$ may help alleviate this tension. We examine the dependence of $A_{F B}$ on $M_{t \bar{t}}$ in Fig,13. The shape of the curve is

dominated by the $Z^{\prime}$ contribution to the $d_{R} \bar{d}_{R} \rightarrow t \bar{t}$ subprocess, as shown in Fig. 14 . 
The structure function product $[x d(x)]^{2}$ has a maximum at $M_{t \bar{t}}=300 \mathrm{GeV}$.

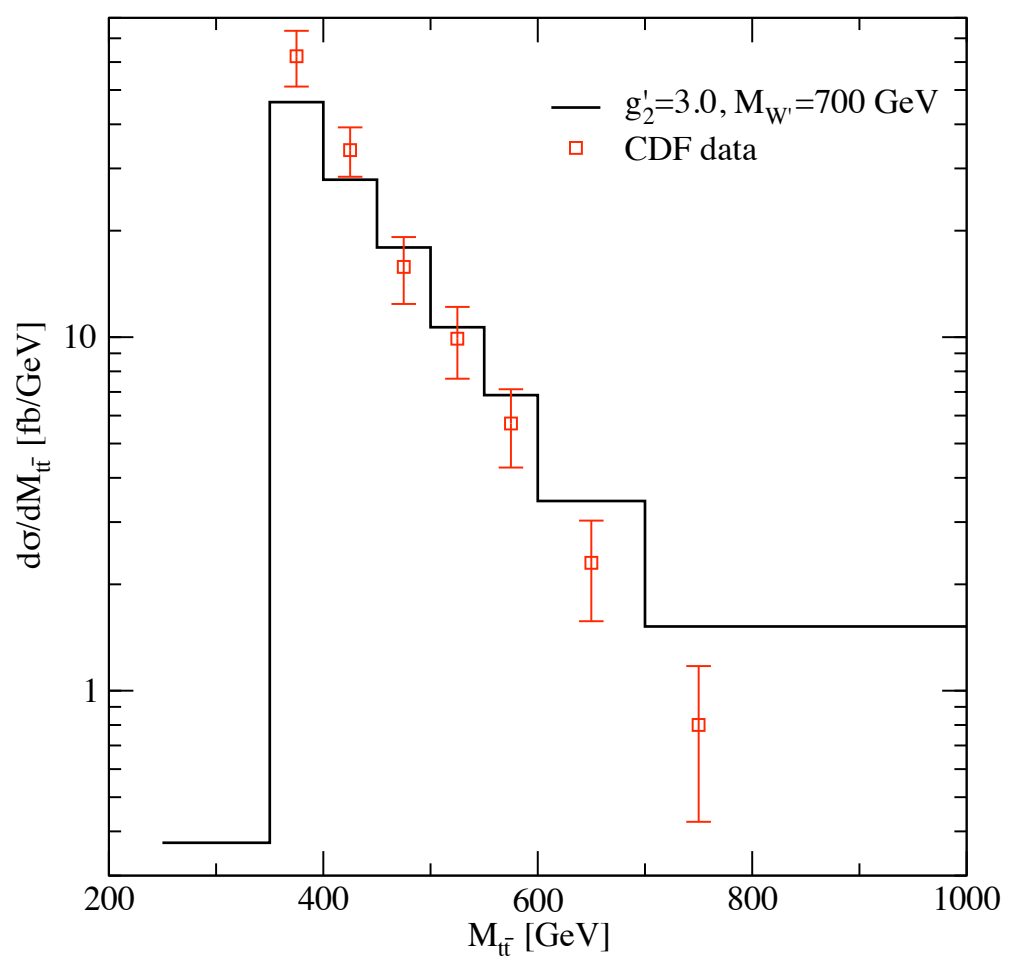

Figure 11: $d \sigma / d M_{t \bar{t}}$ distribution of CDF data(points with uncertainties) vs. ALRM (solid histograms) for the model benchmark point of $g_{2}^{\prime}=3.0, M_{W^{\prime}}=700 \mathrm{GeV}$, and $M_{Z^{\prime}}=1 \mathrm{TeV}$.

\subsection{Predictions at the LHC}

\subsubsection{Z' signatures}

The $Z^{\prime}$ in the ALRM with $\sim 1 \mathrm{TeV}$ mass and $\sim 200 \mathrm{GeV}$ width provides a promising route for early LHC discovery or exclusion. The $t \bar{t}$ invariant mass distribution should 
Table 6: ALRM predictions for the $t \bar{t}$ total cross-section $\sigma(t \bar{t})$, the forward-backward asymmetry in the $p \bar{p} \mathrm{CM}$ frame $\left(A_{F B}\right)$, and the cross-sections for the specified ranges of rapidity differences $\Delta y$ and $M_{t \bar{t}}$ invariant mass ranges. $M_{Z^{\prime}}$ is determined by Eq.3.5. A QCD correction factor $K=1.3$ is included in the cross-section calculation. The ALRM asymmetry numbers are the new physics contributions only and do not include the SM QCD contribution, so they should be compared with the final row in the table. The SM values are based on the MCFM study of Ref. [5]. The last row is the New Physics (NP) contribution inferred from the differences of data and SM entries.

\begin{tabular}{|c|c|c|c|c|c|}
\hline$g_{2}^{\prime}$ & $M_{W^{\prime}}[\mathrm{GeV}]$ & $\sigma(t \bar{t})[\mathrm{pb}]$ & $A_{F B}$ & $\begin{array}{c}A_{F B} \\
M_{t \bar{t}}<450 \mathrm{GeV}\end{array}$ & $\begin{array}{c}A_{F B} \\
450<M_{t \bar{t}}<800 \mathrm{GeV}\end{array}$ \\
\hline \hline 3.0 & 700 & 8.45 & 0.06 & -0.01 & 0.136 \\
3.5 & 700 & 9.05 & 0.11 & 0.01 & 0.22 \\
3.5 & 650 & 9.8 & 0.16 & 0.03 & 0.26 \\
3.0 & 550 & 10.4 & 0.22 & 0.04 & 0.33 \\
2.5 & 500 & 10.5 & 0.19 & 0.003 & 0.32 \\
\hline \hline Data [2][54] & & $7.70 \pm 0.52$ & $0.158 \pm 0.074$ & $-0.116 \pm 0.153$ & $0.475 \pm 0.122$ \\
SM & & $7.45_{-0.63}^{+0.72}$ & $0.058 \pm 0.009$ & $0.04 \pm 0.006$ & $0.088 \pm 0.0013$ \\
NP & & - & $0.100 \pm 0.074$ & $-0.156 \pm 0.147$ & $0.387 \pm 0.121$ \\
\hline \hline & & & & $A_{F B}$ & $A_{F B}$ \\
$g_{2}^{\prime}$ & $M_{W^{\prime}}[\mathrm{GeV}]$ & $\sigma(t \bar{t})[\mathrm{pb}]$ & $A_{F B}$ & $|\Delta y|<1$ & $|\Delta y|>1$ \\
\hline \hline 3.0 & 700 & 8.45 & 0.06 & 0.03 & 0.14 \\
3.5 & 700 & 9.05 & 0.11 & 0.06 & 0.26 \\
3.5 & 650 & 9.8 & 0.16 & 0.06 & 0.36 \\
3.0 & 550 & 10.4 & 0.22 & 0.09 & 0.42 \\
2.5 & 500 & 10.5 & 0.19 & 0.07 & 0.40 \\
\hline \hline Data [2] [54] & & $7.70 \pm 0.52$ & $0.158 \pm 0.074$ & $0.026 \pm 0.118$ & $0.611 \pm 0.256$ \\
SM & & $7.45_{-0.63}^{+0.72}$ & $0.058 \pm 0.009$ & $0.039 \pm 0.006$ & $0.123 \pm 0.018$ \\
NP & & - & $0.100 \pm 0.074$ & $0.387 \pm 0.112$ & $0.488 \pm 0.257$ \\
\hline
\end{tabular}




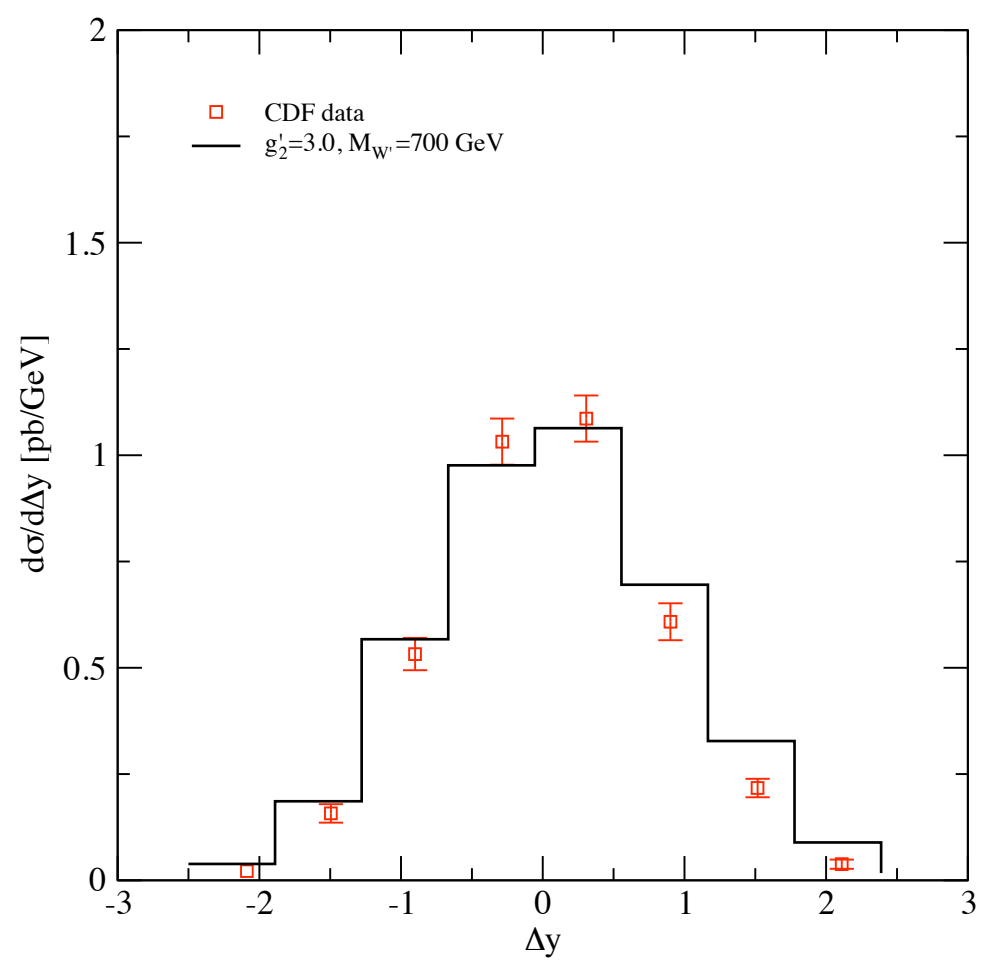

(a)

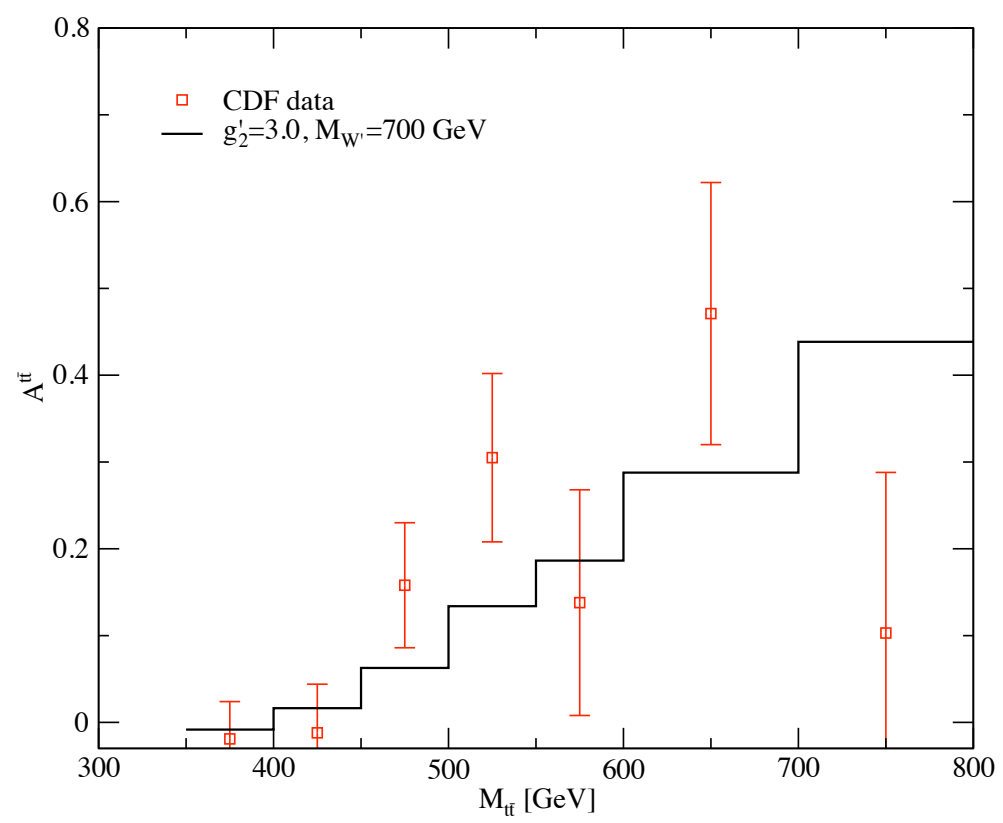

(b)

Figure 12: Comparison of CDF data vs. ALRM predictions in (a) $\Delta y$ distribution and (b) $A_{F B}$ in the $p \bar{p}$ CM frame vs. $M_{t \bar{t}}$ for the model benchmark point of $g_{2}^{\prime}=$ $3, M_{W^{\prime}}=700 \mathrm{GeV}$, and $M_{Z^{\prime}}=1 \mathrm{TeV}$. The points with the uncertainties are the CDF measurements and the solid histograms are the ALRM predictions including the SM QCD contribution. 
Table 7: $\chi^{2} /$ d.o.f. values for various $g_{2}^{\prime}$ and $M_{W^{\prime}}$ mass values using $A_{F B}$ in the $7 M_{t \bar{t}}$ bins and the total cross-section $\sigma(t \bar{t})$. We have included a $K$-factor of 1.3 for $\sigma(t \bar{t})$.

\begin{tabular}{|c|c||c|c|}
\hline$g_{2}^{\prime}$ & $M_{W^{\prime}}[\mathrm{GeV}]$ & $\chi^{2}\left(A_{F B}^{t t}\right) /$ bin & $\chi^{2}(\sigma(t \bar{t}))$ \\
\hline \hline 3.0 & 700 & 1.8 & 2.0 \\
3.5 & 700 & 1.2 & 6.8 \\
3.5 & 650 & 1.4 & 15.2 \\
3.0 & 550 & 1.3 & 27.8 \\
\hline
\end{tabular}

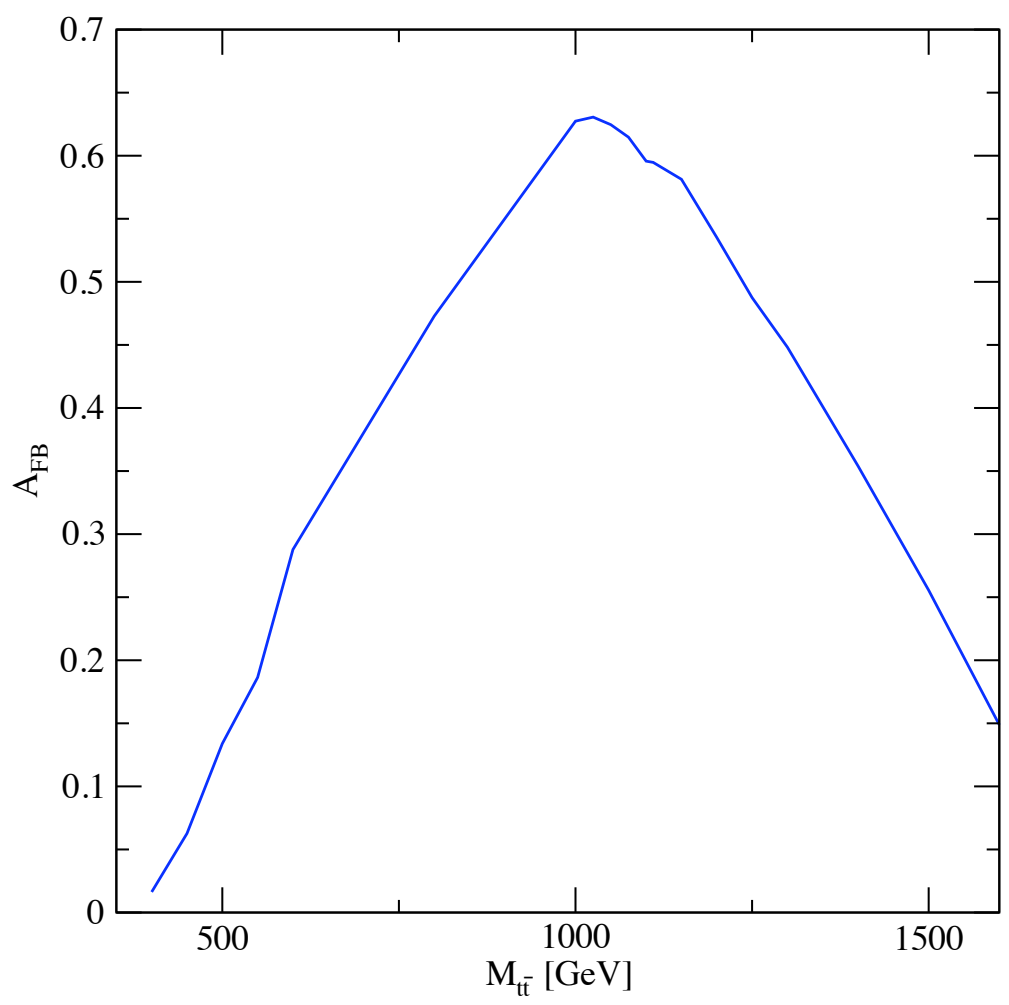

Figure 13: The asymmetry in the $t \bar{t} \mathrm{CM}$ frame vs. the invariant $t \bar{t}$ mass in the ALRM for $g_{2}^{\prime}=3.0, M_{W^{\prime}}=700 \mathrm{GeV}$, and $M_{Z^{\prime}}=1 \mathrm{TeV}$. The decrease in $A_{F B}$ above $1 \mathrm{TeV}$ is due to the dominance of the $Z^{\prime}$ contribution to the cross-section. 


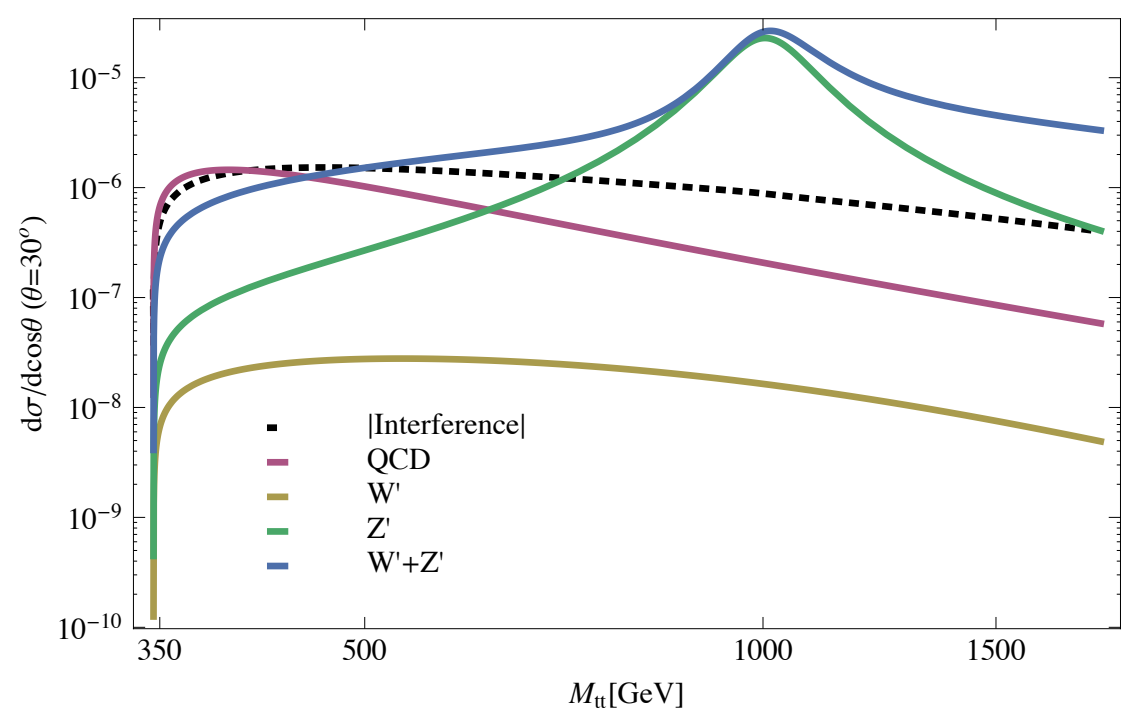

Figure 14: Individual contributions to $d_{R} \bar{d}_{R} \rightarrow t \bar{t}$ at a CM scattering angle of $30^{\circ}$. We plot the magnitude of the interference term. The $Z^{\prime}$ contribution dominates the subprocess in the $1 \mathrm{TeV}$ region. The parton distributions emphasize the low $\hat{s}$ region. 
show a prominent broad peak at the $Z^{\prime}$ mass [55] and an excess of events compared to the SM, as illustrated in Fig. 15. The golden signal of dileptons in the usual $Z^{\prime}$ searches will be difficult to utilize for the ALRM $Z^{\prime}$ due to its small leptonic branching fraction.

\subsubsection{W' signatures}

The detection of the $W^{\prime}$ boson with decays to $t \bar{d}, \bar{t} d$ will provide the definitive evidence for the extra $S U^{\prime}(2)$ symmetry. The subprocess for $W^{\prime}$ production is $d+g \rightarrow t+W^{\prime}$. The cross sections for $W^{\prime}$ production in the ALRM are shown in Fig. 15. The energy dependence of the $t W^{\prime}$ process is shown in Fig. 16. This new physics contribution to inclusive $t \bar{t}$ production is about $5 \%$ of the SM cross section.

The search for a $W^{\prime}$ at the LHC has been discussed in Refs. [51] [56], [57]. The strategy for the determination of the chiral couplings of a generic $W^{\prime}$ that is produced as an $s$-channel resonance and decays to $t \bar{b}$ was demonstrated in Ref. [57], but this process does not exist for the $W^{\prime}$ in the ALRM. A test of the right-handed nature of the $W^{\prime}$ of the ALRM must be made in the process $p p \rightarrow t W^{\prime} \rightarrow t(\bar{t} d)$ which is considerably more difficult because of the ambiguity in reconstruction with two tops in the final state.

\subsubsection{Triplet Higgs}

We have assumed a complex triplet Higgs field $\phi^{\prime}\left(T^{\prime}=1, Y^{\prime} / 2=1\right)$ in order to generate a large mass splitting of the $W^{\prime}$ and $Z^{\prime}$ bosons of the ALRM. Thereby, 


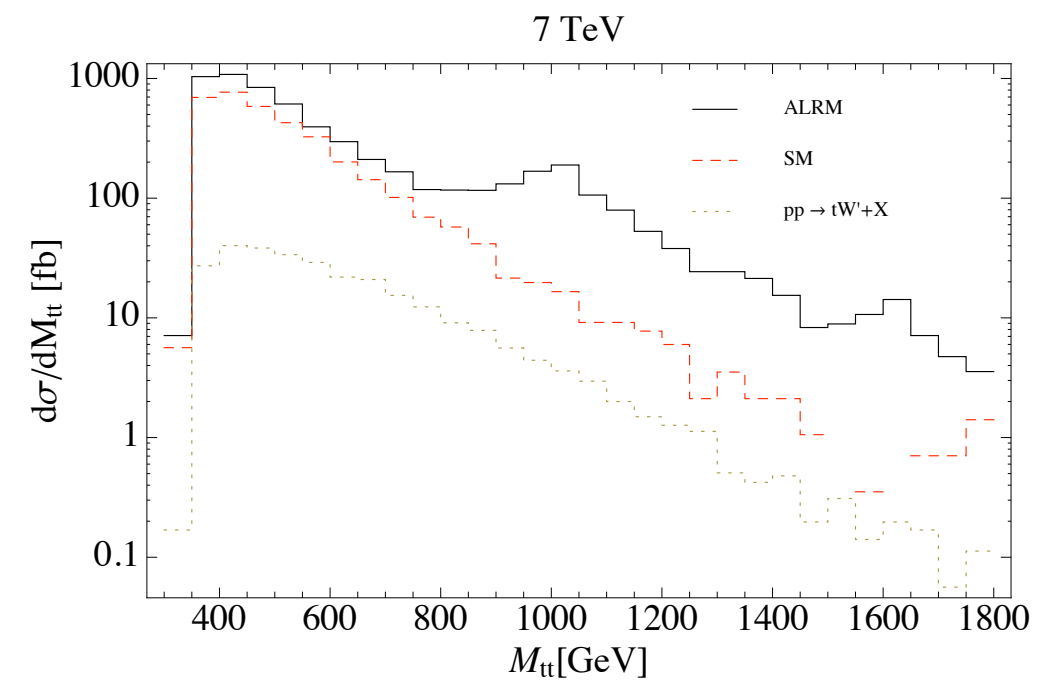

(a) $7 \mathrm{TeV}$ at the LHC

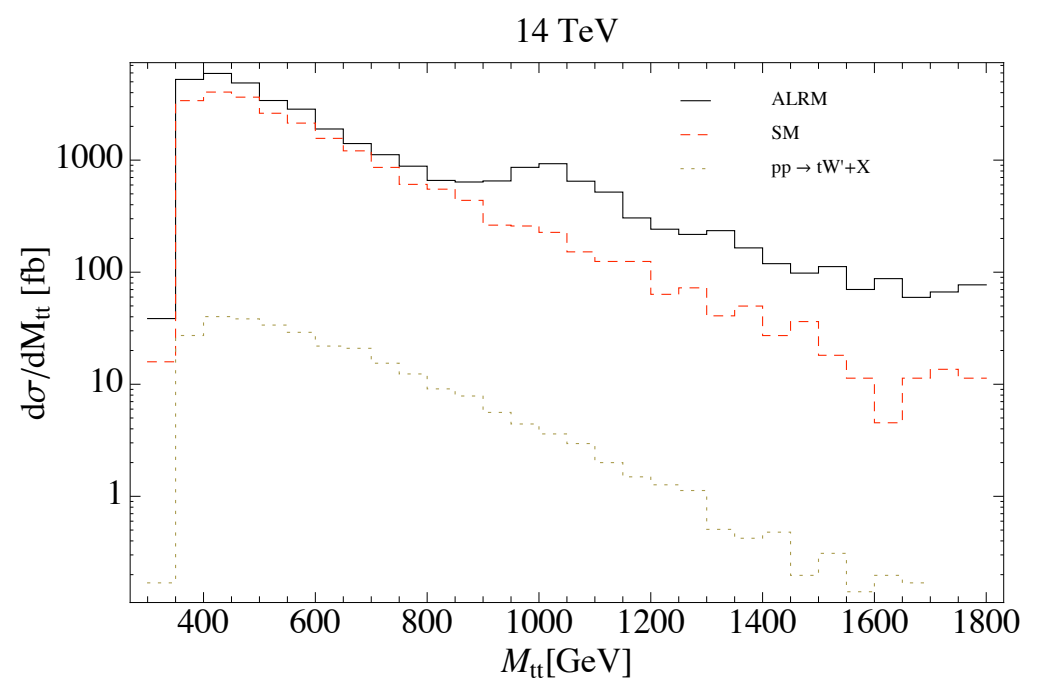

(b) $14 \mathrm{TeV}$ at the LHC

Figure 15: $M_{t \bar{t}}$ distributions at the LHC for $p p \rightarrow t \bar{t}+X$ at 7 and $14 \mathrm{TeV}$. There is a $Z^{\prime}$ resonance peak at $M_{Z^{\prime}} \sim 1 \mathrm{TeV}$ as well as an excess in higher mass bins for the ALRM as compared to the SM. 


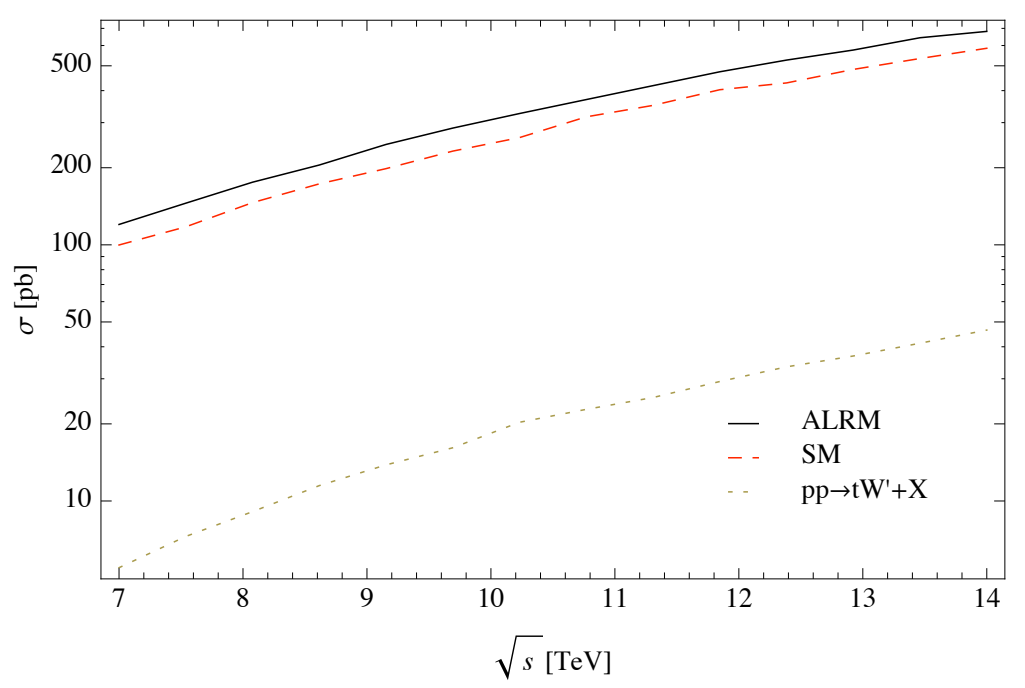

Figure 16: $\sigma(p p \rightarrow t \bar{t}+X)[\mathrm{pb}]$ vs. $\sqrt{s}[\mathrm{TeV}]$ for the SM and ALRM at the LHC. Also plotted is the new physics cross-section for $p p \rightarrow t W^{\prime}+X$.

the $W^{\prime}$ can be sufficiently light to explain the asymmetry data, while the $Z^{\prime}$ can be sufficiently heavy to escape the $Z^{\prime}$ mass bounds in the $t \bar{t}$ channel at the Tevatron. The $\phi^{\prime}$ has no SM couplings. The physical states of the Higgs triplet are $\left(\chi^{++}, \chi^{+}, \chi^{0}\right)$. The detection of Higgs triplet states in hadronic collisions has been discussed in the literature (see Ref e.g. [58]), but not for a model like the ALRM where the triplet Higgs states only couple to pairs of W' or Z' bosons. The tell-tale signature for a complex Higgs triplet is its doubly charged members $\chi^{++}$and $\chi^{--}$, which will decay to $W^{\prime} W^{\prime}$. However, their production will require the $W^{\prime} W^{\prime}$ fusion process, which will be suppressed by the high $W^{\prime}$ mass and by the presence of two top quarks from the $d \rightarrow t W^{\prime}$ transitions in the primary fusion process. SLHC energies are likely to 
be necessary to study this channel.

\subsection{Conclusions}

The measurement by the CDF collaboration of the forward-backward asymmetry $\left(A_{F B}\right)$ of $t \bar{t}$ pairs exceeds the SM expectation and could be smoking gun evidence of new physics. A number of theoretical scenarios have been considered to explain the anomalous asymmetry. Our proposal is a new gauge symmetry $U^{\prime}(1) \times S U^{\prime}(2) \times$ $S U(2)$, which is broken to the SM by the neutral member of a complex Higgs triplet field. We show that the predicted $A_{F B}$ in this model tracks the data in a $A_{F B}$ vs. $M_{t \bar{t}}$ plot, with a $A_{F B}$ that grows with $M_{t \bar{t}}$ over the CDF range. The characteristics of the CDF measurements of $A_{F B}$, the $M_{t \bar{t}}$ distribution, the rapidty difference, and the total $t \bar{t}$ cross section can be reproduced. The preferred masses are $M_{W^{\prime}}=700 \mathrm{GeV}$ and $M_{Z^{\prime}}=1 \mathrm{TeV}$. The $W^{\prime}$ has a right handed $(t, d)$ coupling and the $Z^{\prime}$ is coupled mainly to $t \bar{t}$ and $d \bar{d}$. The discovery of the $Z^{\prime}$ with large $t \bar{t}$ and $d \bar{d}$ couplings at the LHC should be possible with early LHC data and will definitively test our proposed $S U^{\prime}(2)$ gauge symmetry. A recent paper [59] has investigated the implications of flavor violation in the ALRM with maximal mixing of $(t, d)_{R}$ and $(u, b)_{R}$ doublets. They find consistency with the constraints of flavor physics 


\section{Chapter 4}

\section{Looking for parity nonconservation from strong interactions beyond}

\section{QCD}

\subsection{Introduction}

Quantum Chromodynamics (QCD), the gauge field theory describing the strong interactions of colored quarks and gluons in the Standard Model[60], has been extraordinarily successful in describing physics in both non-perturbative and perturbative regimes. Using the positivity of the Euclidean path integrand for Yang-Mills theory, Vafa and Witten proved that QCD does not spontaneously break parity or CP if $\bar{\theta}=061 . \mathrm{CP}$ conservation in the strong interactions is necessitated by the extreme smallness of experimental upper bounds on the neutron electric dipole moment ${ }^{\mathrm{a}}$. It has been suggested that heavy ion collisions may form metastable phases which

\footnotetext{
${ }^{a}$ There are terms in the QCD Lagrangian that violate the charge-parity $(\mathrm{CP})$ symmetry. Mechanisms have been proposed to solve this strong CP problem, of which the Peccei-Quinn mechanism is the most compelling [62].
} 
allow for parity nonconservation [63]. An induced charge separation along the angular momentum vector of the collision would create an electric dipole moment of the hot gluon matter. There are ongoing searches by the STAR collaboration 64] at the Relativistic Heavy Ion Collider (RHIC) to establish such an effect. Parity-violating effects can also be induced by topological solutions in QCD.

There have been many new physics models proposed to explain the large forwardbackward asymmetry, $A_{F B}$, in top quark pair production seen at the Tevatron[2]. For example, recent works have shown that an axial gluon[46] 25] 65] [66] can provide an explanation for the $A_{F B}$ measurement. For recent reviews of the many new physics models, see e.g. Ref.[46] [34, 67, 68]. However, $A_{F B}$ is a test of chargeconjugation (at tree-level) and not of parity conservation. Instead, one can look at the longitudinal polarization of the top-quark, which is a quantity solely determined by parity nonconservation that can be measured in collider experiments. A model that can lead to observable parity nonconservation is the $s$-channel exchange of a spin-1 $X$-gluon with both vector and axial-vector couplings to quarks [25] [69], which we will use as an illustrative example in this Letter. The importance of the measurements of the longitudinal top-quark polarization has also be noted by other authors[70, 171, 72, 44] [73]. At all orders in perturbation theory, QCD leads to zero longitudinal polarization, and SM electroweak contributions should at most be at the few percent level. Thus, the longitudinal top polarization is free of QCD theory ambiguities, unlike the case for the forward-backward asymmetry or the transverse component of the top polarization, both of which have QCD contributions. 


\subsection{X-gluon Model}

Let $A_{1}$ and $A_{2}$ be non-abelian gauge fields associated with the gauge group product, $S U_{1}(3) \times S U_{2}(3)$. The full symmetry is broken by a bi-fundamental Higgs field $\Phi$ with a vev of the form $\langle\Phi\rangle=V 1$. The surviving gauge symmetry is the vectorial $S U_{V}(3)$. Since $T_{2}|0\rangle=-T_{1}|0\rangle$, when the generators act upon the vev state, the massive $X$-gluon composition is

$$
X=\left(g_{1} A_{1}-g_{2} A_{2}\right) / \sqrt{g_{1}^{2}+g_{2}^{2}},
$$

which has been normalized. The other orthogonal combination is the unbroken massless gluon field,

$$
G=\left(g_{2} A_{1}+g_{1} A_{2}\right) / \sqrt{g_{1}^{2}+g_{2}^{2}} .
$$

$\underline{X \text {-gluon couplings to quarks }}$ The couplings to the generators are

$$
\begin{aligned}
g_{1} A_{1} T_{1}+g_{2} A_{2} T_{2}= & \frac{1}{2} g_{1} g_{2} / g_{X} G\left(T_{1}+T_{2}\right) \\
& +\frac{1}{2} g_{X} X\left(g_{1}^{2} T_{1}-g_{2}^{2} T_{2}\right) .
\end{aligned}
$$

A further simplification gives

$$
\begin{array}{r}
g_{s} G\left(T_{1}+T_{2}\right)+\frac{1}{4}\left(g_{1}^{2}-g_{2}^{2}\right) / g_{X} X\left(T_{1}+T_{2}\right) \\
+g_{X} X\left(T_{1}-T_{2}\right) .
\end{array}
$$

where we define $g_{X}=\frac{1}{2} \sqrt{g_{1}^{2}+g_{2}^{2}}$ and $g_{s}=\frac{1}{2} g_{1} g_{2} / g_{X}$. We set $T_{1}$ to act on $L$ chiral fields and $T_{2}$ on $R$ such that $T_{1}+T_{2}$ acts only on the vectorial current, and $T_{1}-T_{2}$ 
on the axial-vectorial current: $\mathbf{T}_{1}+\mathbf{T}_{2} \longrightarrow \bar{q} \mathbf{T} \gamma^{\mu} q, \mathbf{T}_{1}-\mathbf{T}_{2} \longrightarrow-\bar{q} \mathbf{T} \gamma^{\mu} \gamma_{5} q$. The $X$-gluon interaction Lagrangian is

$$
\mathbf{X} \cdot \bar{q} \mathbf{T} \gamma^{\mu}\left(g_{V}^{q}+g_{A}^{q} \gamma_{5}\right) q
$$

with $t \in q$ and $g_{V}^{2}=g_{A}^{2}-g_{s}^{2}$. This relationship of the couplings is modified if one considers higher dimension operators 65

$$
\begin{aligned}
\mathcal{L} & \supset \Lambda^{-2}\left[\lambda_{Q}^{2}\left(\bar{Q}_{L} \Phi\right) i \not D\left(\phi^{\dagger} Q\right)+\lambda_{U}^{2}\left(\bar{U}_{R} \Phi^{\dagger}\right) i \not D\left(\phi U_{R}\right)\right. \\
& \left.+\quad \lambda_{D}^{2}\left(\bar{D}_{R} \Phi^{\dagger}\right) i \not D\left(\phi D_{R}\right)\right]
\end{aligned}
$$

The vev of the bi-fundamental Higgs $\phi$ allows the left-handed gauge field to act upon the right handed quark, and vice versa such that $A_{1}$ acts on $\mathbf{T}_{\mathbf{1}}+y_{U \mid D} \mathbf{T}_{\mathbf{2}}$ and $A_{2}$ acts on $\mathbf{T}_{\mathbf{2}}+x \mathbf{T}_{\mathbf{1}}$, where $x=\lambda_{Q}^{2} V^{2} / \Lambda^{2}$ and $y_{U \mid D}=\lambda_{U \mid D}^{2} V^{2} / \Lambda^{2}$. We also have

$$
\mathbf{T}_{\mathbf{1}} \pm \mathbf{T}_{\mathbf{2}} \Rightarrow(1 \pm x) \mathbf{T}_{\mathbf{1}} \pm(1 \pm y) \mathbf{T}_{\mathbf{2}}
$$

The kinetic derivative piece $i \not \partial$ is increased by $1+x$ for $Q$ and $1+y_{U \mid D}$ for $U$ or $D$ respectively. After renormalizing the kinetic pieces, we have

$$
\begin{aligned}
g_{A}^{q} & =-\frac{g_{X}}{2}\left(\frac{1-x}{1+x}+\frac{1-y_{q}}{1+y_{q}}\right) \\
g_{V}^{q} & =\frac{g_{1}^{2}-g_{2}^{2}}{4 g_{X}}+\frac{g_{X}}{2}\left(\frac{1-x}{1+x}-\frac{1-y_{q}}{1+y_{q}}\right) .
\end{aligned}
$$

The restrictions on the couplings noted above disappear when higher dimension operators are included.

$\underline{q \bar{q} \rightarrow t \bar{t}}$ The helicity amplitudes for the subprocess $q \bar{q} \rightarrow t \bar{t}$, shown in Fig, 17 , are 
given in Table 8, where $\theta$ is the CM scattering angle, $\beta^{2}=1-4 m_{t}^{2} / \hat{s}$, and

$$
\begin{aligned}
G_{I, V} & =\frac{g_{s}^{2}}{\hat{s}}+\frac{g_{I}^{q} g_{V}^{t}}{\hat{s}-m_{X}^{2}+i m_{X} \Gamma_{X}} \\
G_{I, A} & =\frac{g_{I}^{q} g_{A}^{t}}{\hat{s}-m_{X}^{2}+i m_{X} \Gamma_{X}}
\end{aligned}
$$

The $G_{I, V(A)}$ are functions of $\hat{s}$, the square of the subprocess center-of-mass energy, and carry two subscripts; The first refers to initial quark chiralities, $I=L$ or $R$ where we define $g_{L}=\frac{1}{2}\left(g_{V}-g_{A}\right), g_{R}=\frac{1}{2}\left(g_{V}+g_{A}\right)$. The massless condition on initial quarks simplifies the calculation with couplings in this basis. The second subscript refers to the vectorial or axial-vectorial nature of the top quark couplings, which is more efficient in dealing with massive states.
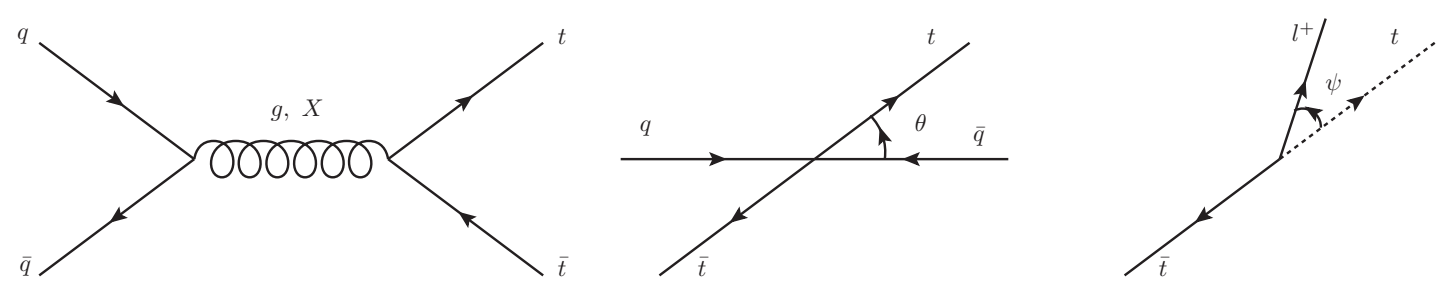

Figure 17: Feynman diagram of the $s$-channel exchanges of the gluon and $X$-gluon in the $q \bar{q} \rightarrow t \bar{t}$ subprocess and the definitions of $\theta$ and $\psi$. The dotted line for $t$ denotes a boost into the $t$ rest frame. 
Table 8: Helicity amplitudes for $q \bar{q} \rightarrow t \bar{t}$

\begin{tabular}{|c||c|c|}
\hline \multirow{2}{*}{ Final State Polarizations } & \multicolumn{2}{c|}{ Initial State Polarizations } \\
\hline \hline-- & -+ & +- \\
\hline++ & $G_{L, V} 2 \sqrt{\hat{s}} m_{t} \sin \theta$ & $G_{R, V} 2 \sqrt{\hat{s}} m_{t} \sin \theta$ \\
\hline-+ & $-G_{L, V} 2 \sqrt{\hat{s}} m_{t} \sin \theta$ & $-G_{R, V} 2 \sqrt{\hat{s}} m_{t} \sin \theta$ \\
\hline+- & $-\left(G_{L, V}-\beta G_{L, A}\right) \hat{s}(1+\cos \theta)$ & $\left(G_{R, V}-\beta G_{R, A}\right) \hat{s}(1-\cos \theta)$ \\
\hline & $\left(G_{L, V}+\beta G_{L, A}\right) \hat{s}(1-\cos \theta)$ & $-\left(G_{R, V}+\beta G_{R, A}\right) \hat{s}(1+\cos \theta)$ \\
\hline
\end{tabular}

We define $\tilde{\sigma}(\theta)=\sum|\mathcal{M}|^{2}(q \bar{q} \rightarrow t \bar{t})$, which is the subprocess differential crosssection modulo an overall factor ${ }^{\mathrm{b}}$. Then, we have

$$
\begin{aligned}
\tilde{\sigma}(\theta) & =\left[A^{+}(-\beta)+A^{+}(\beta)\right]\left(1+\cos ^{2} \theta\right) \\
& -2\left[A^{-}(\beta)-A^{-}(-\beta)\right] \cos \theta \\
& +2\left(1-\beta^{2}\right) A^{+}(0) \sin ^{2} \theta
\end{aligned}
$$

where

$$
A^{ \pm}(\beta)=\hat{s}^{2}\left(\left|G_{L, V}+\beta G_{L, A}\right|^{2} \pm\left|G_{R, V}+\beta G_{R, A}\right|^{2}\right)
$$

Note that the second line of Eq4.10 gives rise to the forward-backward asymmetry. Since the $s$-channel gluon and $X$-gluon amplitudes have identical color structure, the polarization and asymmetry predictions are independent of the parton distribution functions.

For the $7 \mathrm{TeV}$ run of the LHC (LHC7), the analysis is complicated by subprocesses that involve gluons as partons. We will consider these effects in future work.

$$
\mathrm{b} \frac{d \hat{\sigma}}{d \cos \theta}=\frac{\beta}{576 \pi \hat{s}} \tilde{\sigma}(\theta) .
$$


However, by a selective choice of the rapidity region that emphasizes the $q \bar{q} \rightarrow t \bar{t}$ subprocess, it may be possible to probe parity and $C$ nonconservation at the LHC[73], as well as at the Tevatron.

\section{Longitudinal Polarization of Top}

For our purposes, we will consider the leading-order production of top quarks. To all orders of QCD, the top quarks produced are unpolarized. However, in $X$-gluon models, the chiral structure gives rise to partially polarized tops. The longitudinal polarization of the top is described by

$$
P_{\|}=\frac{\sum\left[\left|\left(h_{q}, h_{\bar{q}},+, h_{\bar{t}}\right)\right|^{2}-\left|\left(h_{q}, h_{\bar{q}},-, h_{\bar{t}}\right)\right|^{2}\right]}{\sum\left[\left|\left(h_{q}, h_{\bar{q}},+, h_{\bar{t}}\right)\right|^{2}+\left|\left(h_{q}, h_{\bar{q}},-, h_{\bar{t}}\right)\right|^{2}\right]}=\frac{\tilde{\sigma}_{\|}(\theta)}{\tilde{\sigma}(\theta)}
$$

where the sum is over helicities and $\tilde{\sigma}(\theta)$ is given by Eq. 4.10. The numerator can be simplified as

$$
\begin{aligned}
\tilde{\sigma}_{\|}(\theta) & =\left[A^{+}(\beta)-A^{+}(-\beta)\right]\left(1+\cos ^{2} \theta\right) \\
& -2\left[A^{-}(-\beta)+A^{-}(\beta)\right] \cos \theta
\end{aligned}
$$

The antilepton $\ell^{+}$from the top decay has an angular distribution given by $(1+$ $\left.P_{\|} \cos \psi\right)$, where $\psi$ is defined in Eq.4.16. We obtain a similar expression for $\bar{t}$ by exchanging $h_{t} \leftrightarrow h_{\bar{t}}$. The corresponding angular distribution of $\ell^{-}$is $1-\bar{P}_{\|} \cos \bar{\psi}$. From CP-nonconservation, $P_{\|}=-\bar{P}_{\|}$, so that the angular distributions of $\ell^{ \pm}$are symmetric under $C P$. 
The angle $\psi$ is defined as the angle between the $\ell^{+}$and the negative $\bar{t}$ momentum in a boosted frame in which the $t$ is at rest. The Lorentz boost to the $t \bar{t} \mathrm{CM}$ frame gives

$$
E_{t \bar{t}}\left(e^{+}\right)=E_{t}\left(e^{+}\right)(1+\beta \cos \psi)\left(\frac{1}{2} M_{t \bar{t}} / m_{t}\right)
$$

so that

$$
\begin{gathered}
\left(2 m_{t} / M_{t \bar{t}}\right) p_{\ell^{+}} \cdot\left(p_{t}+p_{\bar{t}}\right) / M_{t \bar{t}}=p_{\ell^{+}} \cdot p_{t}(1+\beta \cos \psi) / m_{t} \\
\cos \psi=\left[\frac{2 m_{t}^{2}}{M_{t \bar{t}}^{2}}\left(\frac{p_{\ell^{+}} \cdot p_{\bar{t}}}{p_{\ell^{+}} \cdot p_{t}}+1\right)-1\right] / \sqrt{1-\frac{4 m_{t}^{2}}{M_{t \bar{t}}^{2}}}
\end{gathered}
$$

The expression above makes use of covariant 4-dot-products and can be evaluated in

any frame. Here, we assume all momenta can be reconstructed in the experiment. $\bar{\psi}$ is obtained from Eq4.16 with substitutions $\psi \rightarrow \bar{\psi}, \ell^{+} \rightarrow \ell^{-}$, and $t \leftrightarrow \bar{t}$.

\subsection{Phenomenology}

For our illustrations, we adopt the parameters $\left|g_{A}\right|=g_{s} / 3, M_{X}=420 \mathrm{GeV}$, and $\Gamma_{X}=42 \mathrm{GeV}$ of the $A_{F B}$ model of Ref. [65], but we allow for the possibility of a vector coupling as well, which leads to parity nonconservation. For simplicity, we consider maximal parity nonconservation scenarios, which we denote as $V \pm A$. The new physics contribution to the $M_{t \bar{t}}$ distribution is found to be similar in the $V \pm A$ cases to that for $A$-only in Ref.[65], as shown in Fig.18. We do not take into account smearing due to the experimental $M_{t \bar{t}}$ resolution. We show the dependence of the $A_{F B}$ on top-pair invariant mass $M_{t \bar{t}}$ in Fig. 19(a). 


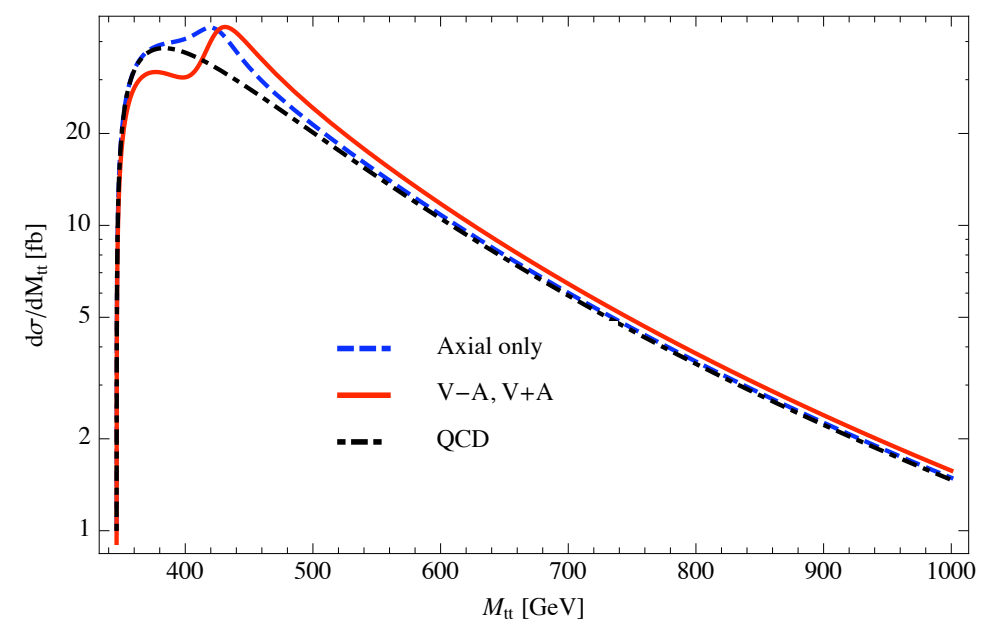

Figure 18: The new physics (NP) contributions to $d \sigma / d M_{t \bar{t}}$ vs. $M_{t \bar{t}}$ in the $X$-gluon model at the Tevatron.

We show the dependence of $P_{\|}$on $M_{t \bar{t}}$ in Fig. 19(b). The polarization goes to zero near $M_{t \bar{t}}=M_{X} \cdot P_{\|}$is largest for $\theta=\pi$. Zero polarization is predicted for a purely axial or vector coupling.

\subsection{Conclusions}

For top-quarks produced via the subprocess $q \bar{q} \rightarrow t \bar{t}$, the longitudinal t-quark polarization $\left(P_{\|}\right)$vanishes in QCD. As a new physics illustration, we have shown that the $s$-channel exchange of a massive $X$-gluon with chiral quark couplings gives rise to a substantial $P_{\|}$. Our study emphasizes the low-energy phenomenology and its parity nonconservation. Additional fermions are needed for UV completion and to cancel 


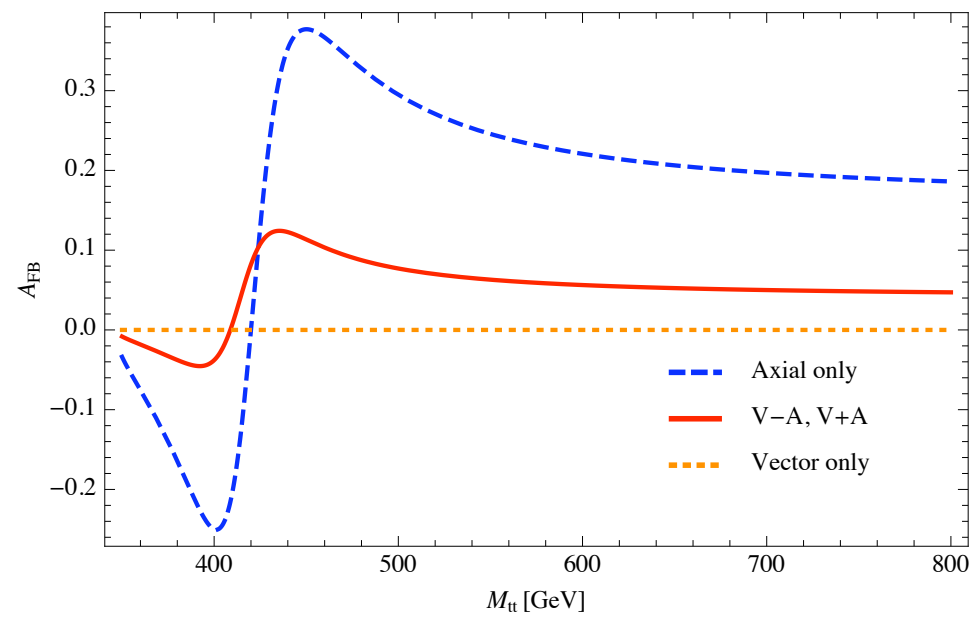

(a) $A_{F B}$ vs. $M_{t \bar{t}}$ for $V \pm A$ (solid), Axial-only (dashed), and Vectoronly (dotted) couplings.

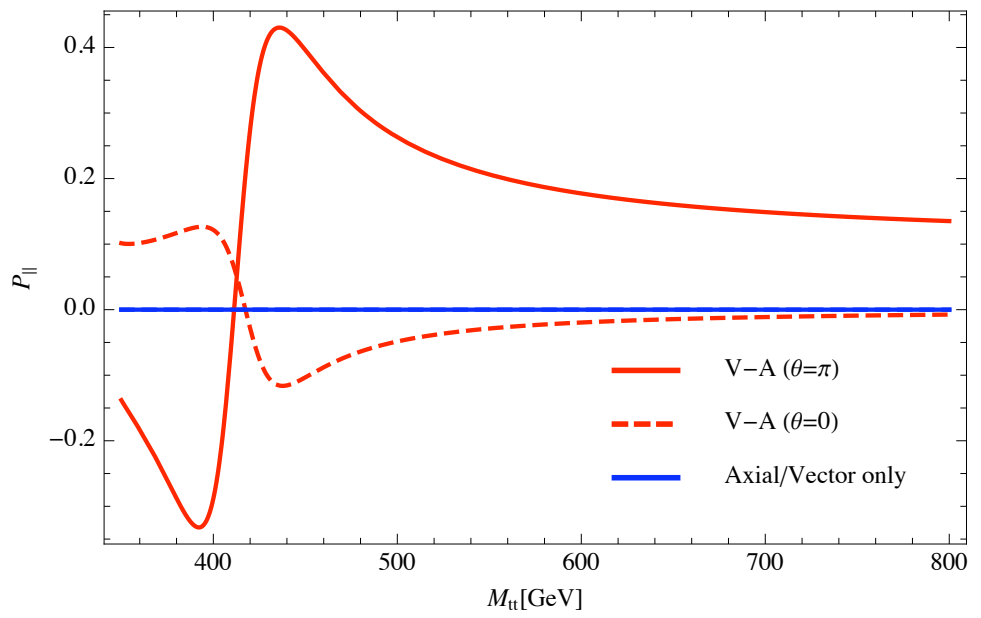

(b) $P_{\|}$vs. $M_{t \bar{t}}$ for $\theta=0$ (dashed) and $\pi$ (solid). $P_{\|}$for $V+A$ is opposite in sign to $P_{\|}$for $V-A$.

Figure 19: $A_{F B}$ and $P_{\|}$vs. $M_{t \bar{t}}$ for $M_{X}=420 \mathrm{GeV}, \Gamma_{X}=42 \mathrm{GeV}$, and $\left|g_{A}\right|=g_{s} / 3$. 
anomalies. The longitudinal polarization is a measurement of $\sigma_{t} \cdot\left(\mathbf{p}_{t}-\mathbf{p}_{\bar{t}}\right) /\left|\mathbf{p}_{t}-\mathbf{p}_{\bar{t}}\right|$ in the $t$ rest frame. Being odd in spatial parity, it is expected to be zero in all orders of perturbative QCD. Thus measurements of $P_{\|}$in $t \bar{t}$ events arising from the $q \bar{q} \rightarrow t \bar{t}$ subprocess at the Tevatron and LHC could prove to be of fundamental importance in finding parity nonconservation in strong interactions beyond QCD. 


\section{Chapter 5}

\section{Stops and MET: the shape of things to come}

\subsection{Introduction}

In the search for physics beyond the Standard Model (SM), the top sector holds unique significance. As the top quark has the largest Yukawa coupling to the Higgs, it contributes one of the largest loop corrections to the Higgs mass, exacerbating the Higgs naturalness problem. To avoid a large degree of tuning, we therefore expect a top partner [74, 75] that is not too much heavier than the top itself, and can be considerably lighter than most other new physics states.

In models of softly-broken supersymmetry (SUSY), this expectation is reinforced by the connection between electroweak symmetry breaking and soft SUSY breaking. In any such model, one can write an expression that relates the mass of the Standard Model $Z$ boson to a linear combination of soft-breaking masses, together with the supersymmetric Higgsino mass parameter $\mu$. This implies either that the soft-breaking mass parameters are not too far above the electroweak scale, or that 
the underlying high energy theory enforces relations among parameters that lead to cancellations in the effective low energy theory. However the latter option is itself strongly constrained by the renormalization group (RG) running of the soft-breaking SUSY parameters and SM parameters, which imply a complicated mapping from the high scale theory to the effective theory probed by experiments. The largest RG effects are related to the largest couplings, and again the top sector has unique importance. This implies that one or both of the stop squarks, the scalar superpartners of the top quark, are expected to be relatively light.

In R-parity conserving SUSY the stop is not a good dark matter candidate, so we will neglect the possibility that the lightest stop is also the lightest superpartner (LSP), and assume that the actual LSP is a weakly interacting particle such as a gaugino. It is quite possible, however, that the lightest stop $\left(\tilde{t}_{1}\right)$ is the the next-tolightest superpartner (NLSP). Because of $R$-parity and charge conservation, stops are produced in pairs in hadronic collisons. Once produced, a stop will decay to the LSP plus SM particles, a decay that can be two body, three body, four body, or even more, depending upon the mass spectrum of the other superpartners whose off-shell couplings connect the stop to the LSP.

In the LHC era, null results from searches for extensions to the Standard Model have excluded new strongly interacting particles with masses that in some cases exceed a TeV [76, 77]. While the LHC experimental searches have been inclusive, the resulting mass limits vary according to the production cross section and decay properties of the new particles. In particular squark mass limits derived from LHC 
experiments often assume four flavors of degenerate squarks, with an additional two-fold degeneracy between the squark partners of left-handed and right-handed quarks. These limits obviously do not apply to a single light stop. Both ATLAS and CMS have begun to constrain models in which pair-produced gluinos decay via stop-top pairs [77, 78], but of course signals in this mode depend on the gluinos being kinematically accessible. Direct stop production has been constrained in the special case that both stops decay to a top and a neutralino, and the neutralino then decays to a gravitino and a $Z$; in this topology ATLAS excludes stops up to $240-330 \mathrm{GeV}$ (depending on the neutralino mass) using $2.05 \mathrm{fb}^{-1}[79]$.

In many models, including SUSY models (on which we focus our attentions), the top partner decays directly to a top and an undetected weakly-interacting particle $(i . e . \tilde{t} \rightarrow t \chi)$, leading to a final state with missing transverse energy $\left(\mathbb{E}_{T}\right)$. Our analysis will focus exclusively on this possibility, which is the most generic. If there is a sufficiently light chargino then the decay $\tilde{t} \rightarrow b \chi^{+}$becomes important, and we will consider this important case in a sequel to this report. Other special cases require more specialized consideration; for example light sleptons enhance stop decays with multilepton final states. For stops lighter than the top, decays could proceed either through an off-shell top (a possibility we will consider in this work), an off-shell chargino, or through a flavor-changing decay $\tilde{t} \rightarrow c \chi[80$, 81, 82, 83, 84]. The possibility of such very light stops is already constrained by Tevatron searches [85, 86], but covering all of the remaining parameter space at the LHC is challenging [87, 88, 89]. 
For stop pairs decaying via $\tilde{t} \rightarrow t \chi$ the current leading technique looks for excesses in $t \bar{t}+\mathbb{E}_{T}$ with the top pair decaying into (semi-)leptonic final states [90, 91]. A recent study of the LHC reach suggests that semi-leptonic analysis could extend the bounds to $750-800 \mathrm{GeV}$ with $20 \mathrm{fb}^{-1}$, assuming that the lightest superpartner particle (LSP) is much less massive than the stop [92]. For heavier stops, the existing searches can be improved by using boosted top-tagging [93, 94, 95]. However, as this requires a large splitting between the mass of the stop and the top+LSP pair, it is ineffective when the stop $p_{T}$ is below $\sim 200 \mathrm{GeV}$. In Refs. [96, 97], it was estimated that, if updated to $1 \mathrm{fb}^{-1}$, LHC searches [98, 99] combined with previous searches at the Tevatron [100, 101, 102, 103, 104] could exclude direct stop pair production decaying to light gravitinos for stop masses up to $180 \mathrm{GeV}$. New results from stop searches with the full 2011 datasets are expected soon from ATLAS and CMS. Clearly, this is a search of considerable interest to the experimental and theoretical community; as a result, during the completion of this paper, we became aware of two additional theoretical groups working on the improving stop sensitivity at the LHC [105, 106].

Many theorists have considered the possibility that a light stop may be nearly degenerate in mass with the sum of the masses of its decay products. Some have even proposed that "degenerate" stops are a natural result of well-motivated SUSY models. For example in Ref. [107] an explicit model was presented with a nearly massless LSP and a lightest stop with mass $188 \mathrm{GeV}$. The literature on degenerate stops has so far assumed that $m_{\tilde{t}}-m_{\chi} \simeq m_{t}$ implies that such particles are invisible to $\mathbb{E}_{T}$-based LHC searches, even if the stops have rather large production rates. 
This implicit no-go theorem is especially strong for stops decaying predominately via $\tilde{t} \rightarrow t \chi$, where the stop pair signal mimics conventional $t \bar{t}$ production. Even away from the degenerate limit, semi-leptonic decay channels have the disadvantage that $\mathbb{E}_{T}$ from the LSPs has to compete with the $\mathbb{E}_{T}$ contributed by neutrinos from top decays.

In this report we dispel this conventional pessimism about LHC detection of degenerate or nearly-degenerate stops, specifically for stops that are at least as heavy as the top quark. We present search techniques that are sensitive to the pair production of top partners decaying into tops and invisible particles, even in the case of exactly degenerate mass spectra. We consider both the semi-leptonic final state (isolated muon or electron plus hadronic jets plus $\mathbb{E}_{T}$ ) and the fully hadronic final state (jets $\left.+\mathbb{E}_{T}\right)$. For the semi-leptonic case we assume a conventional lepton trigger, while for the hadronic final state we assume a four-jet trigger as already implemented by CMS and ATLAS [108].

Our first major observation is that the $\mathbb{E}_{T}$ distribution for stop pair production differs significantly from that of $t \bar{t}$, even in the case where the stops are degenerate. This follows from the fact that $\mathbb{E}_{T}$, despite its calorimeter-centric origins, is a measurement of missing momentum, not missing energy, as well as the fact that stops and tops have a significant decay width. The resulting intrinsic differences in $\mathbb{E}_{T}$ for stops and tops are then magnified by boosted kinematics, taking advantage of the large phase space accessible to stop and top production at the LHC. 
Our second major observation is that even rather small differences in $\mathbb{E}_{T}$ or $\mathbb{E}_{T^{-}}$ related spectra can be detected using a shape analysis. For $\mathbb{E}_{T^{-}}$based observables we show that, in the kinematic regime where signal is enhanced, the distributions of the relevant backgrounds are well-described by simple analytic functions. This background-fitting technique is motivated by the CMS Razor searches [109, 110, 111], which in 2010 and 2011 successfully implemented one and two-dimensional shape analyses into inclusive SUSY searches and a third-generation leptoquark search. The Razor searches are based on the Razor kinematic variables $M_{R}$ and $R$, where $R$ is related to the $\mathbb{E}_{T}$ fraction of the event [112]. Rather than attempt to reproduce the 2D Razor fitting techniques, our analysis focuses on simpler 1D shape analyses. The success of the Razor both validates the realism of our basic approach, and suggests that the application of the CMS Razor to a degenerate stop search would result in equal or greater sensitivity than discussed here.

In Section 5.2, we describe in detail our search strategy, focusing on the missing transverse momentum distribution of stop events, as well as the fitting of background distributions. In Section 5.3, we use the results of the MadGraph, Pythia, and modified PGS4 simulation tools to demonstrate the reach of our technique for hadronic stop searches in the next year of LHC running. Finally, in Section 5.4, we apply the shape analysis technique to a kinematic distribution related to $\mathbb{E}_{T}\left(M_{T}^{W}\right)$ in semi-leptonic top decays. In our conclusions, we present the expected exclusion limit using the combination of these two orthogonal searches; as we will show, using the expected 
luminosity from the LHC in $2012\left(20 \mathrm{fb}^{-1}\right)$ these shape analyses can potentially exclude stops up to $560 \mathrm{GeV}$ when the LSP is very light, and up to $360 \mathrm{GeV}$ when the sum of the top and LSP masses are degenerate with the stop. This constitutes a significant improvement over the current and projected bounds from the standard stop searches.

\section{$5.2 \quad \mathbb{E}_{T}$ and $\mathbb{E}_{T}$-related Methods}

As discussed above, the SUSY scenario we wish to consider is one in which the stop is considerably lighter than the other squarks (and gluino) and decays directly to a top and the LSP, which for concreteness we take to be a neutralino. In particular, we will consider the simplified model [113] of a single light stop squark $\left(\tilde{t}_{1}\right.$, henceforth $\tilde{t})$ which decays to a top and a neutralino. Since the stop is a colored scalar, its production is dominated by QCD processes and so is only very weakly sensitive to the details of the rest of the superpartner spectrum

The rate and kinematics of the $\tilde{t} \rightarrow t \chi$ process are determined by two parameters: the stop mass, $m_{\tilde{t}}$, and the neutralino mass, $m_{\chi}$. Once the stop decays, and the neutralino escapes the detector the only visible states in the signal events are the decay products of the tops. The only remaining indication of stop production is the missing transverse energy carried away by the LSPs. In Figure 20 we show the production cross section at NLO for LHC with $\sqrt{s}=8 \mathrm{TeV}$. All other superpartner masses are set to $1 \mathrm{TeV}$, except for the neutralino. Clearly, the small production 
cross section for a single stop pair, combined with the lack of multiple observables to distinguish from background makes the search for stops challenging.

However, the presence of intrinsic $\mathbb{E}_{T}$ is a handle that allows signal to be distinguished from backgrounds. The existence of $\mathbb{E}_{T}$ in stop events will not only affect the number of events with large $\mathbb{E}_{T}$ but also the distribution of these events. Furthermore, the background distribution of $\mathbb{E}_{T}$ can be well modeled using simple analytic functions, which can, in many cases be measured in high statistics control regions. Using the shape of the $\mathbb{E}_{T}$ distribution provides a powerful tool to distinguish signal and background, as we outline below.

\section{Background $\notin_{T}$ shapes}

Since the signal contains the decay products of two top quarks, and intrinsic $\mathbb{E}_{T}$, the largest SM backgrounds will come from $t \bar{t}$, QCD multi-jet production and $W+$ jets. Which of these processes dominates depends on the range of $\mathbb{E}_{T}$ and the mode of top decay. In order to limit the source of non-LSP $\mathbb{E}_{T}$ (which would dilute the signal), we consider only fully hadronic top decay, for which our analysis applies a lepton veto; and semi-leptonic top decays, for which we require exactly one isolated lepton.

For the case of fully hadronic tops (the full analysis of which is described in Section 5.3), there are two major sources of $\mathbb{E}_{T}$ in top background events. The first is from detector mis-measurement of top events where both $W$ 's decay hadronically. The second case - which dominates at large $\mathbb{E}_{T}$ - is due to one or both of the $W$ 's 


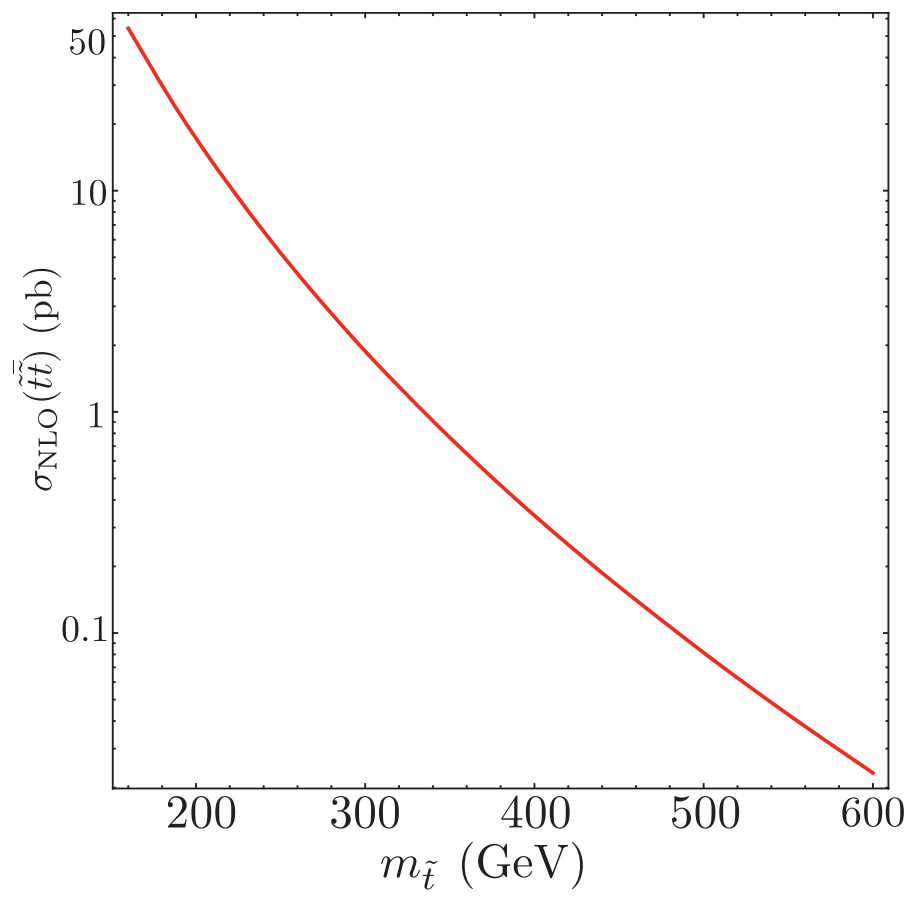

Figure 20: Stop pair production at $\sqrt{s}=8 \mathrm{TeV}$, calculated at NLO using Prospino [4].

decaying into a $\tau$ which in turn decays hadronically. In this case the $\nu_{\tau}$ present in the top decay provides an intrinsic source of $\mathrm{SM} \mathbb{E}_{T}$. Other sources of intrinsic $\mathbb{E}_{T}$ in hadronic SM events arise include neutrinos from heavy flavor decays, and events where one or both of the $W^{\prime}$ 's decay leptonically and all charged leptons in the decay are lost, either due to acceptances or detector effects. For the QCD background the dominant source of $\mathbb{E}_{T}$ is mis-measurement of the jets.

For semi-leptonic top decays (detailed in Section 5.4), the sources of $\mathbb{E}_{T}$ are similar, although as we now require one charged lepton there will be more neutrinos 
(either from leptonic decays of the $W$ or leptonic decays of a $\tau$ ). The $W+$ jets process is a relevant (but subdominant) background for the semileptonic analysis and here the $\mathbb{E}_{T}$ comes from the leptonic decay of the $W$. The main QCD contribution is from jets faking leptons, but the rate for this is low. In the background events with a leptonically decaying $W$, the transverse mass of the lepton and the $\mathbb{E}_{T}$ should lie below the $W$ mass; there is however a tail above the $W$ mass generated by events with a leptonic $W$ and a hadronic $\tau$. As we will show in the next section, this arrangement of background $\mathbb{E}_{T}$ allows for a significant increase in signal over background by combining $\mathbb{E}_{T}$ with other kinematic information into a transverse mass variable $M_{T}^{W}$.

\section{$\mathbb{E}_{T}$ and $M_{T}^{W}$ distributions in signal}

By looking in the hadronic channel with a lepton veto, the separation between events with intrinsic $\mathbb{E}_{T}$ (signal), and those with other sources of $\mathbb{E}_{T}$ (background), can be maximized. One might expect that the stop signal missing transverse energy would also be very small, especially when the masses of the LSP and stop are such that $\Delta \equiv m_{\tilde{t}}-\left(m_{t}+m_{\chi}\right) \approx 0$, making separation difficult. However, it is important to remember that the name 'missing transverse energy' is a misnomer. It is not the transverse energy that is measured - rather the detectors record transverse momentum. In the rest frame of the parent stop, the momentum of the LSP is

$$
Q=\frac{\sqrt{\left[m_{\tilde{t}}^{2}-\left(m_{t}+m_{\chi}\right)^{2}\right]\left[m_{\tilde{t}}^{2}-\left(m_{t}-m_{\chi}\right)^{2}\right]}}{2 m_{\tilde{t}}} .
$$


For small splitting the missing momentum scales as $Q \approx \sqrt{2 \mu \Delta}$ if $\Delta \ll m_{\chi}$ and $Q \approx \sqrt{\Delta\left(2 m_{\chi}+\Delta\right)}$ if $\Delta \sim m_{\chi} \ll m_{t}$ (here $\mu$ is the reduced mass of the neutralinotop system). In all but the last case the scale of the missing momentum is enhanced above that of the small mass splitting, proportional only to the square root of the small mass scale.

Even in the limit where the stop is completely degenerate with the top-neutralino system $(\Delta=0)$, the decay will proceed through the stop (or top, though this possibility was neglected in the Monte Carlo methods used in this paper) being off-shell by an amount comparable to the width $\Gamma$. In this limit, where we assume the decay is still prompt, $\Delta$ should be replaced with $\Gamma$ in the above expressions. Thus, for stops produced $\gtrsim 5 \mathrm{GeV}$ off shell and $m_{\chi} \gtrsim 50 \mathrm{GeV}$, we expect the LSPs to carry $\sim 20 \mathrm{GeV}$ of momentum each, in the rest frame of the top.

The intrinsic $\mathbb{E}_{T}$ of the event is obtained from the vector sum of the LSP transeverse momenta in the lab frame. Each stop is not generically at rest in the lab frame, and is boosted with respect to the center-of-mass frame of the partonic collision. The presence of ISR activity also provides a transverse boost, and causes the tops and neutralinos resulting from the stop decays to not be back-to-back, increasing the $\mathbb{E}_{T}$. Taking all these effects into account, we expect a harder distribution of $\mathbb{E}_{T}$ in stop pair events than in $t \bar{t}$ events, even for degenerate stops . This is confirmed by explicit simulation, as we will show. Note that our detailed simulations with MadGraph and Pythia use the matrix element for stop pair production plus an extra jet to more accurately model the effect on $\mathbb{E}_{T}$ of the stop pair recoiling against an extra energetic 
jet.

For the case of semi-leptonic top decays, the background, as outlined above, also contains irreducible sources of $\mathbb{E}_{T}$. However, in these cases there is a $\mathbb{E}_{T}$-related variable that distinguishes signal and background: the transverse mass $M_{T}^{W}$ defined below. Though the visible decay products are identical in signal and background, we can try to distinguish the two by considering the difference between the invisible components. For signal, the $\mathbb{E}_{T}$ consists of two LSPs and a neutrino, while for background, it comes predominantly from a single neutrino, which partners with the visible lepton to form a $W$ boson. If we assume that all events come from SM $t \bar{t}$ events, and thus that the neutrino $p_{T}$ is equal to the observed $\mathbb{E}_{T}$, then we can attempt to reconstruct the $z$-component (up to a two-fold ambiguity) of the neutrino momentum, using the $W$-mass as a constraint:

$$
p_{z}^{\nu}=\frac{p_{z}^{\ell}\left(M_{W}^{2}+2 \vec{p}_{T}^{\ell} \cdot \vec{E}_{T}\right) \pm E_{\ell} \sqrt{\left(M_{W}^{2}+2 \vec{p}_{T}^{\ell} \cdot \vec{E}_{T}\right)^{2}-4\left(p_{T}^{\ell}\right)^{2} \not_{T}^{2}}}{2 p_{T}^{\ell}} .
$$

Clearly, if the missing energy is either inaccurately measured or not due to a $W$ induced neutrino, then this reconstruction will fail. One indication of such a failure would be if the quantity in the square root can be negative. Defining

$$
\left(M_{T}^{W}\right)^{2} \equiv 2\left(p_{T}^{\ell} \mathbb{E}_{T}-\vec{p}_{T}^{\ell} \cdot \vec{E}_{T}\right)
$$

we can improve the signal over background ratio by restricting ourselves to the region $M_{T}^{W}>M_{W}$. This improvement arises because only mis-measurement and hadronic taus can drive $M_{T}^{W}$ into this regime for background, while for signal, the vector 
sum of two neutralinos and the neutrino can easily result in $\mathbb{E}_{T}$ that satisfies this constraint, even without mis-measurement.

\section{Shape analyses}

Experimental analyses, particularly at hadron colliders, have tended to shy away from modeling the shape of MET distributions. In final states dominated by jets, there is the complicated phenomenon of jet mis-measurement, or more generally the nonlinear response of the calorimetry used for the standard calorimeter-based reconstruction of MET. However, the ATLAS and CMS experiments have already demonstrated the ability to understand MET distributions in a variety of complex final states, and to simulate MET including the contributions to MET from imperfect detector response and reconstruction [114, 115]. Already in the 2010 LHC run, the Razor analysis at CMS demonstrated the usefulness of modeling MET-based observables for inclusive SUSY searches [116], and a similar approach was applied in the 2011 run to a Razor search for relatively light third-generation leptoquarks [111]. The latter is especially relevant to the search for light stops, since it involved $b$-tagging and was optimized for lighter particles producing weaker MET signals. These successful shape analyses in jet-dominated final states in LHC data validate that the basic approach pursued in this report can, with suitable modifications, be mapped into successful searches.

For semi-leptonic final states there is an even stronger track record of successful modeling of MET-based observables. In particular, the spectacularly precise determinations of the $W$ boson mass by the CDF and D0 experiments were based on 
modeling of the $M_{T}^{W}$ distribution in large lepton-triggered data sets [117]. For stop searches we require much less precision in the determination of the shape, and we are interested exclusively in modeling the tail above the Jacobian peak, rather than the peak itself.

For our study we have relied on simulated samples, with events generated by MadGraph5 [118], showered and hadronized by Pythia6 [119, and physics objects reconstructed using PGS [120]. The use of MadGraph allows us to simulate both SUSY signals and the $t \bar{t}$ background with extra partonic jets included in the matrix element. This adds essential realism both in that initial state radiation (ISR) effects are important when simulating degenerate stops, and because our baseline selection relies on counting jets. PGS has been shown to give reasonably accurate results for MET and other basic observables for the case of SUSY signals [121, 122] and, by extension, $t \bar{t}$ as long as one does not probe too far out in the tails of distributions.

Accurate simulation of QCD multijet backgrounds and the MET associated with them is a more serious challenge, both because of the difficulty of generating samples with sufficient Monte Carlo statistics, and trusting features of such samples in a toy detector simulation after making very hard cuts. For our analysis we generated the equivalent of approximately $2 \mathrm{fb}^{-1}$ of QCD multijets. We used the loosest of several different baseline selections (all requiring $b$-jets tagged to varying degrees of strictness) that seemed to give roughly comparable sensitivity, with the idea that this makes our background modeling more reliable. While we have some confidence that our results agree at least qualitatively with distributions obtained from LHC 

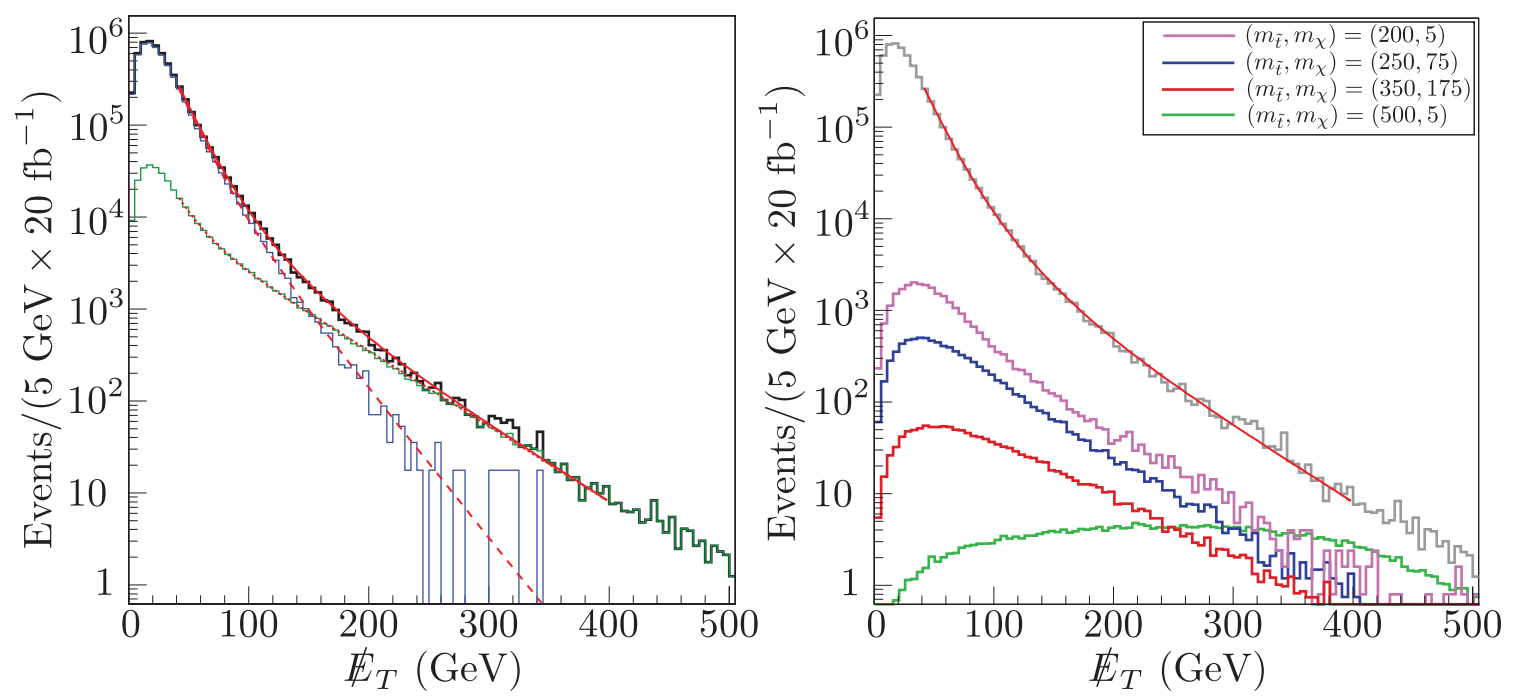

Figure 21: Left: Differential distribution of events for $20 \mathrm{fb}^{-1}$ with respect to $\mathbb{E}_{T}$ of QCD (blue) and $t \bar{t}$ (green), and the total background (black) passing the hadronic trigger. The analytic fits to Eq. (5.4) using the parameters in Table 9 are shown in red for QCD (dashed), $t \bar{t}$ (dotted) and their sum (solid). Right: Differential distribution of events corresponding to $20 \mathrm{fb}^{-1}$ with respect to $\mathbb{E}_{T}$ for signal $\tilde{t} \tilde{t} \rightarrow t \bar{t} \chi \chi$ passing the hadronic trigger for a range of stop and LSP masses $\left(m_{\tilde{t}}, m_{\chi}\right)$.

data, our simulated background samples should be considered as placeholders for data control samples in a real LHC analysis.

For this study we simulated the three largest backgrounds: QCD multijets, $t \bar{t}+$ jets, and $W+$ jets, but neglect the smaller contributions from $Z / \gamma^{*}+$ jets, dibosons, single top, and $t \bar{t}+Z$.

Figure 21 (left) shows the background MET distributions that we obtain after our hadronic baseline selection (detailed in Section 5.3). QCD multijets dominates for MET values below about $150 \mathrm{GeV}$, while $t \bar{t}$ dominates above. $W+$ jets and other backgrounds were found to have a negligible effect on the MET shape above about 
$40 \mathrm{GeV}$. Above $40 \mathrm{GeV}$, both the QCD and $t \bar{t}$ backgrounds have MET distributions with a simple shape. Both shapes are well-described by the sum of two exponentials, a feature reminiscent of the kinematic shapes in the Razor analyses. The results of a fit (from RooFit [123]) in the MET range between 40 and $400 \mathrm{GeV}$ are shown in red in the figure. The MET distributions of the hadronic signals from light stops also have simple shapes, as illustrated in Figure 2 (right). As expected, while the signals suffer from lower cross sections compared to background, for MET exceeding $\sim 100 \mathrm{GeV}$ they start to emerge as significant distortions of the MET shape. For degenerate stops the signal MET shapes have an exponential drop-off that is similar - but not identical - to that of $t \bar{t}$.

One could employ a more traditional "cut and count" approach to the light stop analysis, but it is clear from Figure 21 that such an analysis would be complicated by the variety of different signal shapes and signal MET regions of interest. However, it can serve as a useful cross-check, and so (as we will show), we have performed a simple cut-and-count analysis for comparison to our shape analysis. An intermediate approach is to replace our analytic fits to the background shapes with a coarselybinned analysis of MET yields; however given the simplicity of the background shapes it is not surprising that such a "poor-man's" shape analysis has less sensitivity when compared to the full shape analysis.

For the semileptonic analysis, the variable of interest is $M_{T}^{W}$ rather than $\mathbb{E}_{T}$; specifically, we are interested in the shape of $M_{T}^{W}$ above the mass of the $W$, where 


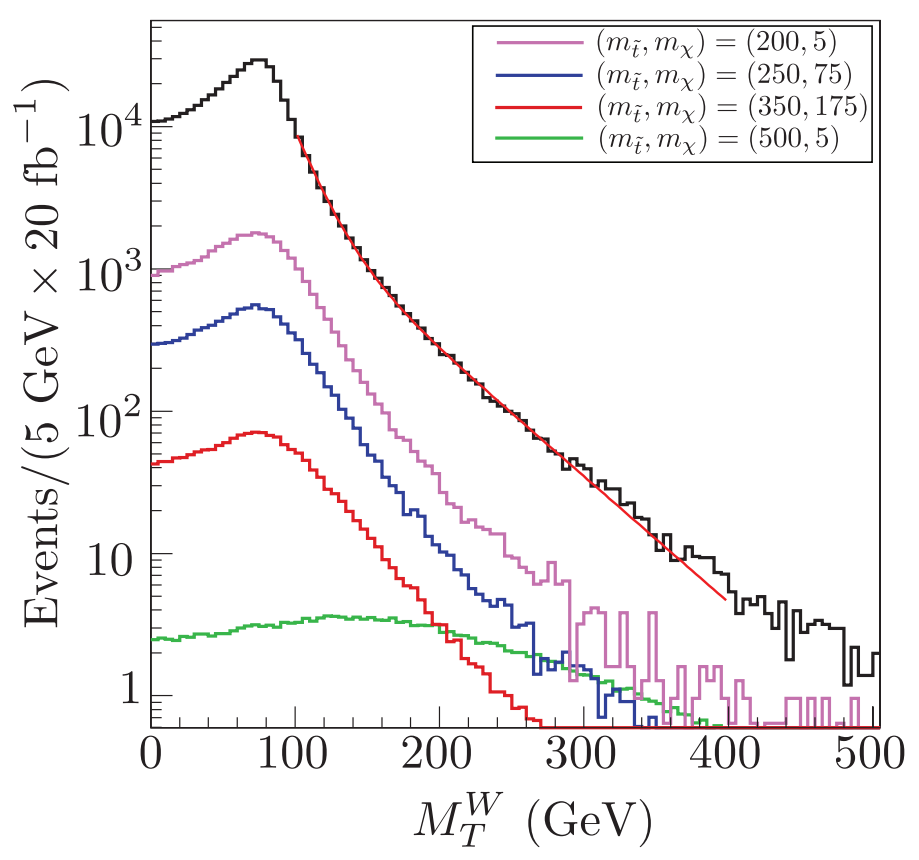

Figure 22: Differential distribution of $t \bar{t}$ events with respect to $M_{T}^{W}$ (black). The analytic fit (Eq. (5.4) using the parameters of Table 10) is shown in red. Also shown are the differential distributions of stop signal events with respect to $M_{T}^{W}$ for a range of stop and LSP masses. The semi-leptonic event selection is described in Section 5.4.

background is reduced. Using a lepton trigger followed by a tight $b$-tag, can significantly reduce $W+$ jets background in this range, leaving only $t \bar{t}$ as the dominant background (the full baseline selection is described in Section 5.4). Using the same event generation as in the hadronic case, we show in Figure 22 the distribution of $t \bar{t}$ background with respect to $M_{T}^{W}$. Above $M_{W}$, this distribution can, like $\mathbb{E}_{T}$, be fit with a pair of exponentials, greatly simplifying the shape analysis. Signal distributions for a representative sample of stop and LSP masses are also shown; as in the 
fully hadronic case, the shapes are sufficiently different to allow discrimination.

\subsection{LHC Search for Hadronic Stops}

In order to look for the effects of stops in the shape of $\mathbb{E}_{T}$ in hadronic events, we must first significantly reduce QCD background. We do this by applying a baseline selection based on an all-hadronic trigger, simplifying those developed by ATLAS and CMS for LHC running. We require at least two jets with $p_{T}>80 \mathrm{GeV}$ and at least two additional jets with $p_{T}>50 \mathrm{GeV}$, with a requirement of $|\eta|<3$ for all jets. Of the jets with $p_{T}$ above $50 \mathrm{GeV}$, two must be tagged as $b$-jets; at least one must pass a "tight" b-tagging requirement, and the second must pass at least the "loose" requirement. Events that contain any electrons with $p_{T}>20 \mathrm{GeV},|\eta|<2.5$ or any muons with $p_{T}>20 \mathrm{GeV},|\eta|<2.1$ are vetoed (for our simulations, taus are treated as jets, thus forming a irreducible background that contains large $\mathbb{E}_{T}$ ).

To calculate the efficiencies with which tops, QCD, and signal stop events pass the trigger, we perform Monte Carlo simulation of the CMS detector by using the following procedure: We use MadGraph5/MadEvent to generate $t \bar{t}$ backgrounds and $\tilde{\tilde{t}} \bar{t}$ signal events, matched to one additional jet. The $\tilde{t} \rightarrow t \chi$ branching ratio is set to 1 , and top decay is handled by Pythia6. The top mass is assumed to be $175 \mathrm{GeV}$. Detector simulation is done by PGS4, modified to more closely match the reported CMS b-tagging efficiencies for both "tight" and "loose" thresholds, as found in Ref. [124]. The top cross section at $\sqrt{s}=8 \mathrm{TeV}$ was calculated to be $226.9 \mathrm{pb}$ using 

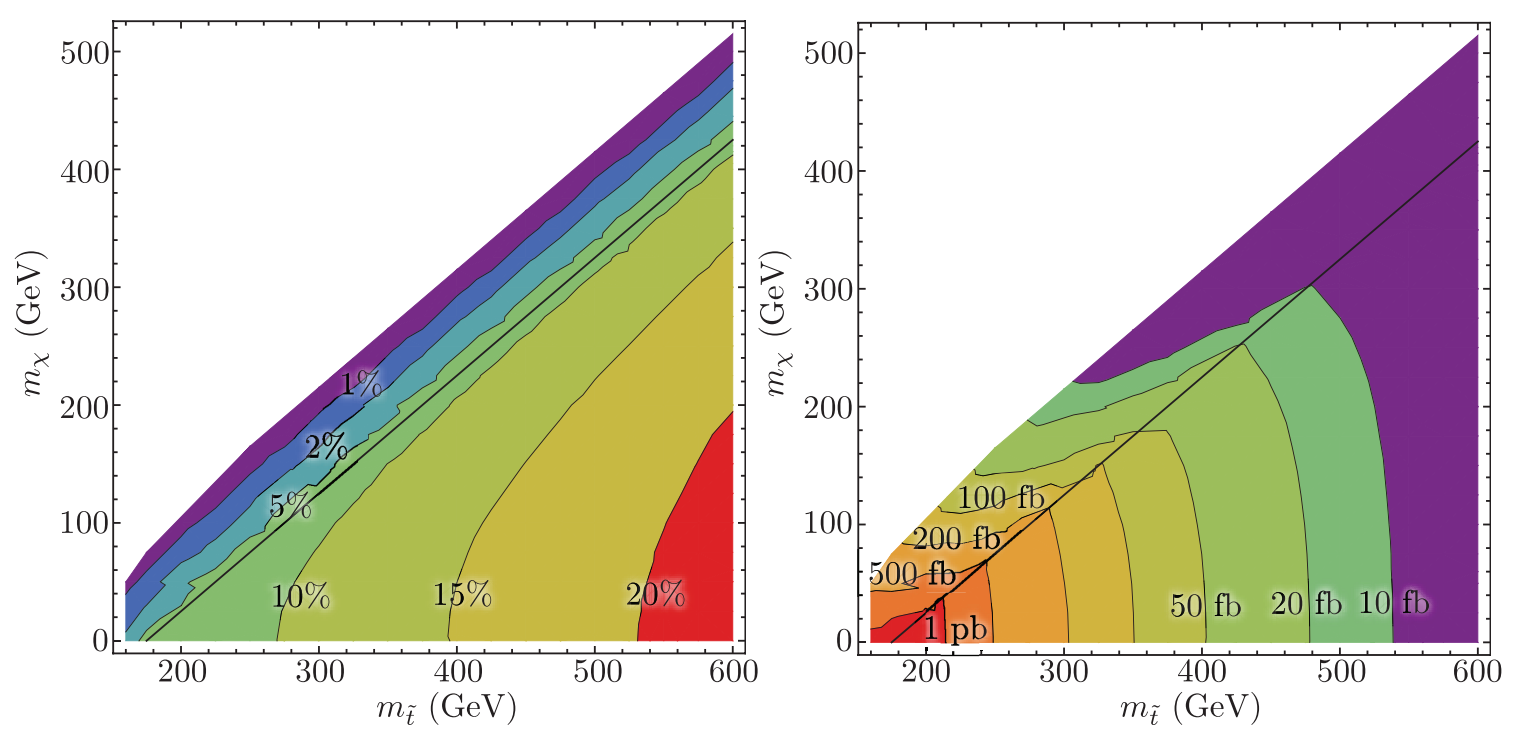

Figure 23: Left: Signal trigger efficiency as a function of stop and LSP masses for hadronic event selection. Right: Signal cross section times trigger efficiencies as a function of stop and LSP masses. Like all such plots in this paper, the contours are extrapolated from a grid of Monte Carlo results with $5-25 \mathrm{GeV}$ spacing in $m_{\tilde{t}}$ and $m_{\chi}$. The degeneracy line $\left(m_{\tilde{t}}=m_{t}+m \chi\right)$ is shown in black.

MCFM at NLO, while the stop cross sections were determined using Prospino [4, and are shown in Figure 20.

Using these simulations we find that the trigger has a $\sim 7 \%$ pass efficiency for background tops, while the stop signal efficiency can vary from 2\%-20\% percent, depending on the mass splitting between the stops and the LSP (see Figure 23). Larger splittings lead to more energetic tops in the decay, and so result in more high- $p_{T}$ jets and a higher trigger efficiency. We generated four-jet QCD background events in MadGraph, and allowed them to hadronize and shower through Pythia6, which produced a higher multiplicity of jets. The QCD total cross section and 
differential rates at LHC7 were compared to ATLAS experimental results [125], and scaled to LHC8 by taking the ratio of Alpgen [126] partonic cross sections at LHC7 and LHC8. After application of our jet trigger selection and $b$-tag requirements, we find that $\sim 265 \mathrm{pb}$ of QCD background remains. However, only $17 \%$ of these events have $\mathbb{E}_{T}$ above $40 \mathrm{GeV}$.

As stated previously, assuming perfect detectors and no contamination from events with leptons (and thus neutrinos), the top and QCD backgrounds should have zero $\mathbb{E}_{T}$. However, this is clearly not an assumption that survives contact with reality. Mismeasurement of jets, mis-tags of electrons and taus, and other experimental effects will all contribute non-zero $\mathbb{E}_{T}$ to the background. As we are limited to publicly available tools in our simulations, we cannot hope to exactly reproduce the $\mathbb{E}_{T}$ distribution in top events which will be observed by CMS and ATLAS. However, our PGS simulation of the detector (using the CMS detector geometry) will be sufficient to demonstrate the general behavior.

In the left panel of Figure 21, we plot the $\mathbb{E}_{T}$ distribution for background events (in $5 \mathrm{GeV}$ bins) passing our trigger selection criteria, using an initial set of 60 million QCD MadGraph/Pythia/PGS events and 27 million top events. Two important features can be easily noticed. First, the $\mathbb{E}_{T}$ background peaks at $\sim 20 \mathrm{GeV}$; this is at or below the intrinsic $\mathbb{E}_{T}$ value of stop events for all mass parameters of interest. Second, past the peak, each background is exponentially falling. We separately fit each background to a sum of two exponentials,

$$
\frac{d \sigma}{d \mathbb{E}_{T}}=A e^{-\alpha \mathbb{E}_{T}}+B e^{-\beta \dot{E}_{T}} .
$$




\begin{tabular}{|c|c|c|}
\hline & QCD & $t \bar{t}$ \\
\hline$\alpha$ & $6.9 \times 10^{-2} \pm 1.56 \times 10^{-3}$ & $6.29 \times 10^{-2} \pm 1.63 \times 10^{-3}$ \\
$\beta$ & $3.77 \times 10^{-2} \pm 1.26 \times 10^{-3}$ & $1.89 \times 10^{-2} \pm 1.57 \times 10^{-4}$ \\
\hline
\end{tabular}

Table 9: Best fit parameters for QCD and $t \bar{t} \mathbb{E}_{T}$ distributions, fit to (Eq. 5.4 for an integrated luminosity of $20 \mathrm{fb}^{-1}$. Note that these errors are correlated with each other and with the normalizations $(A, B)$, which in turn depend on the amount of integrated luminosity considered. See text for details.

Due to limited statistics in the tail, and the complicated structure at low $\mathbb{E}_{T}$, we only use this analytic fit over the range $40 \leq \mathbb{E}_{T} \leq 400 \mathrm{GeV}$. Other choices for the fitting function are possible (such as a Gaussian or Cruijff function, combined with exponentials), and may increase the range over which the background may be modeled. However, this simple choice suffices for our purposes. The corresponding distributions for signal are shown in the right-hand panel of Figure 21 for a range of stop and LSP masses. For each signal point, we generate between 400,000 and one million matched stop pair events using MadGraph/Pythia/PGS. However, we do not attempt an analytic fit for signal. Notice that, for signal, the total $\mathbb{E}_{T}$ peaks at a higher value than the parton-level $\mathbb{E}_{T}$ does. This is due to the addition of jet mismeasurement in addition to the LSP momenta, which serves to increase the average $\mathbb{E}_{T}$ observed.

Our analysis is based entirely on Monte Carlo (MC) simulated samples. As a result, in order to mimic the effects of statistical fluctuations one would expect to see in data, which will affect the precision of the fits, we carry out the fits outlined above on appropriately chosen samples of MC data. For $t \bar{t}$ we can generate in MC the number of events expected after $20 \mathrm{fb}^{-1}$ of $8 \mathrm{TeV}$ running and use this to extract 
the parameters. For QCD we cannot hope to generate sufficient MC, so instead we carry out the fit on the 60 million QCD events that we have. We then use this fit as an input to generate "pseudo-data" appropriate to $20 \mathrm{fb}^{-1}$, and refit to the pseudodata. This approach captures the uncertainty expected in the fit of real data. We show the best-fit slopes, and the associated errors, in Table 9. However, note that there are sizable correlations between these fit parameters that need to be taken in to account when calculating the uncertainty on the fit.

Although we are handicapped by having to rely on MC to determine the shape of the background distributions, the LHC collaborations do not suffer from this restriction, as they are in possession of copious amounts of data. The QCD background to our signal contains two b-tagged jets, mostly a light quark or charm quark faking a $b$-quark, or from real $b$ production. The complementary anti-b-tagged sample (4 jets above our cuts but with no $b$-tags), provides a clean sample of (predominantly) QCD events in which to measure the $\mathbb{E}_{T}$ distribution. However, in order to extrapolate the $\mathbb{E}_{T}$ distribution from this sample to the signal region the $b$ mis-tag rate in QCD samples, as a function of jet $p_{T}$, must be well understood. Through simulation we estimate that if this mis-tag rate is known to $\sim 20 \%$ accuracy, as a function of $p_{T}$, then the effects on the determination of the parameters describing the QCD background are within our present uncertainties. This is encouraging for a data-based analysis. The $t \bar{t}$ background is harder to determine from data alone, but this issue is beyond the scope of our discussion. 


\section{Maximum likelihood method}

In order to estimate the potential for $20 \mathrm{fb}^{-1}$ of LHC8 data to exclude or observe the stop simplified model at a particular parameter point $\left(m_{\tilde{t}}, m_{\chi}\right)$, we must have some measure of the difference between signal and background $\mathbb{E}_{T}$ curves. The measure we employ is hypothesis testing with profiled likelihoods [127. In this approach one calculates likelihoods assuming the observed data is the result of a particular hypothesis, maximizing the likelihoods over "nuisance" parameters, which in our case are the 8 parameters of the fits to the background $\mathbb{E}_{T}$ shapes. We account for the known correlated uncertainties in the fit parameters by introducing Gaussian penalty terms into the definition of the likelihoods.

Since the above procedure requires access to data, we instead ask the question of how well the experiments can expect to do if the data they observe is due to a particular model. There are two natural hypotheses that we can make for what the LHC may see: a) there is no light stop and the only production mechanisms are from the SM, or b) there is a light stop and the production cross section is as predicted in the MSSM To calculate the likelihoods for these two hypotheses, we can take advantage of our background analytic function as well as the shape of the distribution of signal events, determined from MC, to generate pseudo-data which contains within it an equivalent amount of statistical fluctuation as $20 \mathrm{fb}^{-1}$ of actual data. We generate this pseudo-data using the central values of the best fit parameters

\footnotetext{
${ }^{a}$ There is clearly a continuum of possibilities: that there is a light stop and neutralino but the production cross section is different from what is predicted in the MSSM. Carrying out a full scan in the stop production cross section is beyond the reach of this paper.
} 
found in Table 10). We then attempt to fit this pseudo-data to both the SM only hypothesis and the SM+stop hypothesis.

The log likelihood, including the constraint associated with the Gaussian uncertainties on the background fit parameters, $c_{i}$, is given by

$$
\log L\left(c_{i}, \sigma\right)=\sum_{\text {bins }}-\nu\left(c_{i}, \sigma\right)+n+n \log \left(\frac{\nu\left(c_{i}, \sigma\right)}{n}\right)-\frac{1}{2} \sum_{p q}\left(c_{p}-\bar{c}_{p}\right) C_{p q}^{-1}\left(c_{q}-\bar{c}_{q}\right)
$$

where $\nu$ is the predicted number of events in a bin, $n$ is the observed number of events in a bin for a particular set of pseudo-data, $\bar{c}_{i}$ is the central value of the $i^{\text {th }}$ fit parameter, and $C_{i j}$ is the covariance matrix of those fit parameters. The second summation term in Eq.5.5is a constraint in the maximization, coming from assuming the uncertainties in the parameters of the background fit are Gaussian in nature. We allow the eight parameters involved in the background fits (four normalizations and four slopes) to vary within their uncertainties and maximize the log likelihood over these parameters and the signal production cross-section, $\sigma$. That is, for the SM only hypothesis, we maximize $\log L$ over $\hat{c}_{i}$ and $\hat{\sigma}$, and for the SM+stop hypothesis, we fix $\tilde{\sigma}$ to the NLO expectation, $\sigma_{*}$, and maximize $\log L$ over $\tilde{c}_{i}$. Since the pseudo-data was generated under the SM only hypothesis, $\hat{\sigma} \sim 0$ in all cases.

As our test statistic we use twice the difference between these two values,

$$
2 \Delta \log L=2 \log L\left(\hat{c}_{i}, \hat{\sigma}\right)-2 \log L\left(\tilde{c}_{i}, \sigma_{*}\right)
$$

which for clarity we convert into a number of standard deviations $n_{\sigma}=\sqrt{2 \Delta \log L}$. 
This $n_{\sigma}$ measures the incompatibility of the SM+stop versus SM only profiled likelihoods. We repeat this process 200 times to obtain the average sensitivity.

In addition to the profile likelihood method described above we also investigate the sensitivity along the "degeneracy line" $\left(m_{\tilde{t}}-m_{\chi}=m_{t}\right)$ using the CLs method [128, 129]. We do so by generating $10^{4}$ pseudo experiments under both background

only and signal+background hypotheses and then use these pseudo experiments to determine the expected exclusion of signal, for an observation consistent with background. Since the CLs method requires a high statistics sample of pseudo experiments we did not calculate the bounds for stop masses below $\sim 230 \mathrm{GeV}$. For the median expected exclusion we assume that the log likelihood ratio of the observed data falls at the median of the background-only distribution. For the one sigma CLs band we assume the data falls above/below the background median value by one sigma, and similarly for the two sigma band.

\section{Estimated Hadronic Stop Bounds}

Using these statistical methods, in Figure 24 we show the estimated significances extracted from our test statistic for light stop simplified models when the top decays hadronically.

We estimate that for simplified models in which the stop/neutralino mass splitting is large, the LHC experiments can set strong stop mass limits up to $\sim 550 \mathrm{GeV}$. In the case of a very light neutralino the reach is determined simply by the production cross section of the stops, which drops rapidly with the mass (Figure 200, although 
there is some softening of this behavior due to increased efficiency to pass the cuts as the stop mass is increased (Figure 23).

Most interestingly, even along the mass-degeneracy line of $m_{\tilde{t}}-m_{\chi}=m_{t}$, stops of mass as high as $\sim 350 \mathrm{GeV}$ could be excluded with $20 \mathrm{fb}^{-1}$ of $8 \mathrm{TeV}$ data. In fact we find that the sensitivity reach extends above the degeneracy line into regions where the stops decay into off-shell tops.

As an additional cross-check, we perform a simple cut-and-count analysis of the signal parameter points, dividing the $\mathbb{E}_{T}$ range of $40-400 \mathrm{GeV}$ into three regions: our "background" region of 40-100 GeV; and two signal regions; 100-200 GeV and 200-400 GeV. Iterating over 200 pseudo-experiments generating $\mathbb{E}_{T}$ distributions of signal plus background events, we assume that all events in the background region are ascribable to the QCD and top backgrounds. This sets our overall normalization, which we use to predict (using our analytic fit Eq. (5.4)) the number of the background events in our two signal regions. For each pseudo-experiment, we can then calculate the number of signal events $S$ in each signal region as the difference between the observed events $O$ and the predicted value $P$. In Figure 25, we plot the average value of $S / \sqrt{O}$ for both the low- $\mathbb{E}_{T}$ and high- $\mathbb{E}_{T}$ signal regions. Addition of a realistic systematic error to the predicted number of events will reduce the sensitivity of the cut and count method. For a stop mass of $250 \mathrm{GeV}$ and LSP of $5 \mathrm{GeV}$ one has, with zero systematics, $9 \sigma$ sensitivity, while a $5 \%$ systematic reduces this to approximately $2 \sigma$.

The shape analysis we are advocating allows for many of the backgrounds to be 
determined from control regions in data and thus removes many systematic uncertainties associated with theoretical predictions of background 4 . There are systematic uncertainties associated with extrapolation from control regions to signal regions, such as the b-tagging rates discussed above, but we estimate that these are subdominant to the fit uncertainties. We have, with the exception of QCD, fit to simulated data sets that are consistent with what one would expect after luminosity of $20 \mathrm{fb}^{-1}$ and find the errors as shown in Table 9. However, since our analysis is entirely MC based, and it is possible that the real control regions will contain limited statistics, we also investigate how the sensitivity is affected if the errors in our fit parameters are inflated. In particular we consider the situation where the central values for the fit parameters are as shown in Table 9 but the errors are a factor of 3 or 5 times larger. Assuming that the errors from extrapolation are then subdominant to the fit uncertainty, we keep the correlations between the fit parameters as we inflate the errors. With an inflation by 3 the fractional errors in the fit parameters range from a few to $17 \%$ and inflation by 5 has a largest error of $30 \%$, with the largest errors in the normalizations, as expected. The effects of this inflation, for $3 \times$, are shown in Figure 24. An inflation by $5 \times$ degrades the sensitivity as one moves towards degeneracy: along the degeneracy line the $2 \sigma$ exclusion extends to $260 \mathrm{GeV}$. The $2 \sigma$ exclusion for case of light neutralino is not greatly altered from the bound for $3 \times$ inflation.

\footnotetext{
${ }^{\mathrm{b}}$ There still exists a difficult-to-quantify systematic error associated with the choice of functional form the background distributions are fit to. A discussion of these effects is beyond the scope of this paper.
} 
Focusing on the degeneracy line $\left(m_{\tilde{t}}-m_{\chi}=m_{t}\right)$, a region of particular interest and considerable challenge, we apply the CLs method as outlined above. The median expected exclusion, as well as one and two sigma bands, on such a degenerate stopneutralino pair is shown in Figure 26. Using the CLs method, stop masses up to $375 \mathrm{GeV}$ can be excluded at $2 \sigma$ when $m_{\tilde{t}}-m_{\chi}=m_{t}$.

\section{$5.4 \quad$ Study of $M_{T}^{W}$ shapes}

We now turn from stops with fully hadronic decays of top to the semi-leptonic channel, discussed briefly in Section 5.2. In this case the dominant background is $t \bar{t}$. Given that semi-leptonic $t \bar{t}$ decays have an intrinsic source of missing transverse energy from the neutrinos coming from the $W$ decays, $\mathbb{E}_{T}$ offers poorer discrimination between signal and background, as compared to the hadronic case. We therefore focus instead on the transverse mass variable $M_{T}^{W}$ defined in Equation 5.3 . This variable is related to $\mathbb{E}_{T}$, but has the additional feature that SM background $\mathbb{E}_{T}$ from a single leptonic $W$ decay is mostly distributed below the Jacobian peak near the $W$ mass.

Our method follows the hadronic analysis closely. Again assuming stop pair production, each decaying to a top and an LSP, we now look for events where one top decays leptonically, while the other decays hadronically. We select events with exactly one isolated lepton with $p_{T}>20 \mathrm{GeV}$ and $|\eta|<2.1(2.5)$ for muons (electrons), at least one tight $b$-tagged jet, and requiring three or more jets with $p_{T}>30 \mathrm{GeV}$ and 
$|\eta|<3$. The primary background is reduced to $t \bar{t}$, with an acceptance efficiency of $\sim 15 \%$ (including branching ratios). The efficiencies and cross section times efficiencies for the stop/LSP signal points are shown in Figure 27.

Focusing on $M_{T}^{W}$ above $M_{W}$ will improve the discrimination of stops from tops. Applying a shape analysis as was done in the hadronic $\mathbb{E}_{T}$ case will provide even greater advantages. The total SM background distribution for $M_{T}^{W}>85 \mathrm{GeV}$ can again be well fit by the sum of two exponentials:

$$
\frac{d \sigma}{d M_{T}^{W}}=A e^{-\alpha M_{T}^{W}}+B e^{-\beta M_{T}^{W}}
$$

Repeating the search strategy performed in the hadronic analysis, we use RooFit to find the best fit for the parameters in the $M_{T}^{W}$ range of $85-400 \mathrm{GeV}$ (see Table 10 , weighting the top background to the equivalent of $20 \mathrm{fb}^{-1}$ of data. Again, the fit errors reported are highly correlated.

Using this fit and the associated errors, we repeat the profile likelihood analysis described previously, testing the background versus signal plus background hypotheses over 200 background-generated pseudo-experiments for each simplified model point. Our results are shown in Figure 28, for both the full profile likelihood analysis including all errors, and the case of errors inflated by a factor of 3 . The sensitivity is

\begin{tabular}{|c|c|}
\hline & fit to $20 \mathrm{fb}^{-1}$ total SM background \\
\hline$\alpha$ & $6.68 \times 10^{-2} \pm 6.88 \times 10^{-4}$ \\
$\beta$ & $2.01 \times 10^{-2} \pm 3.04 \times 10^{-3}$ \\
\hline
\end{tabular}

Table 10: Best fit slope parameters for background $M_{T}^{W}$ distribution, fit to (Eq. 5.7). Note that the fit errors are correlated with each other and with the normalizations $(A, B)$, which in turn depend on the amount of integrated luminosity considered. 
similar to that obtained for the hadronic analysis. In Figure 29, we perform a crosscheck using the cut-and-count method, with a background bin between $85-150 \mathrm{GeV}$ used for normalization, a low signal bin between $150-250 \mathrm{GeV}$, and a high signal bin between $250-400 \mathrm{GeV}$. As before, this simple analysis both validates and provides motivation for the full shape analysis.

\subsection{Conclusion}

Third generation squarks are an integral part of the supersymmetric solution to the naturalness and hierarchy problems. More generally, the large Yukawa couplings between the top and the Higgs hint at some connection between the third generation and electroweak symmetry breaking. Improving the search techniques for stop squarks (and more generally, top partners) at the LHC is therefore of great theoretical and experimental interest. In this paper, we have demonstrated that a dedicated search for stop pairs in hadronic and semi-leptonic channels has the potential to improve the current limits, especially for mass values such that the stop and the LSP + top quark system are nearly degenerate.

We see that the tranverse momentum in the lab frame produced by the LSPs in stop pair decays is larger than naive expectations. Thus hadronic searches that limit the contribution to $\mathbb{E}_{T}$ from Standard Model neutrinos can provide significant discrimination between signal and background. The most obvious way to access this kinematic information is by modeling the shapes of $\mathbb{E}_{T}$ distributions for the most 
relevant SM backgrounds. As we have shown, such an analysis is capable of excluding stops up to $\sim 250 \mathrm{GeV}$ in the degenerate case, as compared to up to $550 \mathrm{GeV}$ when the LSP is light. However, we expect that other $\mathbb{E}_{T}$-based variables could also serve. For the semi-leptonic stop search, we saw that the most straightforward approach is to model the shape of the transverse mass variable $M_{T}^{W}$, which is related to $\mathbb{E}_{T}$. We found that the projected sensitivity to degenerate stops in the semi-leptonic case also reaches up to $\sim 300 \mathrm{GeV}$, similar to that in the hadronic channel. Finally, since these two channels are independent, we combine these bounds which we show in Figure 30. The resulting exclusion for light neutralinos is $560 \mathrm{GeV}$ and $360 \mathrm{GeV}$ in the degenerate case.

We note that the CMS Razor analyses [112, 109, 110, 111] access the missing transverse momentum of an event through the transverse Razor variable $M_{T}^{R}$ (and through this, the Razor ratio $R$ ). As such, one would expect that Razor inclusive searches could be competitive with a more targeted analysis using the techniques outlined in this report. More generally, our $\mathbb{E}_{T}$ search could be upgraded to a multidimensional shape analysis as used in the Razor. Though, in this theoretical work, the analytic fits for the $\mathbb{E}_{T}$ distributions were drawn from Monte Carlo simulation, the experimental collaborations can use data control samples to model the background shapes. In the real experimental analyses the optimal baseline selections in both the hadronic and semi-leptonic channels could differ from those presented here. Furthermore, we have shown that even if the extraction of the fit parameters from data suffers from considerably more uncertainty than our Monte Carlo based analysis 
the shape-based approach, unlike a cut and count, still has good reach.

Our results support the assertion that it is not possible for stop squarks lighter than $\sim 1 \mathrm{TeV}$ in $R$-parity conserving SUSY to elude LHC searches over the long run. A stop discovery would be at least as fundamentally important as a Higgs discovery, while complete exclusion of stops with mass lighter than a TeV would be a significant blow to our understanding of the connection between supersymmetry and electroweak symmetry breaking. 

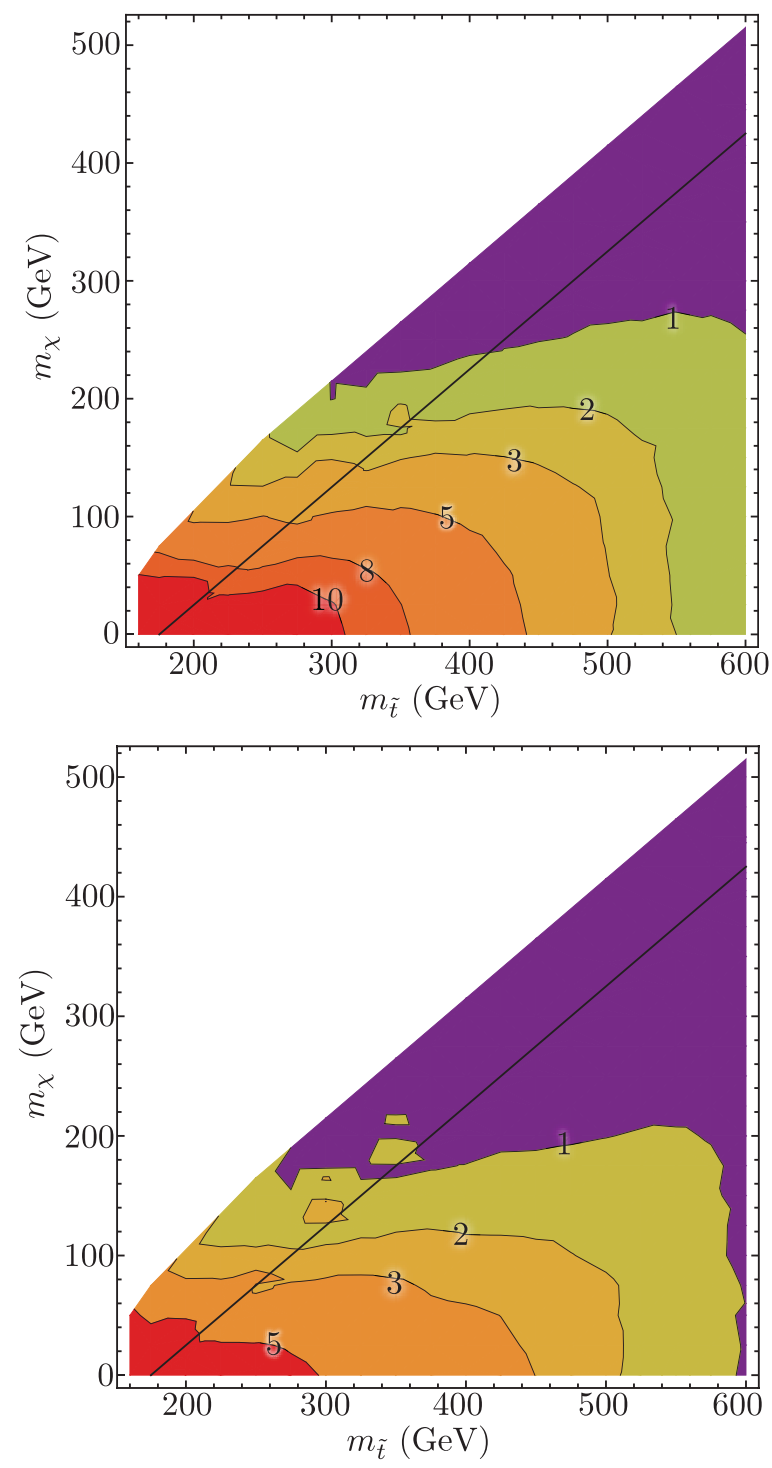

Figure 24: Expected sensitivity, in standard deviations, for the hadronic $\mathbb{E}_{T}$ shape analysis as a function of the stop and LSP masses. The test statistic is computed from 200 pseudo-experiments of $20 \mathrm{fb}^{-1}$. In the left-hand plot the uncertainty on the background $\mathbb{E}_{T}$ shape are as shown in Table 9 and in the right-hand plot these errors have been inflated by a factor of 3 . 

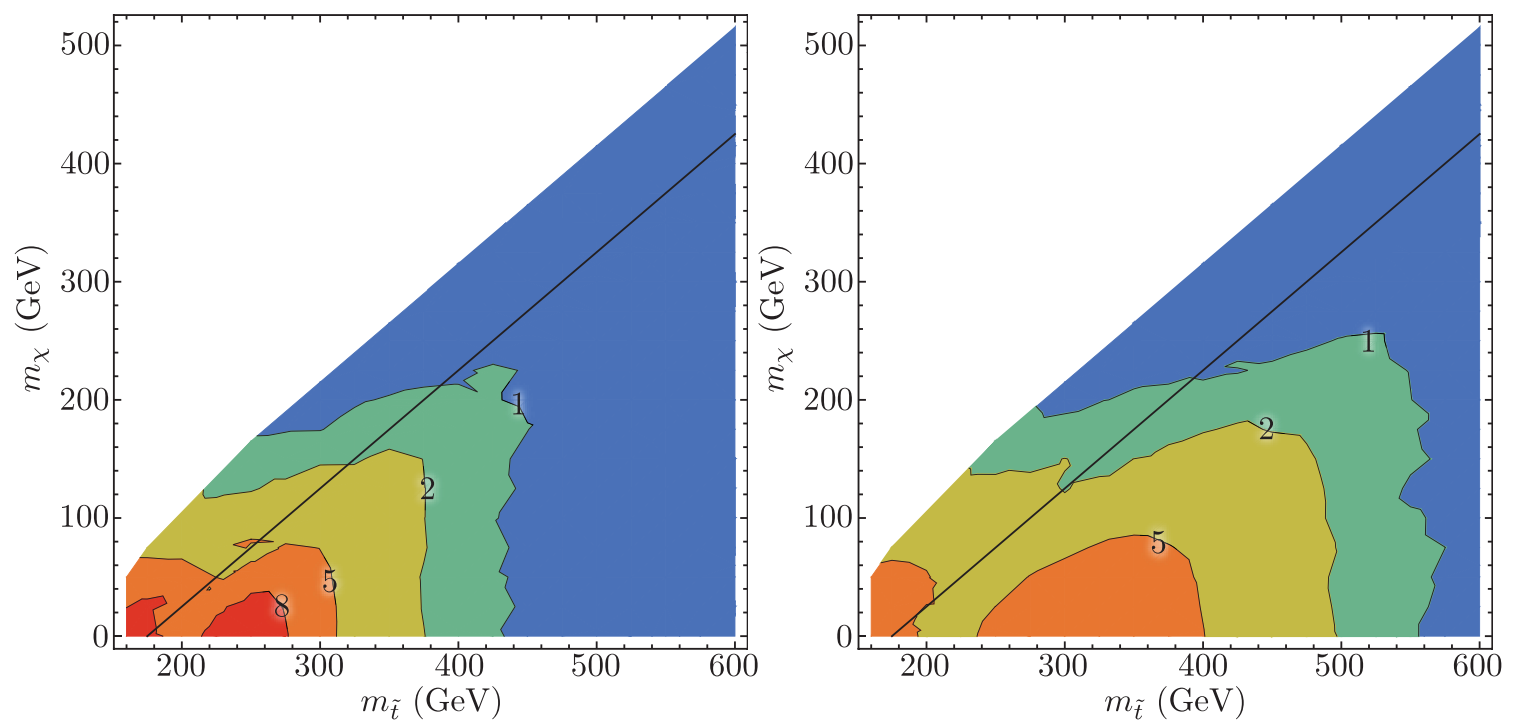

Figure 25: Left: $S / \sqrt{O}$ for $100-200 \mathrm{GeV}$ region of signal plus background $\mathbb{E}_{T}$ distribution. Right: $S / \sqrt{O}$ for $200-400 \mathrm{GeV}$ region. These are computed with an unrealistic assumption of no systematics.

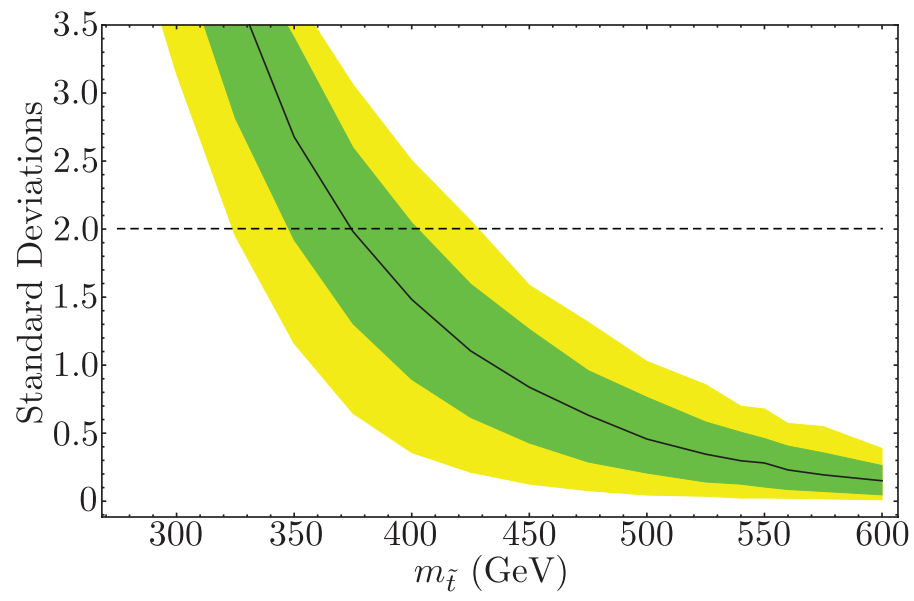

Figure 26: The median expected exclusion, for background only pseudo experiments, on a stop-neutralino simplified model whose masses are related by $m_{\tilde{t}}-m_{\chi}=m_{t}$. 

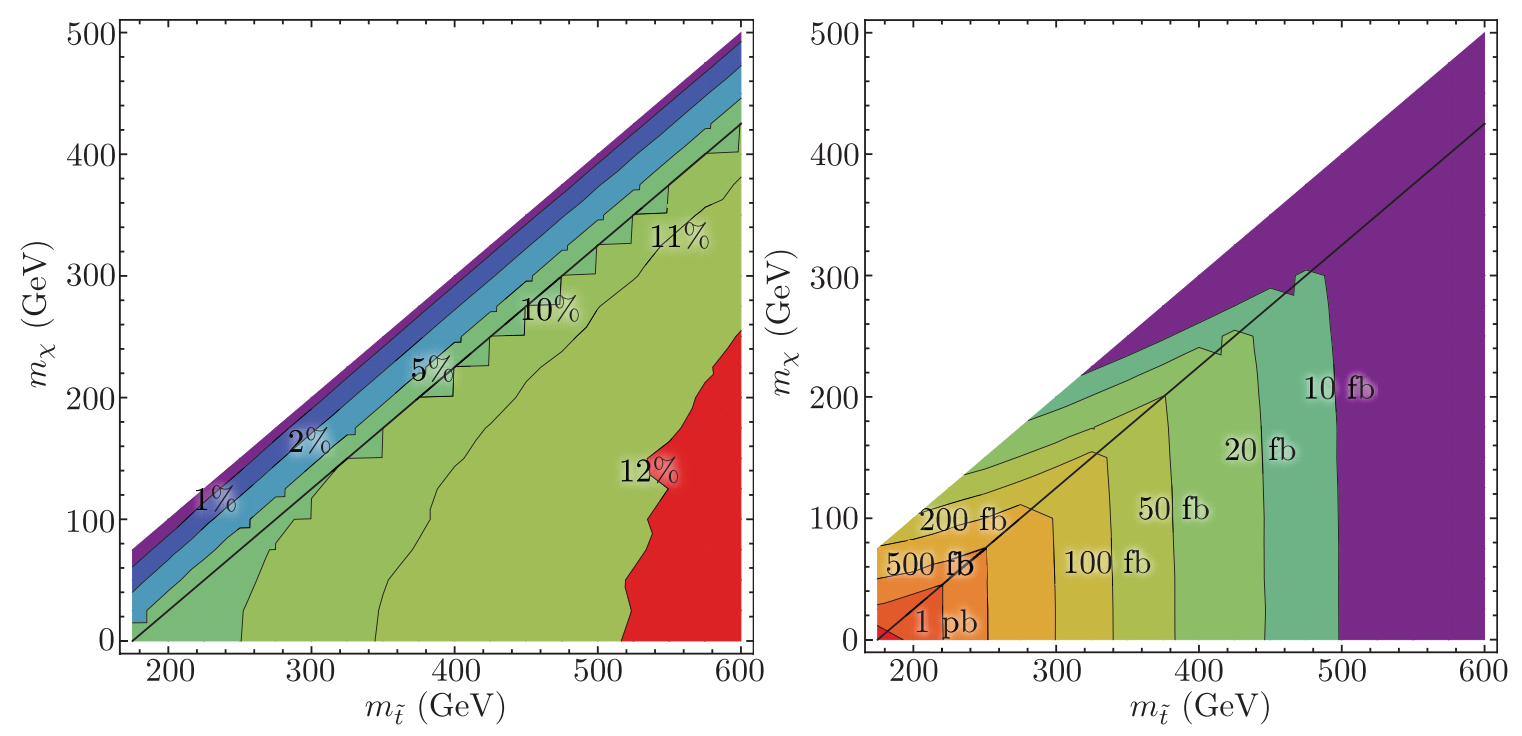

Figure 27: Left: Semi-leptonic trigger efficiency for semi-leptonic events as a function of stop and LSP masses. Right: Cross section times efficiency for the semi-leptonic selection criteria as a function of stop and LSP masses. 

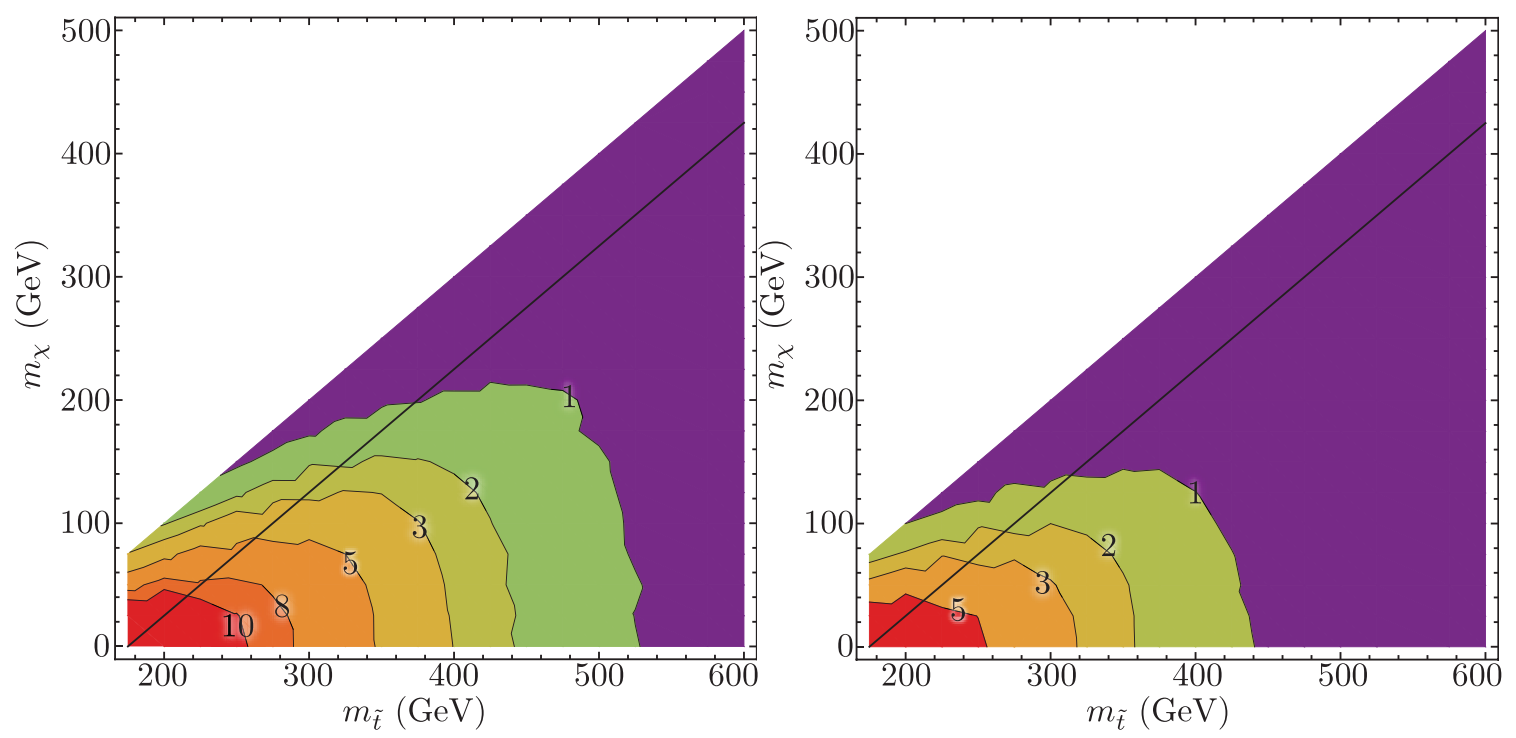

Figure 28: Expected number of standard deviations that the supersymmetric stop signal can be excluded by using 200 pseudo-experiments of $20 \mathrm{fb}^{-1}$, applying the $M_{T}^{W}$ shape analysis. In the left-hand plot the uncertainty on the background $\mathbb{E}_{T}$ shape are as shown in Table 10 and in the right-hand plot these errors have been inflated by a factor of 3 . 

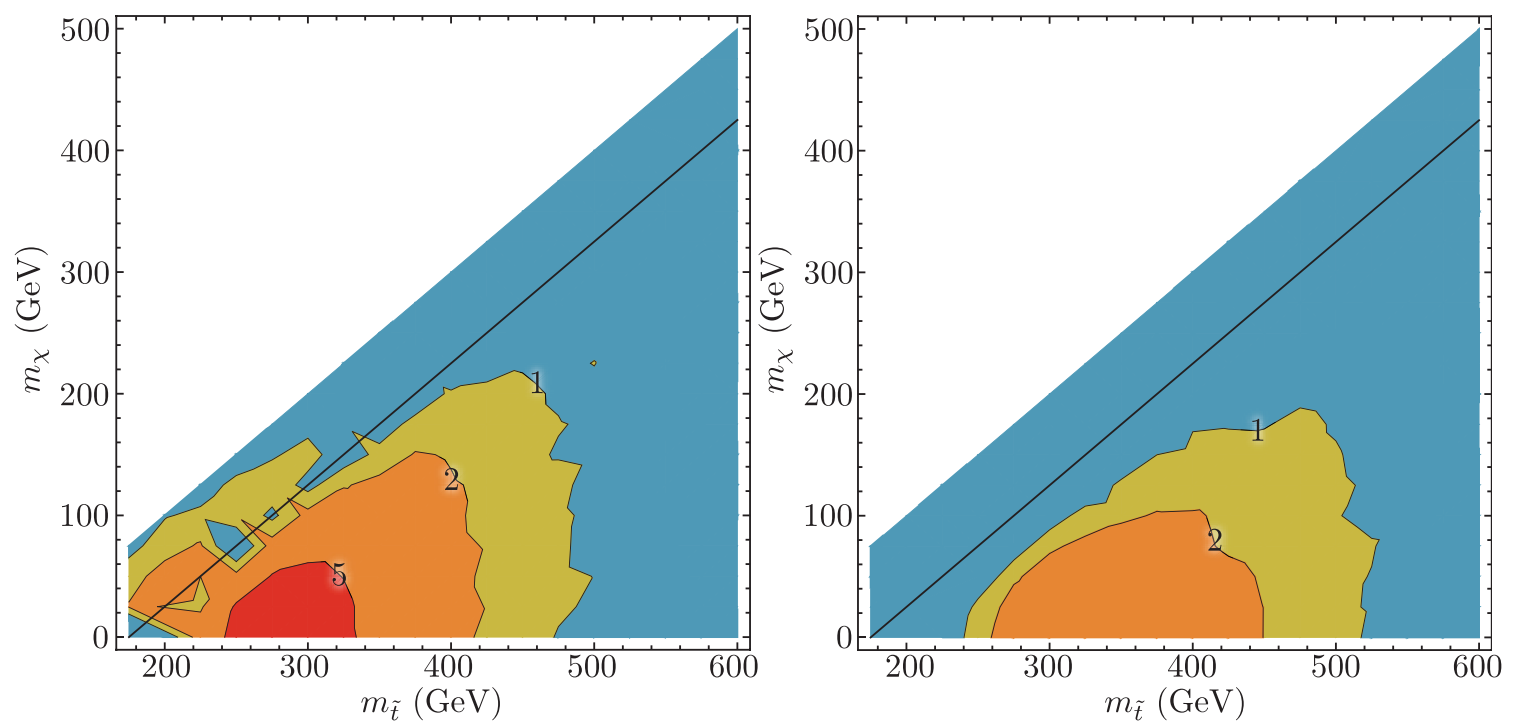

Figure 29: Left: $S / \sqrt{O}$ for $150-250 \mathrm{GeV}$ region of signal plus background $M_{T}^{W}$ distribution. Right: $S / \sqrt{O}$ for $250-400 \mathrm{GeV}$ region. These are computed with an unrealistic assumption of no systematics. 


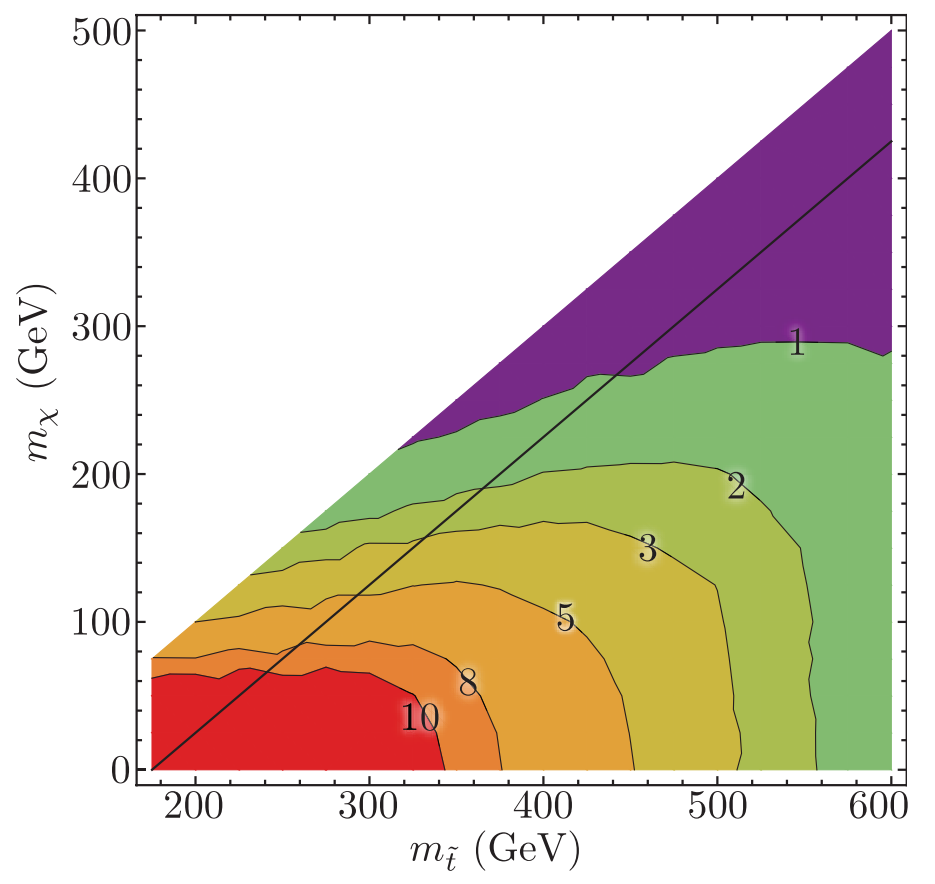

Figure 30: Expected sensitivity, in standard deviations, to SUSY stop signals using a combination of $\mathbb{E}_{T}$ and $M_{T}^{W}$ shape analyses, included all fitting errors in the maximum likelihood method. 


\section{Appendix A}

Below are formulae for $W^{+} W^{-}$production in the SM.

$$
\begin{gathered}
\sum|M|^{2}\left(\bar{d}_{L} d_{L} \rightarrow W^{+} W^{-}\right)=\left(\frac{e / s_{W}}{\sqrt{2}}\right)^{4} 4 E(\hat{s}, \hat{t}, \hat{u}) \\
+\left[-\frac{1}{3} e^{2}+\left(-\frac{1}{2}+\frac{s_{W}^{2}}{3}\right) \frac{e^{2}}{s_{W}^{2}} \frac{\hat{s}}{\hat{s}-M_{Z}^{2}}\right]^{2} 4 A(\hat{s}, \hat{t}, \hat{u}) \\
+2\left(\frac{e / s_{W}}{\sqrt{2}}\right)^{2}\left[-\frac{1}{3} e^{2}+\left(-\frac{1}{2}+\frac{s_{W}^{2}}{3}\right) \frac{e^{2}}{s_{W}^{2}} \frac{\hat{s}}{\hat{s}-M_{Z}^{2}}\right] 4 I(\hat{s}, \hat{t}, \hat{u})
\end{gathered}
$$

and

$$
\begin{gathered}
\sum|M|^{2}\left(\bar{u}_{L} u_{L} \rightarrow W^{+} W^{-}\right)=\left(\frac{e / s_{W}}{\sqrt{2}}\right)^{4} 4 E(\hat{s}, \hat{u}, \hat{t}) \\
+\left[\frac{2}{3} e^{2}+\left(\frac{1}{2}-\frac{2 s_{W}^{2}}{3}\right) \frac{e^{2}}{s_{W}^{2}} \frac{\hat{s}}{\hat{s}-M_{Z}^{2}}\right]^{2} 4 A(\hat{s}, \hat{u}, \hat{t}) \\
-2\left(\frac{e / s_{W}}{\sqrt{2}}\right)^{2}\left[\frac{2}{3} e^{2}+\left(\frac{1}{2}-\frac{2 s_{W}^{2}}{3}\right) \frac{e^{2}}{s_{W}^{2}} \frac{\hat{s}}{\hat{s}-M_{Z}^{2}}\right] 4 I(\hat{s}, \hat{u}, \hat{t})
\end{gathered}
$$

Note the interchange of $u \leftrightarrow t$ in the functions for the $u_{L}$ subprocess. The $\bar{u}_{R} u_{R}$ and $\bar{d}_{R} d_{R}$ processes only involve the $s$-channel in the SM,

$$
\sum|M|^{2}\left(\bar{d}_{R} d_{R} \rightarrow W^{+} W^{-}\right)=\left(-\frac{1}{3} e^{2}\right)^{2}\left(\frac{M_{Z}^{2}}{\hat{s}-M_{Z}^{2}}\right)^{2} 4 A(\hat{s}, \hat{t}, \hat{u})
$$

and

$$
\sum|M|^{2}\left(\bar{u}_{R} u_{R} \rightarrow W^{+} W^{-}\right)=\left(\frac{2}{3} e^{2}\right)^{2}\left(\frac{M_{Z}^{2}}{\hat{s}-M_{Z}^{2}}\right)^{2} 4 A(\hat{s}, \hat{u}, \hat{t})
$$


The formulas for the other vector boson pairs are [130]:

$$
\begin{gathered}
\sum|\mathcal{M}|^{2}(q \bar{q} \rightarrow Z \gamma)=2\left(e Q_{q}\right)^{2}\left(g_{L}^{q 2}+g_{R}^{q 2}\right)\left[\frac{\hat{s}^{2}+M_{Z}^{4}}{2 \hat{t} \hat{u}}-1\right], \\
\sum|M|^{2}(\bar{q} q \rightarrow Z Z)=\left(\left(g_{L}^{q}\right)^{4}+\left(g_{R}^{q}\right)^{4}\right)\left[\frac{\hat{t}}{\hat{u}}+\frac{\hat{u}}{\hat{t}}+\frac{4 M_{Z}^{2} \hat{s}}{\hat{u} \hat{t}}-M_{Z}^{4}\left(\frac{1}{\hat{t}^{2}}+\frac{1}{\hat{u}^{2}}\right)\right]
\end{gathered}
$$

and

$$
\begin{aligned}
\sum|\mathcal{M}|^{2}\left(q_{i} \bar{q}_{j} \rightarrow\right. & \left.Z W^{ \pm}\right)= \\
& \frac{2 e^{4}}{s_{W}^{2}}\left|V_{i j}\right|^{2}\left\{( \frac { 1 } { \hat { s } - M _ { W } ^ { 2 } } ) ^ { 2 } \left[\left(\frac{9-8 s_{W}^{2}}{4}\right)\left(\hat{u} \hat{t}-M_{W}^{2} M_{Z}^{2}\right)\right.\right. \\
+ & \left.\left(8 s_{W}^{2}-6\right) \hat{s}\left(M_{W}^{2}+M_{Z}^{2}\right)\right] \\
+ & {\left[\frac{\hat{u} \hat{t}-M_{W}^{2} M_{Z}^{2}-\hat{s}\left(M_{W}^{2}+M_{Z}^{2}\right)}{\hat{s}-M_{W}^{2}}\right]\left(\frac{g_{L}^{q_{j}}}{\hat{t}}-\frac{g_{L}^{q_{i}}}{\hat{u}}\right) } \\
+ & \frac{\hat{u} \hat{t}-M_{W}^{2} M_{Z}^{2}}{4\left(1-s_{W}^{2}\right)} \frac{1}{g_{Z}^{2}}\left(\frac{g_{L}^{q_{j}}}{\hat{t}^{2}}+\frac{g_{L}^{q_{2} 2}}{\hat{u}^{2}}\right) \\
+ & \left.\frac{\hat{s}\left(M_{W}^{2}+M_{Z}^{2}\right)}{2\left(1-s_{W}^{2}\right)} \frac{g_{L}^{q_{j}} g_{L}^{q_{i}}}{g_{Z}^{2} \hat{t} \hat{u}}\right\}
\end{aligned}
$$

where $g_{L, R}^{q}$ is the SM coupling between the quark pair and the $Z$ boson, and $e Q_{q}$ is the SM coupling between the quark pair to $\gamma$. The functions $A, I, E$ of Ref.[131] are

$$
\begin{aligned}
A(\hat{s}, \hat{t}, \hat{u}) & =\frac{1}{4}\left(\frac{\hat{u} \hat{t}}{M_{W}^{4}}-1\right)\left(1-4 \frac{M_{W}^{2}}{\hat{s}}+12 \frac{M_{W}^{4}}{\hat{s}^{2}}\right)+\frac{\hat{s}}{M_{W}^{2}}-4 \\
I(\hat{s}, \hat{t}, \hat{u}) & =\left[\frac{1}{4}\left(\frac{\hat{u} \hat{t}}{M_{W}^{4}}-1\right)\left(1-2 \frac{M_{W}^{2}}{\hat{s}}-\frac{4 M_{W}^{4}}{\hat{s} \hat{t}}\right)+\frac{\hat{s}}{M_{W}^{2}}-2+2 \frac{M_{W}^{2}}{\hat{t}}\right] \\
E(\hat{s}, \hat{t}, \hat{u}) & =\left[\frac{1}{4}\left(\frac{\hat{u} \hat{t}}{M_{W}^{4}}-1\right)\left(1+4 \frac{M_{W}^{4}}{\hat{t}^{2}}\right)+\frac{\hat{s}}{M_{W}^{2}}\right]
\end{aligned}
$$




\section{Bibliography}

[1] M. Baak, M. Goebel, J. Haller, A. Hoecker, D. Kennedy, et al. The Electroweak Fit of the Standard Model after the Discovery of a New Boson at the LHC. Eur.Phys.J., C72:2205, 2012.

[2] T. Aaltonen et al. Evidence for a Mass Dependent Forward-Backward Asymmetry in Top Quark Pair Production. Phys.Rev., D83:112003, 2011.

[3] T. Aaltonen et al. First Measurement of the $t$ anti-t Differential Cross Section d sigma $/ \mathrm{dM}(\mathrm{t}$ anti-t) in $\mathrm{p}$ anti-p Collisions at $\sqrt{s}=1.96 \mathrm{TeV}$. Phys.Rev.Lett., 102:222003, 2009.

[4] W. Beenakker, R. Hopker, and M. Spira. PROSPINO: A Program for the production of supersymmetric particles in next-to-leading order QCD. 1996.

[5] John Campbell and Keith Ellis. Monte Carlo for FeMtobarn processes.

[6] Makoto Kobayashi and Toshihide Maskawa. CP Violation in the Renormalizable Theory of Weak Interaction. Prog.Theor.Phys., 49:652-657, 1973.

[7] Tony M. Liss and P.L. Tipton. The discovery of the top quark. Sci.Am., 277N3:54-59, 1997.

[8] F. Abe et al. Observation of top quark production in $\bar{p} p$ collisions. Phys.Rev.Lett., 74:2626-2631, 1995. 
[9] S. Abachi et al. Observation of the top quark. Phys.Rev.Lett., 74:2632-2637, 1995.

[10] J. Beringer et al. Review of Particle Physics (RPP). Phys.Rev., D86:010001, 2012.

[11] Arnulf Quadt. Top quark physics at hadron colliders. Eur.Phys.J., C48:8351000, 2006.

[12] M. Baak, M. Goebel, J. Haller, A. Hoecker, and D. Kennedy. Gfitter.

[13] T. Aaltonen et al. Forward-Backward Asymmetry in Top Quark Production in $p \bar{p}$ Collisions at sqrts $=1.96 \mathrm{TeV}$. Phys.Rev.Lett., 101:202001, 2008.

[14] Victor Mukhamedovich Abazov et al. Forward-backward asymmetry in top quark-antiquark production. Phys.Rev., D84:112005, 2011.

[15] Glenn L. Strycker, Dan Amidei, Monica Tecchio, Thomas A. Schwarz, Robin Erbacher, and John Conway. Measurement of the forward-backward asymmetry in top pair production in $3.2 / \mathrm{fb}$ of ppbar collisions at sqrts $=1.96$ tev. 2009.

[16] Werner Bernreuther and Zong-Guo Si. Distributions and correlations for top quark pair production and decay at the Tevatron and LHC. Nucl.Phys., B837:90-121, 2010. 
[17] M.T. Bowen, S.D. Ellis, and D. Rainwater. Standard model top quark asymmetry at the Fermilab Tevatron. Phys.Rev., D73:014008, 2006.

[18] Johann H. Kuhn and German Rodrigo. Charge asymmetry in hadroproduction of heavy quarks. Phys.Rev.Lett., 81:49-52, 1998.

[19] V.M. Abazov et al. Combination of $t$ anti-t cross section measurements and constraints on the mass of the top quark and its decays into charged Higgs bosons. Phys.Rev., D80:071102, 2009.

[20] Nikolaos Kidonakis and Ramona Vogt. The Theoretical top quark cross section at the Tevatron and the LHC. Phys.Rev., D78:074005, 2008.

[21] Sven Moch and Peter Uwer. Theoretical status and prospects for top-quark pair production at hadron colliders. Phys.Rev., D78:034003, 2008.

[22] Matteo Cacciari, Stefano Frixione, Michelangelo L. Mangano, Paolo Nason, and Giovanni Ridolfi. Updated predictions for the total production cross sections of top and of heavier quark pairs at the Tevatron and at the LHC. JHEP, 0809:127, 2008.

[23] S. Moch and P. Uwer. Heavy-quark pair production at two loops in QCD. Nucl.Phys.Proc.Suppl., 183:75-80, 2008.

[24] Oscar Antunano, Johann H. Kuhn, and German Rodrigo. Top quarks, axigluons and charge asymmetries at hadron colliders. Phys.Rev., D77:014003, 2008. 
[25] Paul H. Frampton, Jing Shu, and Kai Wang. Axigluon as Possible Explanation for p anti-p to t anti-t Forward-Backward Asymmetry. Phys.Lett., B683:294$297,2010$.

[26] Jing Shu, Tim M.P. Tait, and Kai Wang. Explorations of the Top Quark Forward-Backward Asymmetry at the Tevatron. Phys.Rev., D81:034012, 2010.

[27] Sunghoon Jung, Hitoshi Murayama, Aaron Pierce, and James D. Wells. Top quark forward-backward asymmetry from new t-channel physics. Phys.Rev., D81:015004, 2010.

[28] Kingman Cheung, Wai-Yee Keung, and Tzu-Chiang Yuan. Top Quark Forward-Backward Asymmetry. Phys.Lett., B682:287-290, 2009.

[29] Junjie Cao, Zhaoxia Heng, Lei Wu, and Jin Min Yang. Top quark forwardbackward asymmetry at the Tevatron: A Comparative study in different new physics models. Phys.Rev., D81:014016, 2010.

[30] Dong-Won Jung, P. Ko, Jae Sik Lee, and Soo-hyeon Nam. Model independent analysis of the forward-backward asymmetry of top quark production at the Tevatron. Phys.Lett., B691:238-242, 2010.

[31] Ilja Dorsner, Svjetlana Fajfer, Jernej F. Kamenik, and Nejc Kosnik. Light colored scalars from grand unification and the forward-backward asymmetry in t t-bar production. Phys.Rev., D81:055009, 2010. 
[32] Paola Ferrario and German Rodrigo. Heavy colored resonances in t t-bar + jet at the LHC. JHEP, 1002:051, 2010.

[33] Abdesslam Arhrib, Rachid Benbrik, and Chuan-Hung Chen. Forwardbackward asymmetry of top quark in diquark models. Phys.Rev., D82:034034, 2010.

[34] Qing-Hong Cao, David McKeen, Jonathan L. Rosner, Gabe Shaughnessy, and Carlos E.M. Wagner. Forward-Backward Asymmetry of Top Quark Pair Production. Phys.Rev., D81:114004, 2010.

[35] Vernon D. Barger and R.J.N. Phillips. COLLIDER PHYSICS. 1987.

[36] Johan Alwall, Pavel Demin, Simon de Visscher, Rikkert Frederix, Michel Herquet, et al. MadGraph/MadEvent v4: The New Web Generation. JHEP, 0709:028, 2007.

[37] Pavel M. Nadolsky, Hung-Liang Lai, Qing-Hong Cao, Joey Huston, Jon Pumplin, et al. Implications of CTEQ global analysis for collider observables. Phys.Rev., D78:013004, 2008.

[38] Kirill Melnikov and Markus Schulze. NLO QCD corrections to top quark pair production and decay at hadron colliders. JHEP, 0908:049, 2009.

[39] Claude Amsler et al. Review of Particle Physics. Phys.Lett., B667:1-1340, 2008. 
[40] Tevatron Electroweak Working Group. Combination of CDF and D0 Results on the Mass of the Top Quark. 2009.

[41] T. Aaltonen et al. A Search for high-mass resonances decaying to dimuons at CDF. Phys.Rev.Lett., 102:091805, 2009.

[42] Martin Bauer, Florian Goertz, Ulrich Haisch, Torsten Pfoh, and Susanne Westhoff. Top-Quark Forward-Backward Asymmetry in Randall-Sundrum Models Beyond the Leading Order. JHEP, 1011:039, 2010.

[43] Chuan-Hung Chen, G. Cvetic, and C.S. Kim. Forward-backward asymmetry of top quark in unparticle physics. Phys.Lett., B694:393-397, 2011.

[44] Dong-Won Jung, P. Ko, and Jae Sik Lee. Longitudinal top polarization as a probe of a possible origin of forward-backward asymmetry of the top quark at the Tevatron. Phys.Lett., B701:248-254, 2011.

[45] Junjie Cao, Lin Wang, Lei Wu, and Jin Min Yang. Top quark forward-backward asymmetry, FCNC decays and like-sign pair production as a joint probe of new physics. Phys.Rev., D84:074001, 2011.

[46] Yang Bai, JoAnne L. Hewett, Jared Kaplan, and Thomas G. Rizzo. LHC Predictions from a Tevatron Anomaly in the Top Quark Forward-Backward Asymmetry. JHEP, 1103:003, 2011.

[47] Edmond L. Berger, Qing-Hong Cao, Chuan-Ren Chen, Chong Sheng Li, and 
Hao Zhang. Top Quark Forward-Backward Asymmetry and Same-Sign Top Quark Pairs. Phys.Rev.Lett., 106:201801, 2011.

[48] Cedric Delaunay, Oram Gedalia, Seung J. Lee, Gilad Perez, and Eduardo Ponton. Extraordinary Phenomenology from Warped Flavor Triviality. Phys.Lett., B703:486-490, 2011.

[49] Leandro G. Almeida, George F. Sterman, and Werner Vogelsang. Threshold Resummation for the Top Quark Charge Asymmetry. Phys.Rev., D78:014008, 2008.

[50] Kingman Cheung and Tzu-Chiang Yuan. Top Quark Forward-Backward Asymmetry in the Large Invariant Mass Region. Phys.Rev., D83:074006, 2011.

[51] Vernon Barger, Wai-Yee Keung, and Chiu-Tien Yu. Asymmetric LeftRight Model and the Top Pair Forward-Backward Asymmetry. Phys.Rev., D81:113009, 2010.

[52] T. Aaltonen et al. Search for High Mass Resonances Decaying to Muon Pairs in $\sqrt{s}=1.96 \mathrm{TeV} p \bar{p}$ Collisions. Phys.Rev.Lett., 106:121801, 2011.

[53] T. Aaltonen et al. Search for new particles decaying into dijets in protonantiproton collisions at $\sqrt{s}=1.96$ TeV. Phys.Rev., D79:112002, 2009.

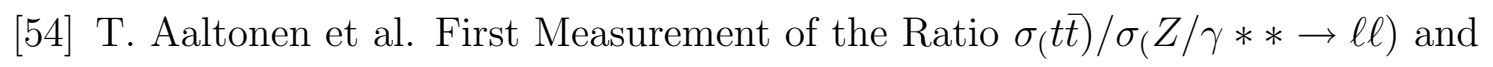
Precise Extraction of the $t \bar{t}$ Cross Section. Phys.Rev.Lett., 105:012001, 2010. 
[55] Vernon Barger, Tao Han, and Devin G.E. Walker. Top Quark Pairs at High Invariant Mass: A Model-Independent Discriminator of New Physics at the LHC. Phys.Rev.Lett., 100:031801, 2008.

[56] Martin Schmaltz and Christian Spethmann. Two Simple W' Models for the Early LHC. JHEP, 1107:046, 2011.

[57] Shrihari Gopalakrishna, Tao Han, Ian Lewis, Zong-guo Si, and Yu-Feng Zhou. Chiral Couplings of 'W' and Top Quark Polarization at the LHC. Phys.Rev., D82:115020, 2010 .

[58] Stephen Godfrey and Ken Moats. Exploring Higgs Triplet Models via Vector Boson Scattering at the LHC. Phys.Rev., D81:075026, 2010.

[59] Jessie Shelton and Kathryn M. Zurek. Maximal flavor violation from new right-handed gauge bosons. Phys.Rev., D83:091701, 2011.

[60] D.J. Gross and Frank Wilczek. Ultraviolet Behavior of Nonabelian Gauge Theories. Phys.Rev.Lett., 30:1343-1346, 1973.

[61] Cumrun Vafa and Edward Witten. Parity Conservation in QCD. Phys.Rev.Lett., 53:535, 1984.

[62] R.D. Peccei and Helen R. Quinn. CP Conservation in the Presence of Instantons. Phys.Rev.Lett., 38:1440-1443, 1977. 
[63] Dmitri Kharzeev, R.D. Pisarski, and Michel H.G. Tytgat. Possibility of spontaneous parity violation in hot QCD. Phys.Rev.Lett., 81:512-515, 1998.

[64] B.I. Abelev et al. Observation of charge-dependent azimuthal correlations and possible local strong parity violation in heavy ion collisions. Phys.Rev., C81:054908, 2010.

[65] Gustavo Marques Tavares and Martin Schmaltz. Explaining the t-tbar asymmetry with a light axigluon. Phys.Rev., D84:054008, 2011.

[66] Ulrich Haisch and Susanne Westhoff. Massive Color-Octet Bosons: Bounds on Effects in Top-Quark Pair Production. JHEP, 1108:088, 2011.

[67] Moira I. Gresham, Ian-Woo Kim, and Kathryn M. Zurek. Tevatron Top $A_{F B}$ Versus LHC Top Physics. Phys.Rev., D85:014022, 2012.

[68] J.A. Aguilar-Saavedra and M. Perez-Victoria. Simple models for the top asymmetry: Constraints and predictions. JHEP, 1109:097, 2011.

[69] Paul H. Frampton and Sheldon L. Glashow. Chiral Color: An Alternative to the Standard Model. Phys.Lett., B190:157, 1987.

[70] Gordon L. Kane, G.A. Ladinsky, and C.P. Yuan. Using the Top Quark for Testing Standard Model Polarization and CP Predictions. Phys.Rev., D45:124141, 1992. 
[71] Debajyoti Choudhury, Rohini M. Godbole, Saurabh D. Rindani, and Pratishruti Saha. Top polarization, forward-backward asymmetry and new physics. Phys.Rev., D84:014023, 2011.

[72] Rohini M. Godbole, Kumar Rao, Saurabh D. Rindani, and Ritesh K. Singh. On measurement of top polarization as a probe of $t \bar{t}$ production mechanisms at the LHC. JHEP, 1011:144, 2010.

[73] David Krohn, Tao Liu, Jessie Shelton, and Lian-Tao Wang. A Polarized View of the Top Asymmetry. Phys.Rev., D84:074034, 2011.

[74] Ryuichiro Kitano and Yasunori Nomura. Supersymmetry, Naturalness, and Signatures at the Lhc. Phys. Rev., D73:095004, 2006.

[75] Masaki Asano, Hyung Do Kim, Ryuichiro Kitano, and Yasuhiro Shimizu. Natural Supersymmetry at the Lhc. JHEP, 12:019, 2010.

[76] Serguei Chatrchyan et al. Search for Supersymmetry at the Lhc in Events with Jets and Missing Transverse Energy. Phys. Rev. Lett., 107:221804, 2011.

[77] Georges Aad et al. Search for squarks and gluinos using final states with jets and missing transverse momentum with the ATLAS detector in $\operatorname{sqrt}(\mathrm{s})=7$ TeV proton-proton collisions. Phys.Lett., B710:67-85, 2012.

[78] S. Chatrchyan et al. Search for supersymmetry in final states with a single lepton, b-jets, and missing transverse energy in proton-proton collisions at $\mathrm{s}=$ 7 tev. CMS-PAS-SUS-11-028, 2012. 
[79] Georges Aad et al. Search for scalar top quark pair production in natural gauge mediated supersymmetry models with the ATLAS detector in pp collisions at $\operatorname{sqrt}(\mathrm{s})=7 \mathrm{TeV} .2012$.

[80] A. Djouadi, W. Hollik, and C. Junger. QCD Corrections to the Top Decay Mode T —i Supersymmetric T Chi0. Phys. Rev., D54:5629-5635, 1996.

[81] S. Kraml, H. Eberl, A. Bartl, W. Majerotto, and W. Porod. Susy-QCD Corrections to Scalar Quark Decays into Charginos and Neutralinos. Phys. Lett., B386:175-182, 1996.

[82] W. Beenakker, R. Hopker, T. Plehn, and P. M. Zerwas. Stop Decays in SusyQCD. Z. Phys., C75:349-356, 1997.

[83] CDF Collaboration. CDF Note 9834, 2009.

[84] Manuel Drees, M. Hanussek, and Jong Soo Kim. Light Stop Searches at the Lhc with Monojet Events. 2012.

[85] T. Aaltonen et al. Search for Scalar Top Quark Production in $p \bar{p}$ Collisions at $\sqrt{s}=1.96 \mathrm{TeV} .2012$.

[86] Victor Mukhamedovich Abazov et al. Search for pair production of the scalar top quark in the electron+muon final state. Phys.Lett., B696:321-327, 2011.

[87] M. Carena, A. Freitas, and C.E.M. Wagner. Light Stop Searches at the LHC 
in Events with One Hard Photon or Jet and Missing Energy. JHEP, 0810:109, 2008.

[88] Bin He, Tong Li, and Qaisar Shafi. Impact of LHC Searches on Light Top Squark. 2011.

[89] Xiao-Jun Bi, Qi-Shu Yan, and Peng-Fei Yin. Probing Light Stop Pairs at the LHC. Phys.Rev., D85:035005, 2012.

[90] Patrick Meade and Matthew Reece. Top Partners at the Lhc: Spin and Mass Measurement. Phys. Rev., D74:015010, 2006.

[91] Tao Han, Rakhi Mahbubani, Devin G. E. Walker, and Lian-Tao Wang. Top Quark Pair Plus Large Missing Energy at the Lhc. JHEP, 05:117, 2009.

[92] Yang Bai, Hsin-Chia Cheng, Jason Gallicchio, and Jiayin Gu. Stop the Top Background of the Stop Search. 2012.

[93] Tilman Plehn, Michael Spannowsky, Michihisa Takeuchi, and Dirk Zerwas. Stop Reconstruction with Tagged Tops. JHEP, 10:078, 2010.

[94] Tilman Plehn, Michael Spannowsky, and Michihisa Takeuchi. Boosted Semileptonic Tops in Stop Decays. JHEP, 05:135, 2011.

[95] Tilman Plehn, Michael Spannowsky, and Michihisa Takeuchi. Stop searches in 2012. 2012. 
[96] Yevgeny Kats and David Shih. Light Stop Nlsps at the Tevatron and Lhc. JHEP, 08:049, 2011.

[97] Yevgeny Kats, Patrick Meade, Matthew Reece, and David Shih. The Status of Gmsb After 1/Fb at the Lhc. 2011.

[98] ATLAS Collaboration. ATLAS-CONF-2011-100.

[99] Georges Aad et al. Search for New Phenomena in Ttbar Events with Large Missing Transverse Momentum in Proton-Proton Collisions at $\operatorname{Sqrt}(\mathrm{S})=7 \mathrm{TeV}$ with the Atlas Detector. Phys. Rev. Lett., 108:041805, 2012.

[100] V. M. Abazov et al. Search for the Lightest Scalar Top Quark in Events with Two Leptons in P Anti-P Collisions at $S^{(1 / 2)}=1.96-\mathrm{TeV}$. Phys. Lett., B675:289-296, 2009.

[101] T. Aaltonen et al. Search for Pair Production of Supersymmetric Top Quarks in Dilepton Events from $p \bar{p}$ Collisions at $\sqrt{s}=1.96 \mathrm{TeV}$. Phys. Rev. Lett., 104:251801, 2010.

[102] T. Aaltonen et al. Search for Production of Heavy Particles Decaying to Top Quarks and Invisible Particles in $p \bar{p}$ Collisions at $\sqrt{s}=1.96 \mathrm{TeV}$. Phys. Rev. Lett., 106:191801, 2011.

[103] T. Aaltonen et al. Search for New $T^{\prime}$ Particles in Final States with Large Jet Multiplicities and Missing Transverse Energy in PPbar Collisions at $\sqrt{s}=1.96$ TeV. Phys. Rev. Lett., 107:191803, 2011. 
[104] Victor Mukhamedovich Abazov et al. Search for pair production of the scalar top quark in muon+tau final states. 2012.

[105] Zhenyu Han, Andrey Katz, David Krohn, and Matthew Reece. (Light) Stop Signs. 2012.

[106] David E. Kaplan, Keith Rehermann, and Daniel Stolarski. Searching for Direct Stop Production in Hadronic Top Data at the LHC. 2012.

[107] Csaba Csaki, Lisa Randall, and John Terning. Light Stops from Seiberg Duality. 2012 .

[108] Georges Aad et al. Hunt for new phenomena using large jet multiplicities and missing transverse momentum with ATLAS in $\mathrm{L}=4.7 \mathrm{fb}^{-1}$ of $\operatorname{sqrt}(\mathrm{s})=7 \mathrm{TeV}$ proton-proton collisions. ATLAS-CONF-2012-037, 2012.

[109] S. Chatrchyan et al. Inclusive search for squarks and gluinos in $p p$ collisions at $\sqrt{s}=7$ TeV. Phys. Rev. D, 85:012004, Jan 2012.

[110] S. Chatrchyan et al. Search for supersymmetry with the razor variables at CMS. CMS-PAS-SUS-12-005, 2012.

[111] S. Chatrchyan et al. Search for Pair Production of Third-Generation Scalar Leptoquarks Using Events Produced in pp Collisions at sqrt(s) $=7$ TeV. CMSPAS-EXO-11-030, 2012. 
[112] Christopher Rogan. Kinematical variables towards new dynamics at the LHC. 2010.

[113] Daniele Alves et al. Simplified Models for LHC New Physics Searches. 2011.

[114] CMS Collaboration. Missing transverse energy performance of the CMS detector. oai:cds.cern.ch:1361973, Jun 2011.

[115] Georges Aad et al. Performance of Missing Transverse Momentum Reconstruction in Proton-Proton Collisions at 7 TeV with ATLAS. Eur.Phys.J., C72:1844, 2012.

[116] S. Chatrchyan et al. Search for supersymmetry with the razor variables at cms. 2011. CMS-PAS-SUS-11-008.

[117] Tevatron Electroweak Working Group. 2012 Update of the Combination of CDF and D0 Results for the Mass of the W Boson. 2012.

[118] Johan Alwall, Michel Herquet, Fabio Maltoni, Olivier Mattelaer, and Tim Stelzer. Madgraph 5 : Going beyond. 062011.

[119] Torbjorn Sjostrand, Stephen Mrenna, and Peter Skands. Pythia 6.4 physics and manual. 2006.

[120] John Conway et al. PGS-Pretty Good Simulation, 2009. http://physics. ucdavis.edu/ conway/research/software/pgs/pgs4-general.htm. 
[121] Jay Hubisz, Joseph Lykken, Maurizio Pierini, and Maria Spiropulu. Missing energy look-alikes with $100 \mathrm{pb}^{-1}$ at the LHC. Phys.Rev., D78:075008, 2008.

[122] Maurizio Pierini. Experiment: Presentation of scientific results. Chicago 2012 workshop on LHC physics, 2012.

[123] Wouter Verkerke and David P. Kirkby. The RooFit toolkit for data modeling. eConf, C0303241:MOLT007, 2003.

[124] CMS Collaboration. CMS PAS BTV-11-001.

[125] Georges Aad et al. Measurement of multi-jet cross sections in proton-proton collisions at a 7 TeV center-of-mass energy. Eur.Phys.J., C71:1763, 2011.

[126] Michelangelo L. Mangano, Mauro Moretti, Fulvio Piccinini, Roberto Pittau, and Antonio D. Polosa. ALPGEN, a generator for hard multiparton processes in hadronic collisions. JHEP, 0307:001, 2003.

[127] Glen Cowan, Kyle Cranmer, Eilam Gross, and Ofer Vitells. Asymptotic formulae for likelihood-based tests of new physics. Eur.Phys.J., C71:1554, 2011.

[128] Thomas Junk. Confidence level computation for combining searches with small statistics. Nucl.Instrum.Meth., A434:435-443, 1999.

[129] Alexander L. Read. Presentation of search results: The CL(s) technique. J.Phys.G, G28:2693-2704, 2002. 
[130] E. Eichten, I. Hinchliffe, Kenneth D. Lane, and C. Quigg. Super Collider Physics. Rev.Mod.Phys., 56:579-707, 1984.

[131] R.W. Brown and K.O. Mikaelian. W+ W- and Z0 Z0 Pair Production in e+ e-, p p, p anti-p Colliding Beams. Phys.Rev., D19:922, 1979. 